\title{
New Martingale Inequalities and Applications to Fourier Analysis
}

\author{
Guangheng Xie, Ferenc Weisz, Dachun Yang* and Yong Jiao
}

\begin{abstract}
Let $(\Omega, \mathcal{F}, \mathbb{P})$ be a probability space and $\varphi: \Omega \times[0, \infty) \rightarrow[0, \infty)$ be a MusielakOrlicz function. In this article, the authors prove that the Doob maximal operator is bounded on the Musielak-Orlicz space $L^{\varphi}(\Omega)$. Using this and extrapolation method, the authors then establish a Fefferman-Stein vector-valued Doob maximal inequality on $L^{\varphi}(\Omega)$. As applications, the authors obtain the dual version of the Doob maximal inequality and the Stein inequality for $L^{\varphi}(\Omega)$, which are new even in weighted Orlicz spaces. The authors then establish the atomic characterizations of martingale Musielak-Orlicz Hardy spaces $H_{\varphi}^{s}(\Omega)$, $P_{\varphi}(\Omega), Q_{\varphi}(\Omega), H_{\varphi}^{S}(\Omega)$ and $H_{\varphi}^{M}(\Omega)$. From these atomic characterizations, the authors further deduce some martingale inequalities between different martingale Musielak-Orlicz Hardy spaces, which essentially improve the corresponding results in Orlicz space case and are also new even in weighted Orlicz spaces. By establishing the Davis decomposition on $H_{\varphi}^{S}(\Omega)$ and $H_{\varphi}^{M}(\Omega)$, the authors obtain the Burkholder-Davis-Gundy inequality associated with Musielak-Orlicz functions. Finally, using the previous martingale inequalities, the authors prove that the maximal Fejér operator is bounded from $H_{\varphi}[0,1)$ to $L^{\varphi}[0,1)$, which further implies some convergence results of the Fejér means; these results are new even for the weighted Hardy spaces.
\end{abstract}

\section{Introduction}

As is well known, the martingale theory has been well developed since Doob [19]. Using a maximal inequality, which is now called the Doob maximal inequality, Doob [19] proved the basic almost sure convergence properties of the martingales. This topic have been studied particularly intensively by Burkholder. As for probability theory, specially for martingale theory, the references greatly influence us are $[19,23,32,64,77]$ and the articles $[9,10,11]$. Notice that the martingale theory has an extensive application in dyadic harmonic analysis, we refer the reader to the monographes $[68,77,78]$.

Let $\mathbb{Z}_{+}:=\{0\} \cup \mathbb{N}:=\{0,1, \ldots\},(\Omega, \mathcal{F}, \mathbb{P})$ be a probability space, $\left\{\mathcal{F}_{n}\right\}_{n \in \mathbb{Z}_{+}}$an increasing sequence of sub- $\sigma$-algebras of $\mathcal{F}$ and $\left\{\mathbb{E}_{n}\right\}_{n \in \mathbb{Z}_{+}}$the associated conditional expectations. For any $n \in \mathbb{Z}_{+}$and

2010 Mathematics Subject Classification. Primary 60G42; Secondary 60G46, 42B25, 42B35, 46E30.

Key words and phrases. Probability space, Musielak-Orlicz space, martingale Musielak-Orlicz Hardy space, quadratic variation, atom, Doob maximal operator, Fejér operator, Burkholder-Davis-Gundy inequality, weight.

This project is supported by the National Natural Science Foundation of China (Grant Nos. 11571039, 11726621, 11761131002, 11471337 and 11722114) and the Hungarian Scientific Research Funds (OTKA) No K115804.

*Corresponding author/September 27, 2018/Final version. 
measurable function $f$, the Doob maximal operators $M_{n}(f)$ and $M(f)$ are defined, respectively, by setting

$$
M_{n}(f):=\sup _{0 \leq i \leq n}\left|\mathbb{E}_{i}(f)\right| \text { and } M(f):=\sup _{n \in \mathbb{Z}_{+}}\left|\mathbb{E}_{n}(f)\right| .
$$

Let $p \in(0, \infty)$ and $w$ be a weight, the weighted Lebesgue space $L^{p}(\Omega, w d \mathbb{P})$ is defined to be the set of all measurable functions $f$ on $\Omega$ such that

$$
\|f\|_{L^{p}(\Omega, w d \mathbb{P})}:=\left[\int_{\Omega}|f(x)|^{p} w(x) d \mathbb{P}\right]^{\frac{1}{p}}<\infty .
$$

In 1977, Izumisawa and Kazamaki [33] obtained that $w \in A_{p_{0}}(\Omega)$ (see Section 2 below for its definition) for some $p_{0} \in(1, \infty)$ implies that the Doob maximal operator $M$ is bounded on $L^{p}(\Omega, w d \mathbb{P})$ for any $p \in\left(p_{0}, \infty\right)$. After this, Long [50, Theorem 6.6.3] improved their result by proving that $w \in A_{p}(\Omega)$ with $p \in(1, \infty)$ if and only if the Doob maximal operator $M$ is bounded on $L^{p}(\Omega, w d \mathbb{P})$. Recently, the sharp weighted Doob maximal inequalities have been studied by Osękowski [59, 60].

On another hand, the classical Fefferman-Stein vector-valued inequality was proved by Fefferman and Stein in their celebrated paper [20]. Later, Andersen and John [1, Theorem 3.1] established the weighted version of the vector-valued inequality for the Hardy-Littlewood maximal operator. For the Doob maximal operator, Jiao et al. [37, Theorem 6.1] proved the FeffermanStein theorem in rearrangement invariant spaces, Hytönen et al. [32, Theorem 3.2.7] gave a version in the Banach-valued setting.

The well-known Burkholder-Davis-Gundy inequality was proved by Burkholder et al. in their excellent article [10, Theorem 1.1], and is read as follows: Let $\Phi:[0, \infty) \rightarrow[0, \infty)$ be an Orlicz function. If $\Phi$ is convex and there exists a positive constant $C$ such that, for any $\lambda \in(0, \infty)$, $\Phi(2 \lambda) \leq C \Phi(\lambda)$, then, for any martingale $f:=\left(f_{n}\right)_{n \in \mathbb{Z}_{+}}$,

$$
\int_{\Omega} \Phi(S(f)) d \mathbb{P} \sim \int_{\Omega} \Phi(M(f)) d \mathbb{P}
$$

where $S(f):=\left(\sum_{n=1}^{\infty}\left|f_{n}-f_{n-1}\right|^{2}\right)^{1 / 2}$ and the equivalent positive constants are independent of $f$. After their outstanding work, Bonami and Lépingle [7, Theorem 1] proved the weighted version of (1.2). Then Johnson and Schechtman [41, Theorem 3] extended (1.2) to the setting of rearrangement invariant function spaces.

Moreover, more martingale inequalities were recently studied by Osękowski [61, 62], Kikuchi $[44,45,46]$ and Ho $[31,30]$. Especially, Bañuelos and Osȩkowski studied the weighted martingale inequalities in $[3,4]$. We refer the reader to recent monographs $[32,63,64]$ for more discussions on martingale inequalities. On another hand, various martingale Hardy spaces were considered in many articles, for instance, martingale Hardy spaces, martingale Lorentz Hardy spaces and martingale variable Hardy spaces were studied by Weisz [76, 77, 75] and Jiao et al. [49, 40, 39, 35, 38]. Moreover, martingale Lorentz-Karamata Hardy spaces and multi-parameter martingale Hardy spaces were investigated by Ho [29], Jiao et al. [36] and Weisz [75]. Variable martingale Hardy spaces, martingale Morrey Hardy spaces, martingale BLO spaces, martingale Besov spaces and Tribel-Lizorkin spaces were studied by Nakai et al. [56, 55, 57, 52, 66]. Martingale MusielakOrlicz Hardy spaces were dealt with by Xie at al. in [79] which mainly concern Musielak-Orlicz 
Hardy spaces determined by Musielak-Orlicz functions of uniformly upper type $p=1$ only (see Definition 2.9 below for its definition).

The theory of martingales has an extensive application in dyadic Fourier analysis; see, for example, the monographes by Schipp et al. [68] and Golubov et al. [25]. In particular, WalshFourier series were investigated for example in the monographes [68, 25] and the article [78]. Besides the partial sums $\left(s_{k} f\right)_{k \in \mathbb{N}}$ (see Section 6 below for its definition) of the Walsh-Fourier series of a martingale $f$, many articles (see, for example, $[40,74]$ ) also considered the Fejér means defined by setting

$$
\sigma_{n} f:=\frac{1}{n} \sum_{k=1}^{n} s_{k} f, \quad \forall n \in \mathbb{N} .
$$

It is known that, to obtain some convergence results for the Fejér means, one needs to investigate the maximal Fejér operator $\sigma_{*}$ defined by setting, for any martingale $f$,

$$
\sigma_{*} f:=\sup _{n \in \mathbb{N}}\left|\sigma_{n} f\right|
$$

In $[74,78]$, using dyadic martingale theory, Weisz proved that the maximal operator $\sigma_{*}$ is bounded from $H_{p}[0,1)$ to $L^{p}[0,1)$ for any given $p \in\left(\frac{1}{2}, \infty\right)$. Very recently, Jiao et al. [40, Theorem 7.15] investigated the boundedness of $\sigma_{*}$ from variable Hardy spaces to variable Lebesgue spaces.

Recall that a function $\varphi: \Omega \times[0, \infty) \rightarrow[0, \infty)$ is called a Musielak-Orlicz function if for any $x \in \Omega$, the function $\varphi(x, \cdot):[0, \infty) \rightarrow[0, \infty)$ is an Orlicz function, namely, $\varphi(x, \cdot)$ is nondecreasing, $\varphi(x, 0)=0$ and $\lim _{t \rightarrow \infty} \varphi(x, t)=\infty$, and the function $\varphi(\cdot, t)$ is a measurable function for any given $t \in[0, \infty)$. The Musielak-Orlicz space $L^{\varphi}(\Omega)$ is defined to be the set of all measurable functions $f$ with finite Luxemburg (also called as the Luxemburg-Nakano) norms $\|f\|_{L^{\varphi}(\Omega)}$ defined by setting

$$
\|f\|_{L^{\varphi}(\Omega)}:=\inf \left\{\lambda \in(0, \infty): \int_{\Omega} \varphi(x,|f(x)| / \lambda) d \mathbb{P}(x) \leq 1\right\} .
$$

It was originated by Nakano [58] and developed by Musielak and Orlicz [53, 54].

Observe that Musielak-Orlicz spaces are the natural generalization of many important spaces. For example, let $p \in(0, \infty), w$ be a weight, $\Phi$ an Orlicz function and $p(\cdot): \mathbb{R}^{d} \rightarrow[1, \infty]$ a measurable function. If $\varphi(x, t):=t^{p}, w(x) t^{p}, \Phi(t)$ or $t^{p(x)}$ for any $x \in \mathbb{R}^{d}$ and $t \in(0, \infty)$, then $L^{\varphi}\left(\mathbb{R}^{d}\right)$ become the classical Lebesgue spaces, the weighted Lebesgue spaces, Orlicz spaces (see, for example, [65]) or the variable Lebesgue spaces (see, for example, [14]), respectively. Moreover, if $\varphi(x, t):=t^{p}+w(x) t^{q}$ for any $x \in \mathbb{R}^{d}$ and $t \in(0, \infty), 1<p \leq q<\infty$, we obtain the double phase functionals (see, for example, [5, 13]). For more examples, see Example 2.16 below. The Musielak-Orlicz spaces not only have their own interest, but they are also very useful in partial differential equations [2, 5, 28, 26], in calculus of variations [13], in image restoration $[27,43]$ and in fluid dynamics $[73,51]$.

Furthermore, Musielak-Orlicz Hardy spaces on $\mathbb{R}^{d}$ are fruitful in dealing with many problems of analysis; see, for example, [12, 48, 34, 80]. Especially, they naturally appear in the endpoint estimates for the div-curl lemma and the commutators of Caldrón-Zygmund operators (see $[6,8,80]$ ). See also the monograph [80] for a detailed and complete survey of the recent progress related to the real-variable theory and its applications of Musielak-Orlicz Hardy spaces. 
Although the theory of Musielak-Orlicz Hardy spaces has rapidly been developed in recent years, the corresponding martingale theory associated with Musielak-Orlicz functions has not yet been developed well.

In this article, we first obtain the boundedness of the Doob maximal operator on $L^{\varphi}(\Omega)$. Using this and extrapolation method, we prove the weighted Fefferman-Stein vector-valued Doob maximal inequality and a Fefferman-Stein vector-valued inequality on $L^{\varphi}(\Omega)$. As applications, we obtain the dual version of the Doob maximal inequality, the weak type maximal inequality and the Stein inequality on $L^{\varphi}(\Omega)$, which are new even in weighed Orlicz spaces. Then we establish the atomic characterizations of martingale Musielak-Orlicz Hardy spaces $H_{\varphi}^{s}(\Omega), P_{\varphi}(\Omega), Q_{\varphi}(\Omega)$, $H_{\varphi}^{S}(\Omega)$ and $H_{\varphi}^{M}(\Omega)$, which is totally different from the classical martingale Hardy spaces (see, for example, [76]) and the classical martingale Orlicz Hardy spaces (see, for example, [52]). Using these atomic characterizations, we explore the relationship among these martingale MusielakOrlicz Hardy spaces. Our theorems improve the Orlicz case [52, Theorem 2.5 and Corollary 2.6] of Miyamoto et al. Moreover, our martingale inequalities partially improve Kazamaki [42, Theorem 1]. Since Musielak-Orlicz functions unify the weight and the Orlicz function, and MusielakOrlicz spaces are not rearrangement invariant, it is natural to ask whether or not (1.2) still holds true on $L^{\varphi}(\Omega)$. We give an affirmative answer to this question. Indeed, by establishing the Davis decomposition of $H_{\varphi}^{S}(\Omega)$ and $H_{\varphi}^{M}(\Omega)$, we obtain the Burkholder-Davis-Gundy inequality on $L^{\varphi}(\Omega)$. Finally, using the previous martingale inequalities, some applications in Fourier analysis are presented in this article. We prove that the maximal Fejér operator is bounded from $H_{\varphi}[0,1)$ to $L^{\varphi}[0,1)$. As a consequence, we obtain several convergence results on both the partial sums and the Fejér means of the Walsh-Fourier series. In particular, both the boundedness of the maximal Fejér operator and the convergence results are new even for the weighted Hardy as well as for the (weighted) Orlicz Hardy spaces.

To be precise, this article is organized as follows.

In Section 2, we first recall some notation and notions on martingale theory, Musielak-Orlicz functions and weights. Then we give some properties and examples of Musielak-Orlicz functions.

In Section 3, with the help of [47, Theorem 2.7], we first prove that the Doob maximal operator $M$ is bounded on $L^{\varphi}(\Omega)$; see Theorem 3.2 below. Via this, we prove the weak type inequality and the dual version of the Doob maximal inequality; see Theorems 3.3 and 3.5 below. Then, using the extrapolation theorem (see Theorem 3.9 below, which is a variant of [16, Theorem 3.9] on probability spaces), we obtain the weighted Fefferman-Stein inequality for the Doob maximal operator; see Theorem 3.11 below. By establishing a vector-valued version (see Theorem 3.10 below) of the extrapolation theorem (see Theorem 3.9), we also obtain the Fefferman-Stein vectorvalued inequality on $L^{\varphi}(\Omega)$ (see Theorem 3.12 below). The extrapolation theorems in $L^{\varphi}\left(\mathbb{R}^{d}\right)$ were proved by Cruz-Uribe and Hästö [15]. However, the extrapolation theorems in [15] need to use the fact that the maximal operator is bounded on the dual spaces. It is hard to obtain an explicit expression of the dual space of $L^{\varphi}(\Omega)$ and hence the boundedness of the maximal operator on it is difficult to obtain. However, the dual space of the weighted Lebesgue space $L^{p}(\Omega, w d \mathbb{P})$ is already known (see, for example, [16, Theorem 3.9]), which enables us to obtain the extrapolation theorem for the weighted Lebesgue space $L^{p}(\Omega, w d \mathbb{P}$ ) (see Theorem 3.10). Using both this and an interpolation theorem of sublinear operator on $L^{\varphi}(\Omega)$ (see Theorem 3.1 below), we prove Theorem 3.12. Remarkably, our method skillfully avoids the requirement that the Doob maximal operator is bounded on the dual space of $L^{\varphi}(\Omega)$. It should be mentioned that Theorem 3.12 is a probabilistic 
version of [15, Corollary 6.1] and Theorem 3.12 covers the unknown weighted Orlicz case; see Remark 3.13. Via Theorem 3.12, we totally cover the Stein inequality [71, Theorem 3.8], which is further generalized to the Musielak-Orlicz case; see Theorem 3.14 below.

The target of Section 4 is to establish the atomic characterizations of five Musielak-Orlicz martingale Hardy spaces, $H_{\varphi}^{M}(\Omega), P_{\varphi}(\Omega), H_{\varphi}^{s}(\Omega), H_{\varphi}^{S}(\Omega)$ and $Q_{\varphi}(\Omega)$; see Theorems 4.1, 4.4 and 4.6 below. The above five martingale Hardy spaces include weighted martingale Hardy spaces, martingale Orlicz-Hardy spaces in [52], weighted martingale Orlicz-Hardy spaces, and variable martingale Hardy spaces in $[38,40]$ as special cases (see also Remark 2.2 below for more details). Unlike the classical case [80, Theorem 1.3.17], we introduce a new type of atoms which allow us to eliminate the inaccuracy of the growth properties of $\varphi$, which is totally different from the articles [76, 29, 36, 75, 52, 79]. This is a key idea to improve martingale inequalities [79, Theorem 1.9]. Moreover, our atomic characterizations of Musielak-Orlicz martingale Hardy spaces totally cover the variable martingale Hardy spaces, the weighted martingale Hardy spaces and the weighted martingale Orlicz-Hardy spaces; see Remark 4.8 below. In particular, the atomic characterizations of $H_{\varphi}^{M}(\Omega)$ and $H_{\varphi}^{S}(\Omega)$ are new even for martingale Hardy spaces. It should be point out that the classical argument used in the proof of [77, Theorem 2.2] does not work for $H_{\varphi}^{M}(\Omega)$ and $H_{\varphi}^{S}(\Omega)$. To overcome this difficulty, we construct appropriate stopping times under the regularity condition (see Lemma 4.7 below), which is another key idea of this article.

Section 5 is devoted to proving the Burkholder-Davis-Gundy inequality and to improving [79, Theorem 1.9]; see Theorems 5.7, 5.12 and 5.16 below. To be precise, via atomic characterizations, we first investigate some $\sigma$-sublinear operators defined on weighted martingale Hardy spaces; see Theorems 5.2 and 5.3 below. Three important examples of such operators are $S, s$ and $M$, respectively, as in (2.1), (2.2) and (1.1). Then we establish the relationships among five Musielak-Orlicz martingale Hardy spaces $H_{\varphi}^{M}(\Omega), P_{\varphi}(\Omega), H_{\varphi}^{s}(\Omega), H_{\varphi}^{S}(\Omega)$ and $Q_{\varphi}(\Omega)$; see Theorem 5.7 below. It is noteworthy that Theorem 5.7 totally improves Miyamoto et al. [52, Theorem 2.5 and Corollary 2.6] (see Remark 5.8 below). Then we prove the Burkholder-Davis-Gundy inequality in $L^{\varphi}(\Omega)$ (Theorems 5.12 and 5.16). Remarkably, an important tool in the proof of the BurkholderDavis-Gundy inequality (see [7, Theorem 1] and [41, Theorem 3]) is the extensions of the good- $\lambda$ inequalities, which invented by Burkholder and Gundy [11] (see also [9, Lemma 7.1]). However, good- $\lambda$ inequalities do not work anymore in the present setting. The reason behind this is that the space variant $x$ and the growth variant $t$ appeared in the considered Musielak-Orlicz function $\varphi(x, t)$ are inseparable. Using the dual version of the Doob maximal inequality and the Davis decomposition of martingale Musielak-Orlicz Hardy spaces, we give the proof of Theorems 5.12 and 5.16. Our method is different from the classical proofs of [9, Theorem 15.1] and [7, Theorem 1] (see also [50, Theorem 6.6.9]), because we did not use the good- $\lambda$ inequality. Despite the fact that our Burkholder-Davis-Gundy inequality covers several cases (see Example 2.16), the assumptions are not stronger than that of Bonami and Lépingle [7, Theorem 1]; see Remark 5.18 below. Using the Doob maximal inequality and the Burkholder-Gundy inequality, we then prove that the martingale transform operator is bounded on $L^{\varphi}(\Omega)$ (see Theorem 5.21 below).

We point out, under the condition that $\varphi$ is of uniformly lower type $p_{\varphi}^{-} \in(0,1)$ and upper type $p_{\varphi}^{+}=1$ (see Definition 2.9 below for their definitions), Xie et al. [79, Theorems 1.4, 1.5 and 1.9] established the atomic characterizations of martingale Musielak-Orlicz Hardy spaces $H_{\varphi}^{S}(\Omega)$, $P_{\varphi}(\Omega)$ and $Q_{\varphi}(\Omega)$, and further explored the relationships among five martingale Musielak-Orlicz Hardy spaces $H_{\varphi}^{M}(\Omega), P_{\varphi}(\Omega), H_{\varphi}^{s}(\Omega), H_{\varphi}^{S}(\Omega)$ and $Q_{\varphi}(\Omega)$. In this article, via introducing a new 
kind of atoms (see Definition 2.3 below), we then remove the above restriction in [79] that $\varphi$ is of uniformly lower type $p_{\varphi}^{-} \in(0,1)$ and upper type $p_{\varphi}^{+}=1$ (see Theorems 4.1 and 4.4 below). Moreover, differently from [79], we also establish the atomic characterizations of $H_{\varphi}^{S}(\Omega)$ and $H_{\varphi}^{M}(\Omega)$ (see Theorem 4.6 below). These improved (or new) atomic characterizations further induce the corresponding improvement on the relationships among these martingale Musielak-Orlicz Hardy spaces. Here, we also allow that the considered Musielak-Orlicz function is of the uniformly lower type index $p_{\varphi}^{-} \in(0, \infty)$ and the uniformly upper type index $p_{\varphi}^{+} \in(0, \infty)$, which cause some extra difficulties, because now these martingale Musielak-Orlicz Hardy spaces have wider generality than those in [79], which cover, for example, all weighted martingale Hardy spaces and weighted martingale Orlicz-Hardy spaces, while those martingale Musielak-Orlicz Hardy spaces in [79] cover only part of them. We point out that both the new atomic characterizations of these martingale Musielak-Orlicz Hardy spaces and the technical lemma on appropriate stopping times (Lemma 4.7) established in Section 4 play an essential role in overcoming these extra difficulties.

In Section 6, we introduce the Walsh system and the Fejér means. We then prove that the partial sum of the Walsh-Fourier series is uniformly bounded in $L^{\varphi}[0,1)$ (see Theorem 6.1 below). Moreover, we show that the Walsh-Fourier series is converges in the $L^{\varphi}[0,1)$-norm (see Corollary 6.2 below).

Finally, in Section 7, we prove that the maximal Fejér operator is bounded from the MusielakOrlicz Hardy space $H_{\varphi}[0,1)$ to $L^{\varphi}[0,1)$ (see Theorem 7.7 below). Theorem 7.7 is new even for the weighted Hardy spaces as well as for (weighted) Orlicz Hardy spaces (see Theorems 7.9 and 7.10). Moreover, we also obtain the consequences about the convergence of the Fejér means $\left(\sigma_{n} f\right)_{n \in \mathbb{N}}$ of a martingale $f$ (see Corollary 7.11 below).

Now, we fix some conventions on notation used in this article. Throughout the article, we always let $\mathbb{N}:=\{1,2, \ldots\}, \mathbb{Z}_{+}:=\mathbb{N} \cup\{0\}$ and $C$ denote a positive constant, which may vary from line to line. For any $p \in(0, \infty)$, we denote by $p^{\prime}$ the conjugate exponent to $p$, namely, $1 / p+1 / p^{\prime}=1$. We use the symbol $f \lesssim g$ to denote that there exists a positive constant $C$ such that $f \leq C g$. The symbol $f \sim g$ is used as an abbreviation of $f \lesssim g \lesssim f$. We also use the following convention: If $f \leq C g$ and $g=h$ or $g \leq h$, we then write $f \lesssim g \sim h$ or $f \lesssim g \lesssim h$, rather than $f \lesssim g=h$ or $f \lesssim g \leq h$. For any subset $E$ of $\Omega$, we use $\mathbf{1}_{E}$ to denote its characteristic function.

\section{Preliminaries}

This section includes some basic notions and lemmas used in later sections.

Denote by $\mathcal{M}$ the set of all martingales $f:=\left(f_{n}\right)_{n \in \mathbb{Z}_{+}}$related to $\left\{\mathcal{F}_{n}\right\}_{n \in \mathbb{Z}_{+}}$such that $f_{0}=0$. Let $\mathcal{T}$ be the set of all stopping times related to $\left\{\mathcal{F}_{n}\right\}_{n \in \mathbb{Z}_{+}}$. For any $f \in \mathcal{M}$ and $v \in \mathcal{T}$, we write $f^{v}:=\left\{f_{v \wedge n}\right\}_{n \in \mathbb{Z}_{+}}$to denote the stopped martingale and $B_{v}:=\{x \in \Omega: v(x)<\infty\}$.

Now we recall the definition of the martingale Musielak-Orlicz Hardy spaces. For any $f \in \mathcal{M}$, denote its martingale difference by

$$
d_{n} f:=f_{n}-f_{n-1}, \quad \forall n \in \mathbb{N} .
$$

Then the quadratic variations $S_{n}(f)$ and $S(f)$, and the conditional quadratic variations $s_{n}(f)$ and 
$s(f)$ of a martingale $f$ are defined, respectively, by setting, for any $n \in \mathbb{N}$,

$$
\begin{gathered}
S_{n}(f):=\left(\sum_{i=1}^{n}\left|d_{i} f\right|^{2}\right)^{\frac{1}{2}}, \quad S(f):=\left(\sum_{i=1}^{\infty}\left|d_{i} f\right|^{2}\right)^{\frac{1}{2}}, \\
s_{n}(f):=\left(\sum_{i=1}^{n} \mathbb{E}_{i-1}\left|d_{i} f\right|^{2}\right)^{\frac{1}{2}} \text { and } \quad s(f):=\left(\sum_{i=1}^{\infty} \mathbb{E}_{i-1}\left|d_{i} f\right|^{2}\right)^{\frac{1}{2}} .
\end{gathered}
$$

Let $\Lambda$ be the collection of all nondecreasing, nonnegative and adapted sequences $\left(\lambda_{n}\right)_{n \in \mathbb{Z}_{+}}$of functions. Recall that a sequence $\left(\lambda_{n}\right)_{n \in \mathbb{Z}_{+}}$of functions is said to be adapted if, for any $n \in \mathbb{Z}_{+}, \lambda_{n}$ is $\mathcal{F}_{n}$ measurable. Let $\lambda_{\infty}:=\lim _{n \rightarrow \infty} \lambda_{n}$. For any $f \in \mathcal{M}$, let

$$
\Lambda\left[P_{\varphi}\right](f):=\left\{\left(\lambda_{n}\right)_{n \in \mathbb{Z}_{+}} \in \Lambda:\left|f_{n}\right| \leq \lambda_{n-1} \text { for any } n \in \mathbb{N}, \quad \lambda_{\infty} \in L^{\varphi}(\Omega)\right\}
$$

and

$$
\Lambda\left[Q_{\varphi}\right](f):=\left\{\left(\lambda_{n}\right)_{n \in \mathbb{Z}_{+}} \in \Lambda: S_{n}(f) \leq \lambda_{n-1} \text { for any } n \in \mathbb{N}, \lambda_{\infty} \in L^{\varphi}(\Omega)\right\}
$$

Definition 2.1. Let $\varphi$ be a Musielak-Orlicz function. The martingale Musielak-Orlicz Hardy spaces $H_{\varphi}^{*}(\Omega), H_{\varphi}^{S}(\Omega), H_{\varphi}^{s}(\Omega), P_{\varphi}(\Omega)$ and $Q_{\varphi}(\Omega)$ are defined, respectively, as follows:

$$
\begin{gathered}
H_{\varphi}^{M}(\Omega):=\left\{f \in \mathcal{M}:\|f\|_{H_{\varphi}^{M}(\Omega)}:=\|M(f)\|_{L^{\varphi}(\Omega)}<\infty\right\}, \\
H_{\varphi}^{S}(\Omega):=\left\{f \in \mathcal{M}:\|f\|_{H_{\varphi}^{S}(\Omega)}:=\|S(f)\|_{L^{\varphi}(\Omega)}<\infty\right\}, \\
H_{\varphi}^{s}(\Omega):=\left\{f \in \mathcal{M}:\|f\|_{H_{\varphi}^{s}(\Omega)}:=\|s(f)\|_{L^{\varphi}(\Omega)}<\infty\right\}, \\
P_{\varphi}(\Omega):=\left\{f \in \mathcal{M}:\|f\|_{P_{\varphi}(\Omega)}:=\inf _{\left.\left(\lambda_{n}\right)_{n \in \mathbb{Z}_{+} \in \Lambda\left[P_{\varphi}(\Omega)\right]}\left\|\lambda_{\infty}\right\|_{L^{\varphi}(\Omega)}<\infty\right\}}\right.
\end{gathered}
$$

and

$$
Q_{\varphi}(\Omega):=\left\{f \in \mathcal{M}:\|f\|_{Q_{\varphi}(\Omega)}:=\inf _{\left(\lambda_{n}\right)_{n \in \mathbb{Z}_{+}} \in \Lambda\left[Q_{\varphi}(\Omega)\right]}\left\|\lambda_{\infty}\right\|_{L^{\varphi}(\Omega)}<\infty\right\} .
$$

Remark 2.2. The above five martingale Musielak-Orlicz Hardy spaces are the generalization of several known martingale Hardy spaces. For example, let $p \in(0, \infty), w$ be a weight, $\Phi$ an Orlicz function on $(0, \infty)$ and $p(\cdot): \Omega \rightarrow[1, \infty]$ a measurable function. If $\varphi(x, t):=w(x) t^{p}, \Phi(t), t^{p(x)}$ or $w(x) \Phi(t)$ for any $x \in \Omega$ and $t \in(0, \infty)$, then the corresponding martingale Musielak-Orlicz Hardy space becomes, respectively, the weighted martingale Hardy space, the martingale Orlicz-Hardy space (see [52, p. 671]), the variable martingale Hardy space (see [40, Chapter 2]) or the weighted martingale Orlicz-Hardy space. 
Definition 2.3. Let $\varphi$ be a Musielak-Orlicz function. A measurable function $a$ is called a $(\varphi, \infty)_{s^{-}}$ atom if there exists a stopping time $v$ related to $\left\{\mathcal{F}_{n}\right\}_{n \in \mathbb{Z}_{+}}(v$ is called the stopping time associated with $a$ ) such that

(i) $a_{n}:=\mathbb{E}_{n} a=0$ if $v \geq n$,

(ii) $\|s(a)\|_{L^{\infty}\left(B_{v}\right)} \leq\left\|\mathbf{1}_{B_{v}}\right\|_{L^{\varphi}(\Omega)}^{-1}$.

Similarly, $(\varphi, \infty)_{S}$-atoms and $(\varphi, \infty)_{M}$-atoms are defined, respectively, via replacing (ii) in the above definition by

$$
\|S(a)\|_{L^{\infty}\left(B_{v}\right)} \leq\left\|\mathbf{1}_{B_{v}}\right\|_{L^{\varphi}(\Omega)}^{-1}
$$

and

$$
\|M(a)\|_{L^{\infty}\left(B_{v}\right)} \leq\left\|\mathbf{1}_{B_{v}}\right\|_{L^{\varphi}(\Omega)}^{-1} .
$$

Let $r \in(0, \infty)$. Denote by $\mathcal{A}_{s}(\varphi, \infty)$ (resp., $\mathcal{A}_{S}(\varphi, \infty)$ or $\left.\mathcal{A}_{M}(\varphi, \infty)\right)$ the set of all sequences of triples $\left\{\mu^{k}, a^{k}, v^{k}\right\}_{k \in \mathbb{Z}}$, where $\left\{\mu^{k}\right\}_{k \in \mathbb{Z}}$ are non-negative real numbers, $\left\{a^{k}\right\}_{k \in \mathbb{Z}}$ are $(\varphi, \infty)_{s}$-atoms (resp., $(\varphi, \infty)_{S}$-atoms or $(\varphi, \infty)_{M}$-atoms), and $\left\{v^{k}\right\}_{k \in \mathbb{Z}} \subset \mathcal{T}$ satisfying (i) and (ii) of Definition 2.3, and also

$$
\left\|\left\{\sum_{k \in \mathbb{Z}}\left[\frac{\mu^{k} \mathbf{1}_{B_{v^{k}}}}{\left\|\mathbf{1}_{B_{v^{k}}}\right\|_{L^{\varphi}(\Omega)}}\right]^{r}\right\}^{\frac{1}{r}}\right\|_{L^{\varphi}(\Omega)}<\infty .
$$

Definition 2.4. Let $\varphi$ be a Musielak-Orlicz function and $r \in(0, \infty)$. The atomic martingale Musielak-Orlicz Hardy space $H_{\mathrm{at}, r}^{\varphi, \infty}(\Omega)\left(\operatorname{resp} ., H_{\mathrm{at}, r}^{\varphi, \infty, S}(\Omega), H_{\mathrm{at}, r}^{\varphi, \infty}(\Omega)\right)$ is defined to be the space of all $f \in \mathcal{M}$ satisfying that there exists a sequence of triples, $\left\{\mu^{k}, a^{k}, v^{k}\right\}_{k \in \mathbb{Z}} \in \mathcal{A}_{s}(\varphi, \infty)$ (resp., $\mathcal{A}_{S}(\varphi, \infty)$ or $\left.\mathcal{A}_{M}(\varphi, \infty)\right)$, such that, for any $n \in \mathbb{Z}_{+}$,

$$
\sum_{k \in \mathbb{Z}} \mu^{k} a_{n}^{k}=f_{n}
$$

Moreover, let

$$
\|f\|_{H_{\mathrm{at}, r}^{\varphi, \infty, s}(\Omega)}\left(\operatorname{resp} .,\|f\|_{H_{\mathrm{at}, r}^{\varphi, \infty, S}(\Omega)},\|f\|_{H_{\mathrm{at}, r}^{\varphi, \infty, M}(\Omega)}\right):=\inf \left\{\left\|\left[\sum_{k \in \mathbb{Z}}\left\{\frac{\mu^{k} \mathbf{1}_{B_{v^{k}}}}{\left\|\mathbf{1}_{B_{v^{k}}}\right\|_{L^{\varphi}(\Omega)}}\right\}^{r}\right]^{\frac{1}{r}}\right\|_{L^{\varphi}(\Omega)}\right\}<\infty
$$

where the infimum is taken over all decompositions of $f$ as above.

The stochastic basis $\left\{\mathcal{F}_{n}\right\}_{n \in \mathbb{Z}_{+}}$is said to be regular if there exists a positive constant $R$ such that, for any $n \in \mathbb{N}$,

$$
f_{n} \leq R f_{n-1}
$$

holds true for any nonnegative martingale $\left(f_{n}\right)_{n \in \mathbb{Z}_{+}}$.

The weights we consider in this article are special weights, that is, the martingales generated by a strictly positive $\varphi \in L^{1}(\Omega)$. To be precise, let $\varphi(\cdot, t):=\left\{\varphi_{n}(\cdot, t)\right\}_{n \in \mathbb{Z}_{+}}$be the martingale generated by $\varphi(\cdot, t)$ for any $t \in[0, \infty)$. For simplicity, we still use $\varphi(\cdot, t)$ to denote the martingale $\varphi(\cdot, t):=\left\{\varphi_{n}(\cdot, t)\right\}_{n \in \mathbb{Z}_{+}} \cdot$

The definition of $A_{p}$ weights for martingales was introduced by Izumisawa and Kazamaki in [33], which is now generalized to the Musielak-Orlicz case as follows. 
Definition 2.5. Let $q \in[1, \infty)$. A positive Musielak-Orlicz function $\varphi: \Omega \times[0, \infty) \rightarrow[0, \infty)$ is said to satisfy the uniformly $A_{q}(\Omega)$ condition if there exists a positive constant $K$ such that, when $q \in(1, \infty)$

$$
\sup _{t \in(0, \infty)} \mathbb{E}_{n}(\varphi)(\cdot, t)\left[\mathbb{E}_{n}\left(\varphi^{-\frac{1}{q-1}}\right)(\cdot, t)\right]^{q-1} \leq K \quad \mathbb{P} \text {-almost everywhere, } \quad \forall n \in \mathbb{Z}_{+}
$$

and, when $q=1$,

$$
\sup _{t \in(0, \infty)} \mathbb{E}_{n}(\varphi)(\cdot, t) \frac{1}{\varphi(\cdot, t)} \leq K \quad \mathbb{P} \text {-almost everywhere, } \quad \forall n \in \mathbb{Z}_{+}
$$

$\varphi$ is said to satisfy $A_{\infty}(\Omega)$ if $\varphi \in A_{q}(\Omega)$ for some $q \in[1, \infty)$.

It is easy to see that, for any $p, q \in(1, \infty)$ with $p \leq q, A_{1}(\Omega) \subset A_{p}(\Omega) \subset A_{q}(\Omega) \subset A_{\infty}(\Omega)$. Assuming that $\varphi$ is a Musielak-Orlicz function, let

$$
q(\varphi):=\inf \left\{q \in[1, \infty): \varphi \in A_{q}(\Omega)\right\} .
$$

The following $\mathbb{S}$ condition appears naturally in the weighted martingale inequalities. For more discussions, see Doléans-Dade and Meyer [17] and also Bonami and Lépingle [7].

Definition 2.6. Let $t \in[0, \infty)$. The martingale $\varphi(\cdot, t):=\left\{\varphi_{n}(\cdot, t)\right\}_{n \in \mathbb{Z}_{+}}$is said to satisfy the uniformly $\mathbb{S}$ condition, denoted by $\varphi \in \mathbb{S}$, if there exists a positive constant $K$ such that, for any $t \in[0, \infty)$, $n \in \mathbb{N}$ and almost every $\omega \in \Omega$,

$$
\frac{1}{K} \varphi_{n-1}(\omega, t) \leq \varphi_{n}(\omega, t) \leq K \varphi_{n-1}(\omega, t)
$$

The conditions $\mathbb{S}^{-}$and $\mathbb{S}^{+}$denote two parts of $\mathbb{S}$ satisfying only the left or the right hand side of the preceding inequalities, respectively.

The following lemma comes from Doléans-Dade and Meyer [17] (see also [50, Corollary $6.3 .3])$.

Lemma 2.7. Let $p \in(1, \infty)$ and $w$ be a weight. If $w \in A_{p}(\Omega) \cap \mathbb{S}^{-}$, then there exists a positive constant $\varepsilon$ such that $w \in A_{p-\varepsilon}(\Omega)$.

Bonami and Lépingle [7, Section 3] gave an example to illustrate that there exists a weight $w \in A_{p}(\Omega)$, while $w \notin \bigcup_{\varepsilon \in(0, \infty)} A_{p-\varepsilon}(\Omega)$.

Lemma 2.8. Let $\varphi$ be a Musielak-Orlicz function and $\varphi \in A_{\infty}(\Omega)$. If the stochastic basis $\left\{\mathcal{F}_{n}\right\}_{n \in \mathbb{Z}_{+}}$ is regular, then $\varphi \in \mathbb{S}$.

Proof. Since $\varphi \in A_{\infty}(\Omega)$, it follows that there exists an index $p \in(1, \infty)$ such that $\varphi \in A_{p}(\Omega)$. For any $x \in \Omega$ and $t \in(0, \infty)$, let

$$
\widehat{\varphi}(x, t):=[\varphi(x, t)]^{-\frac{1}{p-1}} \quad \text { and } \quad \widehat{\varphi}_{n}(x, t):=\mathbb{E}_{n}(\widehat{\varphi}(\cdot, t))(x), \quad n \in \mathbb{Z}_{+} .
$$


Then, by the Hölder inequality of the conditional expectation and $\varphi \in A_{p}(\Omega)$, we find that there exists a positive constant $K$ such that, for any $n \in \mathbb{Z}_{+}$,

$$
1=\left[\mathbb{E}_{n}\left(\varphi^{\frac{1}{p}} \varphi^{-\frac{1}{p}}\right)\right]^{p} \leq \varphi_{n}\left[\mathbb{E}_{n}\left(\varphi^{-\frac{1}{p-1}}\right)\right]^{p-1}=\varphi_{n} \widehat{\varphi}_{n}^{p-1} \leq K
$$

From this and the regularity, we deduce that, for any $n \in \mathbb{N}$,

$$
\varphi_{n-1} \leq K \widehat{\varphi}_{n-1}^{1-p} \leq K R^{p-1} \widehat{\varphi}_{n}^{1-p} \leq K R^{p-1} \varphi_{n}
$$

where $R$ is a positive constant as in (2.4). This implies that $\varphi \in \mathbb{S}^{-}$. Notice that the right hand side of (2.5) follows from the regularity condition (2.4). Thus, we have $\varphi \in \mathbb{S}$, which completes the proof of Lemma 2.8 .

Definition 2.9. Let $\varphi$ be a Musielak-Orlicz function. For any $p \in(0, \infty), \varphi$ is said to be of uniformly lower (resp., upper) type $p$ if there exists a positive constant $C_{(p)}$, depending on $p$, such that, for any $x \in \Omega$ and $t \in[0, \infty), s \in(0,1)$ (resp., $s \in[1, \infty)$ ),

$$
\varphi(x, s t) \leq C_{(p)} s^{p} \varphi(x, t) .
$$

Remark 2.10. Obviously, if $\varphi$ is both of uniformly lower type $p_{1}$ and of uniformly upper type $p_{2}$, then $p_{1} \leq p_{2}$. Moreover, if $\varphi$ is of uniformly lower (resp., upper) type $p$, then, it is also of uniformly lower (resp., upper) type $\widetilde{p}$ for any $\widetilde{p} \in(0, p)$ (resp., $\widetilde{p} \in(p, \infty))$.

Definition 2.11. Let $\varphi$ be a Musielak-Orlicz function. For any given $r \in(0, \infty)$, let

$$
\varphi_{r}(x, t):=\varphi\left(x, t^{r}\right), \quad \forall x \in \Omega, \quad \forall t \in[0, \infty) .
$$

The following lemma can be proved with standard arguments, the details being omitted.

Lemma 2.12. Let $\varphi$ be a Musielak-Orlicz function. Then, for any measurable function $f$ and $r \in(0, \infty)$, it holds true that

$$
\left\||f|^{r}\right\|_{L^{\varphi}[0,1)}=\|f\|_{L^{\varphi_{r}[0,1)}}^{r} .
$$

Lemma 2.13. Assumed that $r \in(0, \infty)$. If $\varphi$ is a Musielak-Orlicz function of uniformly lower (resp., upper) type $p \in(0, \infty)$, then $\varphi_{r}$ is of uniformly lower (resp., upper) type $p r$.

Proof. The desired result follows from the fact that, for any $x \in \Omega, s \in(0,1)$ and $t \in(0, \infty)$,

$$
\varphi_{r}(x, s t)=\varphi\left(x, s^{r} t^{r}\right) \leq C_{(p)} s^{p r} \varphi\left(x, t^{r}\right)=C_{(p)} s^{p r} \varphi_{r}(x, t),
$$

where $C_{(p)}$ is the positive constant as in (2.6). This finishes the proof of Lemma 2.13.

Definition 2.14. Let $\varphi$ be a Musielak-Orlicz function. Then the function

$$
\varphi^{*}(x, t)=\sup _{u \in(0, \infty)}[u t-\varphi(x, u)], \quad \forall x \in \Omega, \quad \forall t \in(0, \infty),
$$

is said to be complimentary to $\varphi$. 
Let us denote by $\varphi_{r}^{*}$ the complimentary function to $\varphi_{r}$.

The following lemma gives the relationship between the Musielak-Orlicz function and its complimentary function.

Lemma 2.15. Suppose that $\varphi$ is a Musielak-Orlicz function and $\varphi^{*}$ its complimentary function. If $\varphi$ is of uniformly lower (resp., upper) type $p$ with $p \in(1, \infty)$, then $\varphi^{*}$ is of uniformly upper (resp., lower) type $p^{\prime}$.

Proof. Suppose that $\varphi$ is of uniformly lower type $p$, namely, there exists a positive constant $C_{(p)}$ such that

$$
\varphi(x, s t) \leq C_{(p)} s^{p} \varphi(x, t), \quad \forall x \in \Omega, \forall s \in(0,1], \forall t \in(0, \infty)
$$

or, equivalently,

$$
\varphi(x, t) \leq C_{(p)} s^{p} \varphi(x, t / s), \quad \forall x \in \Omega, \forall s \in(0,1], \forall t \in(0, \infty) .
$$

We may suppose that $C_{(p)} \in[1, \infty)$. Let $\tilde{\varphi}(x, t):=C_{(p)} s^{p} \varphi(x, t / s)$ for any $x \in \Omega, t \in(0, \infty)$ and $s \in(0,1]$ and $(\tilde{\varphi})^{*}$ be its complementary function. By (2.7), we know that, for any $x \in \Omega$, $t \in(0, \infty)$ and $s \in(0,1]$,

$$
\begin{aligned}
(\tilde{\varphi})^{*}(x, t) & =\sup _{u \in(0, \infty)}[u t-\tilde{\varphi}(x, u)]=\sup _{u \in(0, \infty)}\left[u t-C_{(p)} s^{p} \varphi(x, u / s)\right] \\
& =\sup _{u \in(0, \infty)}\left[s t u-C_{(p)} s^{p} \varphi(x, u)\right]=C s^{p} \sup _{u \in(0, \infty)}\left[C_{(p)}^{-1} s^{1-p} t u-\varphi(x, u)\right] \\
& =C_{(p)} s^{p} \varphi^{*}\left(x, C^{-1} s^{1-p} t\right) .
\end{aligned}
$$

Since $\varphi(x) \leq \tilde{\varphi}(x)$, from (2.7), it follows that $(\tilde{\varphi})^{*}(x, t) \leq \varphi^{*}(x, t)$. Thus, we obtain, for any $x \in \Omega$, $t \in(0, \infty)$ and $s \in(0,1]$,

$$
C_{(p)} s^{p} \varphi^{*}\left(x, C_{(p)}^{-1} s^{1-p} t\right) \leq \varphi^{*}(x, t) .
$$

This further implies that, for any $x \in \Omega$ and $t \in(0, \infty)$,

$$
\varphi^{*}(x, v t) \leq C_{(p)}^{1 /(p-1)} v^{p /(p-1)} \varphi^{*}(x, t)=C_{(p)}^{1 /(p-1)} v^{p^{\prime}} \varphi^{*}(x, t), \quad \forall v \in\left[1 / C_{(p)}, \infty\right) .
$$

Since $C_{(p)} \in[1, \infty)$, this shows that $\varphi^{*}$ is of uniformly upper type $p^{\prime}$.

The desired result can be proved similarly when $\varphi$ is of uniformly upper type $p$. Indeed, similarly to (2.8), we conclude that, for any $x \in \Omega$ and $t \in(0, \infty)$,

$$
\varphi^{*}(x, v t) \leq C_{(p)}^{1 /(p-1)} v^{p^{\prime}} \varphi^{*}(x, t), \quad \forall v \in\left(0,1 / C_{(p)}\right] .
$$

Since $\varphi^{*}$ is increasing, we have, for any $v \in\left(\frac{1}{C_{(p)}}, 1\right]$ and $t \in(0, \infty)$,

$$
\varphi^{*}(x, v t) \leq \varphi^{*}(x, t) \leq C_{(p)}^{p^{\prime}} v^{p^{\prime}} \varphi^{*}(x, t)
$$

and hence $\varphi^{*}$ is of uniformly lower type $p^{\prime}$. This finishes the proof of Lemma 2.15.

Now we give some examples of Musielak-Orlicz functions with various properties. 
Example 2.16. All the following functions $\varphi$ are Musielak-Orlicz functions, where $w$ is a special weight on $\Omega$.

(i) For any given $p \in(1, \infty)$ and any $x \in \Omega$ and $t \in(0, \infty)$, let $\varphi(x, t):=w(x) t^{p} / p$ [or $\varphi(x, t):=$ $\left.w(x) t^{p}\right]$. Then $\varphi^{*}(x, t)=[w(x)]^{-\frac{1}{p-1}} t^{p^{\prime}} / p^{\prime}\left[\right.$ or $\left.\varphi^{*}(x, t)=[w(x)]^{-\frac{1}{p-1}} t^{p^{\prime}} / p^{\prime} p^{-\frac{1}{p-1}}\right]$ for any $x \in \Omega$ and $t \in(0, \infty)$, where $p^{\prime}$ is the conjugate index of $p$ and hence $\varphi^{*}$ is of uniformly lower type $p^{-}$for any $p^{-} \in\left(0, p^{\prime}\right]$ and of uniformly upper type $p^{+}$for any $p^{+} \in\left[p^{\prime}, \infty\right)$.

(ii) Let $\alpha \in(2, \infty)$ and $\varphi(x, t):=w(x) t^{\alpha}(1+|\log t|)$ for any $x \in \Omega$ and $t \in(0, \infty)$. It is not difficult to show that $\varphi$ is of uniformly lower type $\alpha-\varepsilon$ and of uniformly upper type $\alpha+\varepsilon$, where $\varepsilon$ is an arbitrary positive constant. By Remark 2.10 and Lemma 2.15, we know that $\varphi^{*}$ is of uniformly lower type $p^{-}$for any $p^{-} \in\left(0, \alpha^{\prime}\right)$ and of uniformly upper type $p^{+}$for any $p^{+} \in\left(\alpha^{\prime}, \infty\right)$.

(iii) Let $\varphi(x, t):=w(x)\left(e^{t}-t-1\right)$ for any $x \in \Omega$ and $t \in(0, \infty)$. Then we know that $\varphi$ is of uniformly lower type $p^{-}$for any $p^{-} \in(0,2]$. However, $\varphi$ does not have any uniformly upper type property.

(iv) Let $\alpha \in(1, \infty)$ and $\varphi(x, t):=w(x) t^{\alpha}(1+\log (1+t))$ for any $x \in \Omega$ and $t \in(0, \infty)$. Then $\varphi$ is of uniformly lower type $\alpha$ and of uniformly upper type $\alpha+\varepsilon$ for any $\varepsilon \in(0, \infty)$. Thus, $\varphi^{*}$ is of uniformly lower type $p^{-}$for any $p^{-} \in\left(0, \alpha^{\prime}\right)$ and of uniformly upper type $p^{+}$for any $p^{+} \in\left[\alpha^{\prime}, \infty\right)$.

(v) Let $\alpha \in[2, \infty)$ and $\varphi(x, t):=w(x) t^{\alpha} / \log (e+t)$ for any $x \in \Omega$ and $t \in(0, \infty)$. Then $\varphi$ is of uniformly lower type $\alpha-\varepsilon$ for any $\varepsilon \in(0, \infty)$ and of uniformly upper type $\alpha$. Thus, $\varphi^{*}$ is of uniformly lower type $p^{-}$for any $p^{-} \in\left(0, \alpha^{\prime}\right]$ and of uniformly upper type $p^{+}$for any $p^{+} \in\left(\alpha^{\prime}, \infty\right)$.

(vi) Let $\alpha \in(1, \infty), \beta \in(0, \infty), \gamma \in[0,2 \alpha(1+\log 2)]$ and

$$
\varphi(x, t):=\frac{t^{\alpha}}{(\log (e+x))^{\beta}+(\log (e+t))^{\gamma}}
$$

for any $x \in \Omega=[0,1)$ and $t \in(0, \infty)$. Then $\varphi \in A_{1}(\Omega)$, which is of uniformly lower type $\alpha-\varepsilon$ for any $\varepsilon \in(0, \infty)$ and of uniformly upper type $\alpha$. Thus, $\varphi^{*}$ is of uniformly lower type $p^{-}$for any $p^{-} \in\left(0, \alpha^{\prime}\right]$ and of uniformly upper type $p^{+}$for any $p^{+} \in\left(\alpha^{\prime}, \infty\right)$ (see Yang et al. $[80$, p. 11]).

(vii) Let $1<p \leq q<\infty$ and $\varphi(x, t):=t^{p}+w(x) t^{q}$ for any $x \in \Omega$ and $t \in(0, \infty)$. Then $\varphi$ is of uniformly lower type $p^{-}$for any $p^{-} \in(0, p]$ and of uniformly upper type $p^{+}$for any $p^{+} \in[q, \infty)$. Thus, $\varphi^{*}$ is of uniformly lower type $p^{-}$for any $p^{-} \in\left(0, q^{\prime}\right]$ and of uniformly upper type $p^{+}$for any $p^{+} \in\left[p^{\prime}, \infty\right)$. If $w \in A_{\infty}(\Omega)$, we claim that $\varphi \in A_{\infty}(\Omega)$. Indeed, since $w \in A_{\infty}(\Omega)$, it follows that there exists $q_{0} \in[1, \infty)$ such that $w \in A_{q_{0}}(\Omega)$. If $q_{0}=1$, it is clear that $\varphi \in A_{1}(\Omega)$. Now we assume that $q_{0} \in(1, \infty)$. Notice that, for any $n \in \mathbb{N}$,

$$
\frac{t^{p}+w_{n} t^{q}}{t^{p}+w t^{q}} \leq \mathbf{1}_{\left\{x \in \Omega: w_{n}(x)<w(x)\right\}}+\frac{1+w_{n} t^{q-p}}{1+w t^{q-p}} \mathbf{1}_{\left\{x \in \Omega: w_{n}(x) \geq w(x)\right\}} \leq 1+\frac{w_{n}}{w} .
$$


From this and $w \in A_{q_{0}}(\Omega)$, we deduce that

$$
\begin{aligned}
\sup _{t \in(0, \infty)} \mathbb{E}_{n}(\varphi)(\cdot, t)\left[\mathbb{E}_{n}\left(\varphi^{-\frac{1}{q_{0}-1}}\right)(\cdot, t)\right]^{q_{0}-1} & =\sup _{t \in(0, \infty)}\left[\mathbb{E}_{n}\left(\left(\frac{t^{p}+w_{n} t^{q}}{t^{p}+w t^{q}}\right)^{\frac{1}{q_{0}-1}}\right)\right]^{q_{0}-1} \\
& \lesssim 1+\left[\mathbb{E}_{n}\left(\left(\frac{w_{n}}{w}\right)^{\frac{1}{q_{0}-1}}\right)\right]^{q_{0}-1} \\
& \sim 1+\mathbb{E}_{n} w\left[\mathbb{E}_{n}\left(w^{-\frac{1}{q_{0}-1}}\right)\right]^{q_{0}-1} \lesssim 1,
\end{aligned}
$$

namely, $\varphi \in A_{q_{0}}(\Omega)$, which completes the proof of the above claim.

Example 2.17. Let $\varphi(x, t):=t^{p(x)}$ for any $x \in \Omega$ and $t \in(0, \infty)$,

$$
1<p_{1}:=\underset{x \in \Omega}{\operatorname{essinf}} p(x) \leq p_{2}:=\underset{x \in \Omega}{\operatorname{ess} \sup } p(x)<\infty .
$$

Then $\varphi^{*}(x, t)=t^{q(x)}$ for any $x \in \Omega$ and $t \in(0, \infty)$, where $\frac{1}{p(x)}+\frac{1}{q(x)}=1$. Thus, $\varphi$ is of uniformly lower type $p^{-}$for any $p^{-} \in\left(0, p_{1}\right]$ and of uniformly upper type $p^{+}$for any $p^{+} \in\left[p_{2}, \infty\right)$. However, $\varphi \notin A_{\infty}(\Omega)$ (see, for example, [81, Remark 2.23]).

The following key lemma was originated from [53, Theorem 13.18] and improved by [15, Lemma 2.7 and Remark 2.9].

Lemma 2.18. Let $\varphi$ be Musielak-Orlicz function and $\varphi^{*}$ its complimentary function. If $\varphi$ is of uniformly lower type 1, then there exists a positive constant $C$ such that, for any $f \in L^{\varphi}(\Omega)$,

$$
\frac{1}{C}\|f\|_{L^{\varphi}(\Omega)} \leq \sup _{g \in L^{\varphi^{*}}(\Omega),\|g\|_{L^{\varphi^{*}}(\Omega)} \leq 1} \int_{\Omega} f g d \mathbb{P} \leq 2\|f\|_{L^{\varphi}(\Omega)} .
$$

Notice that, if the Musielak-Orlicz function $\varphi$ is of uniformly lower type $p_{\varphi}^{-}$with $p_{\varphi}^{-} \in(1, \infty)$, then (2.9) holds true.

Actually, Lemma 2.18 was proved in [15] for Musielak-Orlicz functions $\varphi$ satisfying that there exists a positive constant $C$ such that, for any $0<s<t$ and $x \in \Omega$,

$$
\frac{\varphi(x, s)}{s} \leq C \frac{\varphi(x, t)}{t}
$$

We should point out that (2.10) holds true if and only if $\varphi$ is of uniformly lower type 1. Indeed, if $\varphi$ is of uniformly lower type 1 , then, for any $0<s<t$ and $x \in \Omega$,

$$
\varphi(x, s)=\varphi\left(x, \frac{s}{t} t\right) \leq C \frac{s}{t} \varphi(x, t) .
$$

Conversely, if (2.10) holds true with some positive constant $C$, then, for any $s \in(0,1), t \in(0, \infty)$ and $x \in \Omega$,

$$
\frac{\varphi(x, s t)}{s t} \leq C \frac{\varphi(x, t)}{t} \Longleftrightarrow \varphi(x, s t) \leq C s \varphi(x, t) .
$$




\section{The Doob maximal operator}

In this section, we explore the boundedness of the Doob maximal operator.

To prove the Doob maximal inequality for Musielak-Orlicz spaces, we need the following interpolation theorem about the sublinear operator on $L^{\varphi}(\Omega)$, which was proved in [47, Theorem 2.7] (see also [80, Theorem 2.1.1]).

Theorem 3.1. Let $p_{1}, p_{2} \in(0, \infty), p_{1}<p_{2}$ and $\varphi$ be a Musielak-Orlicz function with uniformly lower type $p_{\varphi}^{-}$and upper type $p_{\varphi}^{+}$. If $0<p_{1}<p_{\varphi}^{-} \leq p_{\varphi}^{+}<p_{2}<\infty$ and $T$ is a sublinear operator defined on $L^{p_{1}}(\Omega, \varphi(\cdot, 1) d \mathbb{P})+L^{p_{2}}(\Omega, \varphi(\cdot, 1) d \mathbb{P})$ satisfying that, for any $i \in\{1,2\}$ and any $\alpha \in(0, \infty), t \in(0, \infty)$,

$$
\varphi(\{x \in \Omega:|T(f)(x)|>\alpha\}, t) \leq C_{i} \alpha^{-p_{i}} \int_{\Omega}|f(x)|^{p_{i}} \varphi(x, t) d \mathbb{P},
$$

where $C_{i}$ is a positive constant independent of $f, t$ and $\alpha$. Then $T$ is bounded on $L^{\varphi}(\Omega)$ and, moreover, there exists a positive constant $C$ such that, for any $f \in L^{\varphi}(\Omega)$,

$$
\int_{\Omega} \varphi(x,|T(f)(x)|) d \mathbb{P} \leq C \int_{\Omega} \varphi(x,|f(x)|) d \mathbb{P} .
$$

Theorem 3.2. Let $\varphi \in A_{\infty}(\Omega)$ be a Musielak-Orlicz function with uniformly lower type $p_{\varphi}^{-}$and upper type $p_{\varphi}^{+}$. If

$$
q(\varphi)<p_{\varphi}^{-} \leq p_{\varphi}^{+}<\infty
$$

then the Doob maximal operator $M$ is bounded on $L^{\varphi}(\Omega)$ and, moreover, there exists a positive constant $C$ such that, for any $f \in L^{\varphi}(\Omega)$,

$$
\int_{\Omega} \varphi(x, M(f)(x)) d \mathbb{P}(x) \leq C \int_{\Omega} \varphi(x,|f(x)|) d \mathbb{P}(x) .
$$

Proof. From the definition of $q(\varphi)$, it follows that, for any $p_{1}, p_{2} \in(q(\varphi), \infty), \varphi \in A_{p_{1}}(\Omega)$ and $\varphi \in A_{p_{2}}(\Omega)$. Combining this and the weighted Doob maximal inequality (see [50, Theorem 6.6.3]), we find that, for any $f \in L^{p_{1}}(\Omega, \varphi(\cdot, 1) d \mathbb{P})+L^{p_{2}}(\Omega, \varphi(\cdot, 1) d \mathbb{P}), \alpha \in(0, \infty)$ and $t \in(0, \infty)$,

$$
\varphi(\{x \in \Omega: M(f)(x)>\alpha\}, t) \leq \alpha^{-p_{1}} \int_{\Omega}[M(f)(x)]^{p_{1}} \varphi(x, t) d \mathbb{P} \lesssim \alpha^{-p_{1}} \int_{\Omega}|f(x)|^{p_{1}} \varphi(x, t) d \mathbb{P}
$$

and

$$
\varphi(\{x \in \Omega: M(f)(x)>\alpha\}, t) \leq \alpha^{-p_{2}} \int_{\Omega}[M(f)(x)]^{p_{2}} \varphi(x, t) d \mathbb{P} \lesssim \alpha^{-p_{2}} \int_{\Omega}|f(x)|^{p_{2}} \varphi(x, t) d \mathbb{P} .
$$

From this, Theorem 3.1 and the fact that $M$ is a sublinear operator, we deduce that $M$ is bounded on $L^{\varphi}(\Omega)$, which completes the proof of Theorem 3.2.

As a consequence, we apply Theorem 3.2 to obtain the following weak type inequality of the Doob maximal operator on $L^{\varphi}(\Omega)$. 
Theorem 3.3. Let $\varphi \in A_{\infty}(\Omega)$ be a Musielak-Orlicz function with uniformly lower type $p_{\varphi}^{-}$and upper type $p_{\varphi}^{+}$satisfying (3.2). Then there exists a positive constant $C$ such that, for any $f \in L^{\varphi}(\Omega)$,

$$
\sup _{\rho \in(0, \infty)}\left[\rho\left\|\mathbf{1}_{\{x \in \Omega: M(f)(x)>\rho\}}\right\|_{L^{\varphi}(\Omega)}\right] \leq C\|f\|_{L^{\varphi}(\Omega)} .
$$

Proof. For any $f \in L^{\varphi}(\Omega)$ and $\rho \in(0, \infty)$, by Theorem 3.2, we know that there exists a constant $C \in(1, \infty)$ such that

$$
\begin{aligned}
\int_{\Omega} \varphi\left(x, \frac{\mathbf{1}_{\{x \in \Omega: M(f)(x)>\rho\}}(x)}{\|f\|_{L^{\varphi}(\Omega)} / \rho}\right) d \mathbb{P}(x) & =\int_{\{x \in \Omega: M(f)(x)>\rho\}} \varphi\left(x, \frac{\rho}{\|f\|_{L^{\varphi}(\Omega)}}\right) d \mathbb{P}(x) \\
& \leq \int_{\Omega} \varphi\left(x, \frac{M(f)(x)}{\|f\|_{L^{\varphi}(\Omega)}}\right) d \mathbb{P}(x) \leq C \int_{\Omega} \varphi\left(x, \frac{f(x)}{\|f\|_{L^{\varphi}(\Omega)}}\right) d \mathbb{P}(x)=C .
\end{aligned}
$$

Combining this and the uniformly lower type $p_{\varphi}^{-}$property of $\varphi$, we find that, for any $\rho \in(0, \infty)$,

$$
\int_{\Omega} \varphi\left(x,\left[C C_{\left(p_{\bar{\varphi}}^{-}\right)}\right]^{\frac{-1}{p_{\varphi}}} \frac{\mathbf{1}_{\{x \in \Omega: M(f)(x)>\rho\}}(x)}{\|f\|_{L^{\varphi}(\Omega)} / \rho}\right) d \mathbb{P}(x) \leq \frac{1}{C} \int_{\Omega} \varphi\left(x, \frac{\mathbf{1}_{\{x \in \Omega: M(f)(x)>\rho\}}(x)}{\|f\|_{L^{\varphi}(\Omega)} / \rho}\right) d \mathbb{P}(x) \leq 1 .
$$

Therefore, for any $\rho \in(0, \infty)$, we have

$$
\left\|\mathbf{1}_{\{x \in \Omega: M(f)(x)>\rho\}}\right\|_{L^{\varphi}(\Omega)} \leq \frac{1}{\rho}\left[C C_{\left(p_{\varphi}^{-}\right)}\right]^{\frac{1}{p_{\bar{\varphi}}^{-}}}\|f\|_{L^{\varphi}(\Omega)} .
$$

Thus, we obtain

$$
\sup _{\rho \in(0, \infty)}\left[\rho\left\|\mathbf{1}_{\{x \in \Omega: M(f)(x)>\rho\}}\right\|_{L^{\varphi}(\Omega)}\right] \leq\left[C C_{\left(p_{\varphi}^{-}\right)}\right]^{\frac{1}{p_{\varphi}^{-}}}\|f\|_{L^{\varphi}(\Omega)}
$$

This finishes the proof of Theorem 3.3.

Using Theorem 3.2, we also obtain the following corollary.

Corollary 3.4. Let $\varphi$ be a Musielak-Orlicz function with uniformly lower type $p_{\varphi}^{-}$and uniformly upper type $p_{\varphi}^{+}$. If $\varphi^{*} \in A_{\infty}(\Omega)$ satisfies

$$
q\left(\varphi^{*}\right)<\left(p_{\varphi}^{+}\right)^{\prime} \leq\left(p_{\varphi}^{-}\right)^{\prime}<\infty
$$

then the Doob maximal operator $M$ is bounded on $L^{\varphi^{*}}(\Omega)$.

Proof. Lemma 2.15 and (3.3) imply that (3.2) holds true for the function $\varphi^{*}$. Combining this and Theorem 3.2, we know that $M$ is bounded on $L^{\varphi^{*}}(\Omega)$. This finishes the proof of Corollary 3.4.

Now we turn to the dual version of Theorem 3.2. A detailed treatment of the following inequality for $L^{p}(\Omega)$ was given by Dilworth in [18]. Moreover, Burkholder et al. [10, Theorem 3.2] proved it in Orlicz spaces. Our result provides a general case of the dual version of the Doob maximal inequality. 
Theorem 3.5. Let $\varphi$ be a Musielak-Orlicz function with uniformly lower type $p_{\varphi}^{-}$for some $p_{\varphi}^{-} \in$ $[1, \infty)$. If the Doob maximal operator $M$ is bounded on $L^{\varphi^{*}}(\Omega)$, then there exists a positive constant $C$ such that, for any sequence $\left(g_{k}\right)_{k \in \mathbb{N}}$ of non-negative $\mathcal{F}$ measurable functions,

$$
\left\|\sum_{k \in \mathbb{N}} \mathbb{E}_{k}\left(g_{k}\right)\right\|_{L^{\varphi}(\Omega)} \leq C\left\|\sum_{k \in \mathbb{N}} g_{k}\right\|_{L^{\varphi}(\Omega)} .
$$

Proof. For any non-negative measurable function $f \in L^{\varphi^{*}}(\Omega)$ with $\|f\|_{L^{\varphi^{*}}(\Omega)} \leq 1$, by Remark 2.10, Lemma 2.18, the monotone convergence theorem and the assumed boundedness of $M$ on $L^{\varphi^{*}}(\Omega)$, we obtain

$$
\begin{aligned}
\int_{\Omega} \sum_{k \in \mathbb{N}} \mathbb{E}_{k}\left(g_{k}\right) f d \mathbb{P} & =\sum_{k \in \mathbb{N}} \int_{\Omega} \mathbb{E}_{k}\left(g_{k}\right) f d \mathbb{P}=\sum_{k \in \mathbb{N}} \int_{\Omega} g_{k} \mathbb{E}_{k}(f) d \mathbb{P} \\
& \leq \sum_{k \in \mathbb{N}} \int_{\Omega} g_{k} \sup _{k \in \mathbb{N}} \mathbb{E}_{k}(f) d \mathbb{P} \leq 2\left\|\sum_{k \in \mathbb{N}} g_{k}\right\|_{L^{\varphi}(\Omega)}\|M(f)\|_{L^{\varphi^{*}}(\Omega)} \\
& \lesssim\left\|\sum_{k \in \mathbb{N}} g_{k}\right\|_{L^{\varphi}(\Omega)}\|f\|_{L^{\varphi^{*}}(\Omega)} \lesssim\left\|\sum_{k \in \mathbb{N}} g_{k}\right\|_{L^{\varphi}(\Omega)} .
\end{aligned}
$$

From this, Lemma 2.18 and the fact that $\sum_{k \in \mathbb{N}} \mathbb{E}_{k}\left(g_{k}\right)$ is non-negative, the conclusion follows immediately. This finishes the proof of Theorem 3.5.

As an immediate consequence of Corollary 3.4 and Theorem 3.5, we have the following corollary.

Corollary 3.6. Let $\varphi$ be a Musielak-Orlicz function with uniformly lower type $p_{\varphi}^{-}$and uniformly upper type $p_{\varphi}^{+}$. If $\varphi^{*} \in A_{\infty}(\Omega)$ satisfies (3.3), then there exists a positive constant $C$ such that, for any sequence $\left(g_{k}\right)_{k \in \mathbb{N}}$ of non-negative $\mathcal{F}$ measurable functions,

$$
\left\|\sum_{k \in \mathbb{N}} \mathbb{E}_{k}\left(g_{k}\right)\right\|_{L^{\varphi}(\Omega)} \leq C\left\|\sum_{k \in \mathbb{N}} g_{k}\right\|_{L^{\varphi}(\Omega)} .
$$

Remark 3.7. In Theorem 3.5 and Corollary 3.6, the sequence $\left(g_{k}\right)_{k \in \mathbb{N}}$ is not assumed to be adapted.

Corollary 3.8. Let $\varphi$ be a Musielak-Orlicz function with uniformly lower type $p_{\varphi}^{-}$for some $p_{\varphi}^{-} \in$ $[2, \infty)$. If the Doob maximal operator $M$ is bounded on $L^{\varphi_{1 / 2}^{*}}(\Omega)$, then there exists a positive constant $C$ such that, for any $f \in \mathcal{M}$,

$$
\|f\|_{H_{\varphi}^{s}(\Omega)} \leq C\|f\|_{H_{\varphi}^{S}(\Omega)} .
$$

Proof. For any $f \in \mathcal{M}$ and $k \in \mathbb{Z}_{+}$, let $g_{k}:=\left|d_{k+1} f\right|^{2}$. Combining this, Lemmas 2.12 and 2.13, and Theorem 3.5, we find that

$$
\|f\|_{H_{\varphi}^{s}(\Omega)}=\|s(f)\|_{L^{\varphi}(\Omega)}=\left\|\left[\sum_{k \in \mathbb{Z}_{+}} \mathbb{E}_{k}\left(\left|d_{k+1} f\right|^{2}\right)\right]^{1 / 2}\right\|\left\|_{L^{\varphi}(\Omega)}=\right\| \sum_{k \in \mathbb{Z}_{+}} \mathbb{E}_{k}\left(\left|d_{k+1} f\right|^{2}\right) \|_{L^{\varphi_{1 / 2}(\Omega)}}^{1 / 2}
$$




$$
\lesssim\left\|\sum_{k \in \mathbb{Z}_{+}}\left|d_{k+1} f\right|^{2}\right\|_{L^{\varphi_{1 / 2}(\Omega)}}^{1 / 2} \sim\|S(f)\|_{L^{\varphi}(\Omega)} \sim\|f\|_{H_{\varphi}^{S}(\Omega)},
$$

which completes the proof of Corollary 3.8.

Let $p \in[1, \infty)$ and $w \in A_{p}(\Omega)$. Denote by $\mathcal{S}$ the set of pairs $(f, g)$ of nonnegative and measurable functions. If we write

$$
\int_{\Omega}[f(x)]^{p} w(x) d \mathbb{P}(x) \leq C \int_{\Omega}[g(x)]^{p} w(x) d \mathbb{P}(x), \quad \forall(f, g) \in \mathcal{S},
$$

we mean that the above inequality holds true for any pair $(f, g) \in \mathcal{S}$ and the positive constant $C$ depends only on $p$ and the $A_{p}(\Omega)$ constant of $w$ as in Definition 2.5.

The following extrapolation theorem, Theorem 3.9, plays a crucial role in the proof of the vector-valued extrapolation theorem, Theorem 3.10 below. The proof of Theorem 3.9 is similar to that of [16, Theorem 3.9], the details being omitted.

Theorem 3.9. Suppose that, for some $p_{0} \in[1, \infty)$ and $w_{0} \in A_{p_{0}}(\Omega)$, there exists a positive constant C such that

$$
\int_{\Omega}[f(x)]^{p_{0}} w_{0}(x) d \mathbb{P}(x) \leq C \int_{\Omega}[g(x)]^{p_{0}} w_{0}(x) d \mathbb{P}(x), \quad \forall(f, g) \in \mathcal{S} .
$$

Then, for any given $p \in(1, \infty)$ and $w \in A_{p}(\Omega)$, there exists a positive constant $C$ such that

$$
\int_{\Omega}[f(x)]^{p} w(x) d \mathbb{P}(x) \leq C \int_{\Omega}[g(x)]^{p} w(x) d \mathbb{P}(x), \quad \forall(f, g) \in \mathcal{S} .
$$

Theorem 3.10. Assume that $w_{0} \in A_{p_{0}}(\Omega)$ for some $p_{0} \in[1, \infty)$, and the set $\mathcal{S}$ satisfies (3.4) with some positive constant $C$. Then, for any given $p, r \in(1, \infty)$ and $w \in A_{p}(\Omega)$, there exists a positive constant $C$ such that, for any pair sequence $\left\{\left(f_{j}, g_{j}\right)\right\}_{j \in \mathbb{N}} \subset \mathcal{S}$,

$$
\left\|\left(\sum_{j \in \mathbb{N}} f_{j}^{r}\right)^{\frac{1}{r}}\right\|_{L^{p}(\Omega, w d \mathbb{P})} \leq C\left\|\left(\sum_{j \in \mathbb{N}} g_{j}^{r}\right)^{\frac{1}{r}}\right\|_{L^{p}(\Omega, w d \mathbb{P})} .
$$

Proof. For any given $r \in(1, \infty)$, let

$$
\mathcal{S}_{r}:=\left\{(F, G): F:=\left(\sum_{j \in \mathbb{N}} f_{j}^{r}\right)^{\frac{1}{r}}, G:=\left(\sum_{j \in \mathbb{N}} g_{j}^{r}\right)^{\frac{1}{r}},\left\{\left(f_{j}, g_{j}\right)\right\}_{j \in \mathbb{N}} \subset \mathcal{S}\right\} .
$$

Now, we claim that (3.4) holds true with $p_{0}=r$ for the set $\mathcal{S}_{r}$. Indeed, for any $(F, G) \in \mathcal{S}_{r}$, there exists a sequence $\left\{\left(f_{j}, g_{j}\right)\right\}_{j \in \mathbb{N}} \subset \mathcal{S}$ such that

$$
F=\left(\sum_{j \in \mathbb{N}} f_{j}^{r}\right)^{\frac{1}{r}} \text { and } \quad G=\left(\sum_{j \in \mathbb{N}} g_{j}^{r}\right)^{\frac{1}{r}}
$$


For any given $w \in A_{r}(\Omega)$, from Theorem 3.9 with $p=r$, it follows that

$$
\begin{aligned}
\int_{\Omega}[F(x)]^{r} w(x) d \mathbb{P}(x) & =\sum_{j \in \mathbb{N}} \int_{\Omega}\left[f_{j}(x)\right]^{r} w(x) d \mathbb{P}(x) \\
& \lesssim \sum_{j \in \mathbb{N}} \int_{\Omega}\left[g_{j}(x)\right]^{r} w(x) d \mathbb{P}(x) \sim \int_{\Omega}[G(x)]^{r} w(x) d \mathbb{P}(x),
\end{aligned}
$$

which proves the above claim. Using this claim and Theorem 3.9 for the set $\mathcal{S}_{r}$, we know that, for any given $p \in(1, \infty)$ and $w \in A_{p}(\Omega)$,

$$
\int_{\Omega}[F(x)]^{p} w(x) d \mathbb{P}(x) \lesssim \int_{\Omega}[G(x)]^{p} w(x) d \mathbb{P}(x), \quad \forall(F, G) \in \mathcal{S}_{r},
$$

which implies that (3.5) holds true with some positive constant $C$. This finishes the proof of Theorem 3.10.

Using Theorem 3.10, we obtain the following weighted Fefferman-Stein vector-valued inequality for the Doob maximal operator.

Theorem 3.11. Let $p \in(1, \infty)$ and $w \in A_{p}(\Omega)$. Then, for any given $r \in(1, \infty)$, there exists $a$ positive constant $C$ such that, for any sequence $\left\{f_{j}\right\}_{j \in \mathbb{N}}$ of measurable functions,

$$
\left\|\left\{\sum_{j \in \mathbb{N}}\left[M\left(f_{j}\right)\right]^{r}\right\}\right\|_{L^{p}(\Omega, w d \mathbb{P})}^{\frac{1}{r}} \leq C\left\|\left(\sum_{j \in \mathbb{N}}\left|f_{j}\right|^{r}\right)^{\frac{1}{r}}\right\|_{L^{p}(\Omega, w d \mathbb{P})} .
$$

Proof. Let $p \in(1, \infty)$. Denote the family of extrapolation pairs by

$$
\mathcal{S}:=\left\{(M(f),|f|): f \in L^{p}(\Omega, w d \mathbb{P})\right\} .
$$

Then, by the weighted Doob maximal inequality (see, for example, [50, Theorem 6.6.3]), we find that, for any given $p \in(1, \infty), w \in A_{p}(\Omega)$ and for the set $\mathcal{S}$, (3.4) holds true with some positive constant $C$. Applying Theorem 3.10, we immediately obtain the Fefferman-Stein vector-valued inequalities for the Doob maximal operator $M$ on $L^{p}(\Omega, w d \mathbb{P})$. This finishes the proof of Theorem 3.11 .

Now, we are in a position to prove the following Fefferman-Stein vector-valued Doob maximal inequality on Musielak-Orlicz spaces.

Theorem 3.12. Let $\varphi \in A_{\infty}(\Omega)$ be a Musielak-Orlicz function with uniformly lower type $p_{\varphi}^{-}$and uniformly upper type $p_{\varphi}^{+}$satisfying (3.2). Then, for any given $r \in(1, \infty)$, there exists a positive constant $C$ such that, for any sequence $\left\{f_{j}\right\}_{j \in \mathbb{N}}$ of measurable functions,

$$
\left\|\left\{\sum_{j \in \mathbb{N}}\left[M\left(f_{j}\right)\right]^{r}\right\}^{\frac{1}{r}}\right\|_{L^{\varphi}(\Omega)} \leq C\left\|\left(\sum_{j \in \mathbb{N}}\left|f_{j}\right|^{r}\right)^{\frac{1}{r}}\right\|_{L^{\varphi}(\Omega)} .
$$


Proof. Let $r \in(1, \infty)$. For any $j \in \mathbb{N}$ and $x \in \Omega$, if $\sum_{j \in \mathbb{N}}\left|f_{j}(x)\right|^{r} \neq 0$, let

$$
\widetilde{f}_{j}(x):=\frac{f_{j}(x)}{\left[\sum_{j \in \mathbb{N}}\left|f_{j}(x)\right|^{r}\right]^{\frac{1}{r}}}
$$

and, if $\sum_{j \in \mathbb{N}}\left|f_{j}(x)\right|^{r}=0, \widetilde{f}_{j}(x):=0$. Then, for any given $r \in(1, \infty)$ and any $x \in \Omega$, we obtain

$$
\sum_{j \in \mathbb{N}}\left|\widetilde{f}_{j}(x)\right|^{r}= \begin{cases}1 & \text { if } \sum_{j \in \mathbb{N}}\left|f_{j}(x)\right|^{r} \neq 0, \\ 0 & \text { if } \sum_{j \in \mathbb{N}}\left|f_{j}(x)\right|^{r}=0 .\end{cases}
$$

Since $q(\varphi)<p_{\varphi}^{-} \leq p_{\varphi}^{+}$, we can choose $p_{1} \in\left(q(\varphi), p_{\varphi}^{-}\right)$and $p_{2} \in\left[p_{\varphi}^{+}, \infty\right)$. For any given $r \in(1, \infty)$, consider the operator

$$
T(h):=\left\{\sum_{j \in \mathbb{N}}\left[M\left(h \widetilde{f}_{j}\right)\right]^{r}\right\}^{\frac{1}{r}},
$$

where $h \in L^{p_{1}}(\Omega, \varphi(\cdot, 1) d \mathbb{P})+L^{p_{2}}(\Omega, \varphi(\cdot, 1) d \mathbb{P})$. Then, from the sublinearity of $M$ and the Minkowski inequality, we deduce that $T$ is a sublinear operator. By (3.7) and Theorem 3.11, we find that, for any $i \in\{1,2\}, \alpha \in(0, \infty), t \in(0, \infty)$ and $h \in L^{p_{1}}(\Omega, \varphi(\cdot, 1) d \mathbb{P})+L^{p_{2}}(\Omega, \varphi(\cdot, 1) d \mathbb{P})$,

$$
\begin{aligned}
\varphi(\{x \in \Omega:|T(h)(x)|>\alpha\}, t) & =\varphi\left(\left\{x \in \Omega:\left\{\sum_{j \in \mathbb{N}}\left[M\left(h \widetilde{f}_{j}\right)(x)\right]^{r}\right\}^{\frac{1}{r}}>\alpha\right\}, t\right) \\
& \lesssim \alpha^{-p_{i}} \int_{\Omega}|h(x)|^{p_{i}}\left[\sum_{j \in \mathbb{N}}\left|\widetilde{f}_{j}(x)\right|^{r}\right]^{\frac{p_{i}}{r}} \varphi(x, t) d \mathbb{P}(x) \\
& \lesssim \alpha^{-p_{i}} \int_{\Omega}|h(x)|^{p_{i}} \varphi(x, t) d \mathbb{P}(x) .
\end{aligned}
$$

Thus, $T$ satisfies (3.1).

For any given $r \in(1, \infty)$ and any sequence $\left\{f_{j}\right\}_{j \in \mathbb{N}}$ of measurable functions, let

$$
h_{r}:=\left(\sum_{j \in \mathbb{N}}\left|f_{j}\right|^{r}\right)^{\frac{1}{r}} .
$$

Then, for any $j \in \mathbb{N}, h_{r} \widetilde{f}_{j}=f_{j}$. Combining this, (3.8) and Theorem 3.1, we conclude that, for any given $r \in(1, \infty)$,

$$
\int_{\Omega} \varphi\left(x, T\left(h_{r}\right)(x)\right) d \mathbb{P}(x) \lesssim \int_{\Omega} \varphi\left(x, h_{r}(x)\right) d \mathbb{P}(x),
$$

which implies that (3.6) holds true with some positive constant $C$. This finishes the proof of Theorem 3.12. 
Remark 3.13. Theorem 3.12 is a probabilistic version of [15, Corollary 6.1]. It should be noticed that [15, Corollary 6.1] does not capture weighted cases. This is because the assumptions of [15, Corollary 6.1] requires the weight to be essentially constant; see [15, p. 4331] for more details. However, Theorem 3.12 can cover this important case. To be precise, in Theorem 3.12, if we choose $\varphi(x, t):=w(x) \Phi(t)$ or $\varphi(x, t):=t^{p}+w(x) t^{q}$ for any $x \in \Omega$ and $t \in(0, \infty)$, where $1<p \leq$ $q<\infty, w$ is a special weight and $\Phi$ is an Orlicz function, then, by Example 2.16, we know that Theorem 3.12 cover the weighted Orlicz case and the case of the double phase functional, which are also new.

Theorem 3.12 also implies that the following Musielak-Orlicz version of the Stein inequality (see [71, p. 103, Theorem 8]) holds true.

Theorem 3.14. Let $\varphi \in A_{\infty}(\Omega)$ be a Musielak-Orlicz function with uniformly lower type $p_{\varphi}^{-}$and uniformly upper type $p_{\varphi}^{+}$satisfying (3.2). Then, for any given $r \in(1, \infty)$, there exists a positive constant $C$ such that, for any sequence $\left(g_{k}\right)_{k \in \mathbb{N}}$ of non-negative $\mathcal{F}$ measurable functions,

$$
\left\|\left\{\sum_{k \in \mathbb{N}}\left[\mathbb{E}_{k}\left(g_{k}\right)\right]^{r}\right\}^{\frac{1}{r}}\right\|_{L^{\varphi}(\Omega)} \leq C\left\|\left[\sum_{k \in \mathbb{N}}\left(g_{k}\right)^{r}\right]^{\frac{1}{r}}\right\|_{L^{\varphi}(\Omega)} .
$$

Proof. Notice that, for any $k \in \mathbb{N}, \mathbb{E}_{k}\left(g_{k}\right) \leq M\left(g_{k}\right)$. Then the desired conclusion follows immediately from Theorem 3.12. This finishes the proof of Theorem 3.14.

Remark 3.15. For any given $p \in(1, \infty)$, when $\varphi(x, t):=t^{p}$ for any $x \in \Omega$ and $t \in(0, \infty)$, Theorem 3.14 with $r=2$ was studied by Stein [71, p. 103, Theorem 8], and then Theorem 3.14 with $r \in(1, \infty)$ was investigated by Dilworth [18, Theorem 3.2 and Corollary 2.4]. If, in Theorem 3.14 , we choose $\varphi(x, t):=t$ for any $x \in \Omega$ and $t \in(0, \infty)$, then Theorem 3.14 with $r \in(1, \infty)$ breaks down (see [18, Remark 2.7]). Thus, since $\varphi$ in Theorem 3.14 is of wide generality, Theorem 3.14 generalize the Stein inequality to more general case. Especially, let $p \in(1, \infty)$ and $w$ be a weight, if $\varphi(x, t):=w(x) t^{p}$ for any $x \in \Omega$ and $t \in(0, \infty)$, then Theorem 3.14 becomes the weighted Stein inequality, which is also new.

\section{Atomic characterizations}

In this section, we establish the atomic characterization of martingale Musielak-Orlicz Hardy spaces $H_{\varphi}^{s}(\Omega), P_{\varphi}(\Omega), Q_{\varphi}(\Omega), H_{\varphi}^{M}(\Omega)$ and $H_{\varphi}^{S}(\Omega)$.

Theorem 4.1. Let $\varphi$ be a Musielak-Orlicz function. Then, for any given $r \in(0,1]$,

$$
H_{\varphi}^{s}(\Omega)=H_{\mathrm{at}, r}^{\varphi, \infty}(\Omega) \quad \text { with equivalent quasi-norms } .
$$

Proof. We prove this theorem by two steps.

Step 1) Prove $H_{\varphi}^{s}(\Omega) \subset H_{\text {at, } r}^{\varphi, s}(\Omega)$. To prove this, let $f \in H_{\varphi}^{s}(\Omega)$. For any $k \in \mathbb{Z}$, the stopping time $v^{k}$ is defined by setting

$$
v^{k}:=\inf \left\{n \in \mathbb{Z}_{+}: s_{n+1}(f)>2^{k}\right\} \quad(\inf \emptyset=\infty) .
$$


Obviously, the sequence $\left(v^{k}\right)_{k \in \mathbb{Z}}$ of stopping times is non-decreasing. Similarly to the proof of [77, Theorem 2.2], we have, for the stopped martingale $f^{\nu^{k}}:=\left(f_{n}^{v^{k}}\right)_{n \in \mathbb{Z}_{+}}:=\left(f_{n \wedge v^{k}}\right)_{n \in \mathbb{Z}_{+}}$,

$$
\sum_{k \in \mathbb{Z}}\left(f_{n}^{v^{k+1}}-f_{n}^{v^{k}}\right)=f_{n} \quad \text { almost everywhere. }
$$

For any $k \in \mathbb{Z}$, let

$$
\mu^{k}:=2^{k+1}\left\|\mathbf{1}_{\left\{v^{k}<\infty\right\}}\right\|_{L^{\varphi}(\Omega)} .
$$

Moreover, for any $k \in \mathbb{Z}$ and $n \in \mathbb{Z}_{+}$, if $\mu^{k} \neq 0$, let

$$
a_{n}^{k}:=\frac{f_{n}^{v^{k+1}}-f_{n}^{v^{k}}}{\mu^{k}}
$$

otherwise, let $a_{n}^{k}:=0$. We first show that, for any fixed $k \in \mathbb{Z}, a^{k}:=\left(a_{n}^{k}\right)_{n \in \mathbb{Z}_{+}}$is a $(\varphi, \infty)_{s}$-atom. By the definition of $a^{k}$, it is easy to know that $a^{k}$ is a martingale. Then, for any $n \in \mathbb{Z}_{+}$, when $v^{k} \geq n$, by the definition of $f^{v^{k}}$, we have

$$
a_{n}^{k}=\frac{f_{n}^{v^{k+1}}-f_{n}^{v^{k}}}{\mu^{k}}=\frac{f_{n}-f_{n}}{\mu^{k}}=0 .
$$

Thus, $a^{k}$ satisfies Definition 2.3(i). From (4.1), we further deduce that

$$
\mathbf{1}_{\left\{v^{k}=\infty\right\}}\left[s\left(a^{k}\right)\right]^{2} \leq \sum_{n=1}^{\infty} \mathbf{1}_{\left\{v^{k} \geq n\right\}} \mathbb{E}_{n-1}\left(\left|d_{n} a^{k}\right|^{2}\right)=\sum_{n=1}^{\infty} \mathbb{E}_{n-1}\left(\left|\mathbf{1}_{\left\{v^{k} \geq n\right\}} d_{n} a^{k}\right|^{2}\right)=0 .
$$

Thus, we have

$$
\left\{x \in \Omega: s\left(a^{k}\right)(x) \neq 0\right\} \subset B_{v^{k}} .
$$

Moreover, by the definition of $v^{k}$, we obtain

$$
\left[s\left(a^{k}\right)\right]^{2}=\sum_{n=1}^{\infty} \mathbb{E}_{n-1}\left(\left|d_{n} a^{k}\right|^{2}\right) \leq\left[\frac{s_{v^{k+1}}(f)}{\mu^{k}}\right]^{2} \leq\left(\frac{2^{k+1}}{\mu^{k}}\right)^{2},
$$

which implies that $\left(a_{n}^{k}\right)_{n \in \mathbb{Z}_{+}}$is an $L^{2}$-bounded martingale and hence $\left(a_{n}^{k}\right)_{n \in \mathbb{Z}_{+}}$converges in $L^{2}(\Omega)$. Denoting the limit still by $a^{k}$, then, for any $n \in \mathbb{Z}_{+}, \mathbb{E}_{n}\left(a^{k}\right)=a_{n}^{k}$. For any $n \in \mathbb{Z}_{+}$and $n \leq v^{k}$, we know that $a_{n}^{k}=0$ and $\left\|s\left(a^{k}\right)\right\|_{L^{\infty}(\Omega)} \leq \frac{2^{k+1}}{\mu^{k}}$. Therefore,

$$
\left\|s\left(a^{k}\right)\right\|_{L^{\infty}\left(B_{v^{k}}\right)} \leq \frac{2^{k+1}}{\mu^{k}}=\left\|\mathbf{1}_{B_{v^{k}}}\right\|_{L^{\varphi}(\Omega)}^{-1} .
$$

Thus, $a^{k}$ satisfies Definition 2.3(ii) and we further conclude that $a^{k}$ is a $(\varphi, \infty)_{s}$-atom.

We now show that $\left\{\mu^{k}, a^{k}, v^{k}\right\}_{k \in \mathbb{Z}} \in \mathcal{A}_{s}(\varphi, \infty)$. To this end, we first notice that

$$
\sum_{k \in \mathbb{Z}}\left[\frac{\mu^{k} \mathbf{1}_{B_{v^{k}}}}{\left\|\mathbf{1}_{B_{v^{k}}}\right\|_{L^{\varphi}(\Omega)}}\right]^{r}=\sum_{k \in \mathbb{Z}}\left(2^{k+1} \mathbf{1}_{B_{v^{k}}}\right)^{r} .
$$


For any $k \in \mathbb{Z}$, by the definition of $v^{k}$, we find that $B_{\gamma^{k}}=\left\{x \in \Omega: s(f)(x)>2^{k}\right\}$ and $B_{\gamma^{k}} \supset B_{\gamma^{k+1}}$. Let $G_{k}:=B_{v^{k}} \backslash B_{v^{k+1}}$, then $G_{k}:=\left\{x \in \Omega: 2^{k}<s(f)(x) \leq 2^{k+1}\right\}$. Now we claim that, for any given $r \in(0, \infty)$,

$$
\sum_{k \in \mathbb{Z}}\left(2^{k+1} \mathbf{1}_{B_{v^{k}}}\right)^{r}=\frac{2^{r}}{2^{r}-1}\left(\sum_{k \in \mathbb{Z}} 2^{k+1} \mathbf{1}_{G_{k}}\right)^{r} .
$$

Indeed, for any $k \in \mathbb{Z}, B_{v^{k}}=\bigcup_{j=k}^{\infty} G_{j}$. From this and the fact that $\left\{G_{k}\right\}_{k \in \mathbb{Z}}$ are disjoint, it follows that, for any $k \in \mathbb{Z}$,

$$
\mathbf{1}_{B_{v^{k}}}=\sum_{j=k}^{\infty} \mathbf{1}_{G_{j}},
$$

which, implies that, for any given $r \in(0, \infty)$,

$$
\sum_{k \in \mathbb{Z}}\left(2^{k+1} \mathbf{1}_{B_{\nu^{k}}}\right)^{r}=\sum_{k \in \mathbb{Z}} 2^{(k+1) r}\left(\sum_{j=k}^{\infty} \mathbf{1}_{G_{j}}\right)=\sum_{j \in \mathbb{Z}} \sum_{k \leq j} 2^{(k+1) r} \mathbf{1}_{G_{j}}=\frac{2^{r}}{2^{r}-1} \sum_{k \in \mathbb{Z}} 2^{(k+1) r} \mathbf{1}_{G_{k}} .
$$

Combining this and the fact that $\left\{G_{k}\right\}_{k \in \mathbb{Z}}$ are disjoint, we obtain (4.3). This proves the above claim. From this claim, the definition of $G_{k}$ and (4.2), we deduce that, for any given $r \in(0, \infty)$ and any $\lambda \in(0, \infty)$,

$$
\begin{aligned}
\int_{\Omega} \varphi\left(x, \frac{1}{\lambda}\left\{\sum_{k \in \mathbb{Z}}\left[\frac{\mu^{k} \mathbf{1}_{B_{\gamma^{k}}}(x)}{\left\|\mathbf{1}_{B_{v^{k}}}\right\|_{L^{\varphi}(\Omega)}}\right]^{r}\right\}^{\frac{1}{r}}\right) d \mathbb{P}(x) & =\int_{\Omega} \varphi\left(x, \frac{1}{\lambda}\left(\frac{2^{r}}{2^{r}-1}\right)^{\frac{1}{r}} \sum_{k \in \mathbb{Z}} 2^{k+1} \mathbf{1}_{G_{k}}(x)\right) d \mathbb{P}(x) \\
& \leq \sum_{k \in \mathbb{Z}} \int_{G_{k}} \varphi\left(x, 2\left(\frac{2^{r}}{2^{r}-1}\right)^{\frac{1}{r}} \frac{s(f)(x)}{\lambda}\right) d \mathbb{P}(x) \\
& \leq \int_{\Omega} \varphi\left(x, 2\left(\frac{2^{r}}{2^{r}-1}\right)^{\frac{1}{r}} \frac{s(f)(x)}{\lambda}\right) d \mathbb{P}(x) .
\end{aligned}
$$

For any given $r \in(0, \infty)$, letting $\lambda:=2\left(\frac{2^{r}}{2^{r}-1}\right)^{\frac{1}{r}}\|f\|_{H_{\varphi}^{s}(\Omega)}$, then we obtain

$$
\left\|\left\{\sum_{k \in \mathbb{Z}}\left[\frac{\mu^{k} \mathbf{1}_{B_{v^{k}}}(x)}{\left\|\mathbf{1}_{B_{\nu^{k}}}\right\|_{L^{\varphi}}(\Omega)}\right]^{r}\right\}^{\frac{1}{r}}\right\|_{L^{\varphi}(\Omega)} \leq 2\left(\frac{2^{r}}{2^{r}-1}\right)^{\frac{1}{r}}\|f\|_{H_{\varphi}^{s}(\Omega)}
$$

This implies that, for any given $r \in(0, \infty), f \in H_{\mathrm{at}, r}^{\varphi, \infty, s}(\Omega)$ and $\|f\|_{H_{\mathrm{at}, r}^{\varphi, \infty, s}(\Omega)} \lesssim\|f\|_{H_{\varphi}^{s}(\Omega)}$. The proof of Step 1) is now complete.

Step 2) Prove $H_{\mathrm{at}, r}^{\varphi, \infty, s}(\Omega) \subset H_{\varphi}^{s}(\Omega)$. To show this, for any given $r \in(0,1]$, let $f \in H_{\mathrm{at}, r}^{\varphi, \infty}(\Omega)$. Then we can write $f=\sum_{k \in \mathbb{Z}} \mu^{k} a^{k}$ for some sequence of triples,

$$
\left\{\mu^{k}, a^{k}, v^{k}\right\}_{k \in \mathbb{Z}} \in \mathcal{A}_{s}(\varphi, \infty) .
$$

By Definition 2.3(i), we know that, for any $k \in \mathbb{Z}$,

$$
\left\{x \in \Omega: s\left(a^{k}\right)(x) \neq 0\right\} \subset B_{v^{k}} .
$$


Thus, for almost every $x \in \Omega$, we have

$$
s\left(a^{k}\right)(x) \leq\left\|s\left(a^{k}\right)\right\|_{L^{\infty}(\Omega)} \mathbf{1}_{B_{v^{k}}}(x) \leq \mathbf{1}_{B_{v^{k}}}(x)\left\|\mathbf{1}_{B_{v^{k}}}\right\|_{L^{\varphi}(\Omega)}^{-1} .
$$

Combining this and the subadditive of the operator $s$, we find that, for any given $r \in(0,1]$ and $\lambda \in(0, \infty)$,

$$
\begin{aligned}
\int_{\Omega} \varphi\left(x, \frac{s(f)(x)}{\lambda}\right) d \mathbb{P}(x) & \leq \int_{\Omega} \varphi\left(x, \sum_{k \in \mathbb{Z}} \frac{\mu^{k} s\left(a^{k}\right)(x)}{\lambda}\right) d \mathbb{P}(x) \leq \int_{\Omega} \varphi\left(x, \sum_{k \in \mathbb{Z}} \frac{\mu^{k} \mathbf{1}_{B_{v^{k}}}(x)}{\lambda\left\|\mathbf{1}_{B_{v^{k}}}\right\|_{L^{\varphi}(\Omega)}}\right) d \mathbb{P}(x) \\
& \leq \int_{\Omega} \varphi\left(x, \frac{1}{\lambda}\left\{\sum_{k \in \mathbb{Z}}\left[\frac{\mu^{k} \mathbf{1}_{B_{v^{k}}}(x)}{\left\|\mathbf{1}_{B_{v^{k}}}\right\|_{L^{\varphi}(\Omega)}}\right]^{r}\right\}^{\frac{1}{r}}\right) d \mathbb{P}(x) .
\end{aligned}
$$

Therefore, for any given $r \in(0,1]$, we conclude that $f \in H_{\varphi}^{s}(\Omega)$ and $\|f\|_{H_{\varphi}^{s}(\Omega)} \leq\|f\|_{H_{\mathrm{at}, r}^{\varphi, \infty, s}(\Omega)}$. This finishes the proof of Theorem 4.1.

Remark 4.2. Clearly, Theorem 4.1 does not need the assumptions that $\varphi$ is of uniformly lower type $p$ for some $p \in(0,1]$ and of uniformly upper type 1 . This is different from [79, Theorem 1.4] which need the both assumptions.

Remark 4.3. If $\varphi$ is of uniformly upper type $p$ for some $p \in(0, \infty)$ and $\varphi(x, \cdot)$ is right-continuous at 0 for almost every $x \in \Omega$ in Theorem 4.1 , then the sum $\sum_{k=l}^{m} \mu^{k} a^{k}$ converges to $f$ in $H_{\varphi}^{s}(\Omega)$ as $m \rightarrow \infty, \ell \rightarrow-\infty$. Indeed, for any $m, \ell \in \mathbb{Z}$ and $\ell<m$, we obtain

$$
f-\sum_{k=l}^{m} \mu^{k} a^{k}=\left(f-f^{\nu^{m+1}}\right)+f^{\nu^{l}} \quad \text { and } \quad\left[s\left(f-f^{\nu^{m+1}}\right)\right]^{2}=[s(f)]^{2}-\left[s\left(f^{\nu^{m+1}}\right)\right]^{2} .
$$

From this, we deduce that, for any $m, \ell \in \mathbb{Z}$ with $\ell<m$ and $x \in \Omega$,

$$
\begin{aligned}
\varphi\left(x, s\left(f-\sum_{k=l}^{m} \mu^{k} a^{k}\right)(x)\right) & \leq \varphi\left(x, s\left(f-f^{v^{m+1}}\right)(x)+s\left(f^{v^{l}}\right)(x)\right) \\
& \leq \varphi\left(x, 2 s\left(f-f^{v^{m+1}}\right)(x)\right)+\varphi\left(x, 2 s\left(f^{\nu^{l}}\right)(x)\right) .
\end{aligned}
$$

In addition, for any $m, \ell \in \mathbb{Z}$ and almost every $x \in \Omega$,

$$
\lim _{m \rightarrow \infty} s\left(f-f^{v^{m+1}}\right)(x)=0 \text { and } \quad \lim _{l \rightarrow-\infty} s\left(f^{\nu^{l}}\right)(x)=0 .
$$

Combining this and $\varphi(x, \cdot)$ is right-continuous at 0 for almost every $x \in \Omega$, we conclude that, for any $m, \ell \in \mathbb{Z}$ and almost every $x \in \Omega$,

$$
\lim _{m \rightarrow \infty} \varphi\left(x, 2 s\left(f-f^{v^{m+1}}\right)(x)\right)=0 \quad \text { and } \quad \lim _{l \rightarrow-\infty} \varphi\left(x, 2 s\left(f^{v^{l}}\right)(x)\right)=0 .
$$

Also, we find that, for any $m, \ell \in \mathbb{Z}$,

$$
s\left(f-f^{v^{m+1}}\right) \leq s(f) \quad \text { and } \quad s\left(f^{\nu^{l}}\right) \leq s(f),
$$

which, together with (4.5), (4.6), the fact that $\varphi$ is of uniformly upper type $p$ for some $p \in(0, \infty)$ and the Lebesgue dominated convergence theorem, implies that the series $\sum_{k=l}^{m} \mu^{k} a^{k}$ converges to $f$ in $H_{\varphi}^{s}(\Omega)$ norm as $m \rightarrow \infty, l \rightarrow-\infty$. 
Similarly, we also obtain the following atomic characterizations of $P_{\varphi}(\Omega)$ and $Q_{\varphi}(\Omega)$.

Theorem 4.4. Let $\varphi$ be a Musielak-Orlicz function. Then, for any given $r \in(0,1]$,

$$
P_{\varphi}(\Omega)=H_{\mathrm{at}, r}^{\varphi, \infty}(\Omega) \quad \text { and } \quad Q_{\varphi}(\Omega)=H_{\mathrm{at}, r}^{\varphi, \infty}(\Omega) \quad \text { with equivalent quasi-norms. }
$$

Proof. The proof of Theorem 4.4 is similar to that of Theorem 4.1. So we just give the outline and omit the details. We only give the proof for $P_{\varphi}(\Omega)$ because it is just slightly different from the one for $Q_{\varphi}(\Omega)$.

Let $f \in P_{\varphi}(\Omega)$ and $\left(\lambda_{n}\right)_{n \in \mathbb{N}}$ be an adapted non-decreasing sequence satisfying that, for any $n \in \mathbb{N},\left|f_{n}\right| \leq \lambda_{n-1}$ and $\lambda_{\infty} \in L^{\varphi}(\Omega)$. The stopping times, in these cases, are defined by setting, for any $k \in \mathbb{Z}$,

$$
v^{k}:=\inf \left\{n \in \mathbb{N}: \lambda_{n}>2^{k}\right\} \quad(\inf \emptyset=\infty) .
$$

For any $k \in \mathbb{Z}$, let $a^{k}$ and $\mu^{k}$ be defined as in the proof of Theorem 4.1. Then, similarly to the proof of Theorem 4.1, we easily know that $a^{k}$ is a $(\varphi, \infty)_{M}$-atom. By the argument same as in the proof of Theorem 4.1, we can prove that, for any given $r \in(0,1]$,

$$
\left\|\left\{\sum_{k \in \mathbb{Z}}\left[\frac{\mu^{k} \mathbf{1}_{B_{v^{k}}}(x)}{\left\|\mathbf{1}_{B_{v^{k}}}\right\|_{L^{\varphi}(\Omega)}}\right]^{r}\right\}^{\frac{1}{r}}\right\|_{L^{\varphi}(\Omega)} \leq 2\left(\frac{2^{r}}{2^{r}-1}\right)^{\frac{1}{r}}\left\|\lambda_{\infty}\right\|_{L^{\varphi}(\Omega)} .
$$

This implies that, for any given $r \in(0,1],\|f\|_{H_{\mathrm{at}, r}^{\varphi, \infty}(\Omega)} \lesssim\|f\|_{P_{\varphi}(\Omega)}$ and $P_{\varphi}(\Omega) \subset H_{\mathrm{at}, r}^{\varphi, \infty}(\Omega)$.

Conversely, for any given $r \in(0,1]$, assume that $f \in H_{\mathrm{at}, r}^{\varphi, \infty, M}(\Omega)$. Then we can write $f=$ $\sum_{k \in \mathbb{Z}} \mu^{k} a^{k}$ for some sequence of triples,

$$
\left\{\mu^{k}, a^{k}, v^{k}\right\}_{k \in \mathbb{Z}} \in \mathcal{A}_{M}(\varphi, \infty)
$$

For any $n \in \mathbb{Z}_{+}$, let

$$
\lambda_{n}:=\sum_{k \in \mathbb{Z}} \mu^{k} \mathbf{1}_{\left\{x \in \Omega: v^{k}(x) \leq n\right\}}\left\|M\left(a^{k}\right)\right\|_{L^{\infty}(\Omega)} .
$$

Then $\left\{\lambda_{n}\right\}_{n \in \mathbb{Z}_{+}}$is a nonnegative adapted sequence and, for any $n \in \mathbb{Z}_{+},\left|f_{n}\right| \leq \lambda_{n-1}$. Thus, for any given $r \in(0,1]$, we have

$$
\|f\|_{P_{\varphi}(\Omega)} \leq\left\|\lambda_{\infty}\right\|_{L^{\varphi}(\Omega)} \leq\left\|\sum_{k \in \mathbb{Z}} \frac{\mu^{k} \mathbf{1}_{B_{\nu^{k}}}}{\left\|\mathbf{1}_{B_{\nu^{k}}}\right\|_{L^{\varphi}(\Omega)}}\right\|_{L^{\varphi}(\Omega)} \leq\left\|\left\{\sum_{k \in \mathbb{Z}}\left[\frac{\mu^{k} \mathbf{1}_{B_{\nu^{k}}}(x)}{\left\|\mathbf{1}_{B_{v^{k}}}\right\|_{L^{\varphi}}(\Omega)}\right]^{r}\right\}^{\frac{1}{r}}\right\|_{L^{\varphi}(\Omega)} .
$$

From this, it follows that, for any given $r \in(0,1], f \in P_{\varphi}(\Omega)$ and $\|f\|_{P_{\varphi}(\Omega)} \leq\|f\|_{H_{\mathrm{at}, r}^{\varphi, \infty, M}(\Omega)}$. This finishes the proof of Theorem 4.4.

Remark 4.5. If $\varphi$ is of uniformly upper type $p$ for some $p \in(0, \infty)$ and $\varphi(x, \cdot)$ is right-continuous at 0 for almost every $x \in \Omega$, then we claim that the sum $\sum_{k=l}^{m} \mu^{k} a^{k}$ converges to $f$ in $P_{\varphi}(\Omega)$ as $l \rightarrow-\infty$ and $m \rightarrow \infty$. Indeed, for any $n \in \mathbb{N}$ and $\ell, m \in \mathbb{Z}$, let

$$
\lambda_{n, 1}:=\sum_{k=m+1}^{\infty} \mu^{k} \mathbf{1}_{\left\{x \in \Omega: v^{k}(x) \leq n\right\}}\left\|M\left(a^{k}\right)\right\|_{L^{\infty}(\Omega)} \quad \text { and } \quad \lambda_{n, 2}:=\sum_{k=-\infty}^{\ell} \mu^{k} \mathbf{1}_{\left\{x \in \Omega: v^{k}(x) \leq n\right\}}\left\|M\left(a^{k}\right)\right\|_{L^{\infty}(\Omega)} .
$$


Then $\left\{\lambda_{n, 1}\right\}_{n \in \mathbb{N}}$ and $\left\{\lambda_{n, 2}\right\}_{n \in \mathbb{N}}$ are nonnegative adapted sequences and, for any $n \in \mathbb{N}$ and $\ell, m \in \mathbb{Z}$,

$$
\begin{aligned}
\left|f_{n}-\sum_{k=l}^{m} \mu^{k} a_{n}^{k}\right| & =\left|f_{n}-f_{n}^{\nu^{m+1}}+f_{n}^{\nu^{\ell}}\right| \leq \sum_{k=m+1}^{\infty}\left|f_{n}^{v^{k+1}}-f_{n}^{\nu^{k}}\right|+\sum_{k=-\infty}^{\ell}\left|f_{n}^{v^{k+1}}-f_{n}^{v^{k}}\right| \\
& =\sum_{k=m+1}^{\infty} \mu^{k} \mathbf{1}_{\left\{x \in \Omega: \nu^{k}(x) \leq n-1\right\}}\left|a_{n}^{k}\right|+\sum_{k=-\infty}^{\ell} \mu^{k} \mathbf{1}_{\left\{x \in \Omega: \nu^{k}(x) \leq n-1\right\}}\left|a_{n}^{k}\right| \leq \lambda_{n-1,1}+\lambda_{n-1,2} .
\end{aligned}
$$

Using this, the analogue of (4.5) and (4.6), we obtain

$$
\left\|f-\sum_{k=l}^{m} \mu^{k} a^{k}\right\|_{P_{\varphi}(\Omega)} \rightarrow 0 \quad \text { as } l \rightarrow-\infty \text { and } m \rightarrow \infty,
$$

which completes the proof of the above claim.

The following atomic characterizations of Musielak-Orlicz Hardy spaces $H_{\varphi}^{M}(\Omega)$ and $H_{\varphi}^{S}(\Omega)$ are new even for classical martingale Hardy spaces. For the dyadic stochastic basis, it was proved by Weisz [78].

Theorem 4.6. Let $\varphi$ be a Musielak-Orlicz function and of uniformly lower type $p \in(0, \infty)$. If $\varphi \in \mathbb{S}^{-}$and the stochastic basis is regular, then, for any given $r \in(0,1]$,

$$
H_{\varphi}^{M}(\Omega)=H_{\mathrm{at}, r}^{\varphi, \infty}(\Omega) \quad \text { and } \quad H_{\varphi}^{S}(\Omega)=H_{\mathrm{at}, r}^{\varphi, \infty}(\Omega) \quad \text { with equivalent quasi-norms. }
$$

To show Theorem 4.6, we need the next technical lemma, which might have some independent interest.

Lemma 4.7. Let $w$ be a special weight and $w:=\left(w_{n}\right)_{n \in \mathbb{Z}_{+}} \in \mathbb{S}^{-}$. If the stochastic basis $\left\{\mathcal{F}_{n}\right\}_{n \in \mathbb{Z}_{+}}$ is regular, then, for any given nonnegative adapted process $\gamma=\left(\gamma_{n}\right)_{n \in \mathbb{Z}_{+}}$and $\lambda \in\left(\left\|\gamma_{0}\right\|_{L^{\infty}(\Omega)}, \infty\right)$, there exists a stopping time $\tau_{\lambda}$ such that

$$
\begin{gathered}
\{x \in \Omega: M(\gamma)(x)>\lambda\} \subset\left\{x \in \Omega: \tau_{\lambda}(x)<\infty\right\}, \\
\sup _{n \leq \tau_{\lambda}(x)} \gamma_{n}(x)=: M_{\tau_{\lambda}}(\gamma)(x) \leq \lambda, \quad \forall x \in \Omega
\end{gathered}
$$

and

$$
w\left(\left\{x \in \Omega: \tau_{\lambda}(x)<\infty\right\}\right) \leq R K w(\{x \in \Omega: M(\gamma)(x)>\lambda\}),
$$

where $R$ and $K$ are the same as in (2.5) and (2.4), respectively. Moreover, for any $\lambda_{1}, \lambda_{2} \in(0, \infty)$ with $\lambda_{1}<\lambda_{2}, \tau_{\lambda_{1}} \leq \tau_{\lambda_{2}}$.

Proof. Let $\lambda \in\left(\left\|\gamma_{0}\right\|_{L^{\infty}(\Omega)}, \infty\right)$ and $\gamma=\left(\gamma_{n}\right)_{n \in \mathbb{Z}_{+}}$be any nonnegative adapted process. For any $n \in \mathbb{N}$, let

$$
G_{n}:=\left\{x \in \Omega: \frac{1}{w_{n-1}(x)} \mathbb{E}\left(\mathbf{1}_{\left\{x \in \Omega: \gamma_{n}(x)>\lambda\right\}} w \mid \mathcal{F}_{n-1}\right)(x)>\frac{1}{R K}\right\}
$$


and

$$
\tau_{\lambda}(x):=\inf \left\{n \in \mathbb{Z}_{+}: x \in G_{n+1}\right\}, \quad \forall x \in \Omega .
$$

Clearly, for any $n \in \mathbb{N}, G_{n} \in \mathcal{F}_{n-1}$ and hence $\tau_{\lambda}$ is a stopping time. We claim that (4.8) holds true. Indeed, by $w \in \mathbb{S}^{-}$, the regularity and the fact that $\left(\gamma_{n}\right)_{n \in \mathbb{Z}_{+}}$is adapted, we find that, for any $n \in \mathbb{N}$,

$$
\mathbf{1}_{\left\{x \in \Omega: \gamma_{n}(x)>\lambda\right\}}=\frac{1}{w_{n}} \mathbb{E}\left(\mathbf{1}_{\left\{x \in \Omega: \gamma_{n}(x)>\lambda\right\}} w \mid \mathcal{F}_{n}\right) \leq \frac{K}{w_{n-1}} R \mathbb{E}\left(\mathbf{1}_{\left\{x \in \Omega: \gamma_{n}(x)>\lambda\right\}} w \mid \mathcal{F}_{n-1}\right) .
$$

From this, it follows that, for any $n \in \mathbb{N}$,

$$
\left\{x \in \Omega: \gamma_{n}(x)>\lambda\right\} \subset G_{n} .
$$

Since $\lambda>\left\|\gamma_{0}\right\|_{L^{\infty}(\Omega)}$, it follows that, for any $x \in\{x \in \Omega: M(\gamma)(x)>\lambda\}$, there exists $n \in \mathbb{Z}_{+}$such that

$$
x \in\left\{x \in \Omega: \gamma_{n+1}(x)>\lambda\right\} .
$$

Combining this and (4.11), we obtain $x \in G_{n+1}$. This implies that $\tau_{\lambda}(x) \leq n$, and hence (4.8) holds true. This proves the above claim.

Now we show (4.9). When $x \in\left\{x \in \Omega: \tau_{\lambda}(x)=0\right\}$, (4.9) follows from the assumption $\lambda>\left\|\gamma_{0}\right\|_{L^{\infty}(\Omega)}$. When $x \in\left\{x \in \Omega: \tau_{\lambda}(x)=\infty\right\}$, by (4.8), we know that

$$
\left\{x \in \Omega: \tau_{\lambda}(x)=\infty\right\} \subset\{x \in \Omega: M(\gamma)(x) \leq \lambda\} .
$$

In the remaining case $x \in\left\{x \in \Omega: 0<\tau_{\lambda}(x)<\infty\right\}$, there exists a positive integer $n$ such that $\tau_{\lambda}(x)=n$. From the definition of $\tau_{\lambda}$, it follows that $x \notin \bigcup_{m=1}^{n} G_{m}$. Combining this and (4.11), we obtain

$$
x \in \bigcup_{m=1}^{n}\left\{x \in \Omega: \gamma_{n}(x) \leq \lambda\right\} .
$$

This implies that $M_{\tau_{\lambda}}(\gamma)(x) \leq \lambda$. Therefore, (4.9) holds true.

Next we prove (4.10). From the definition of $\tau_{\lambda}$ and $\left\{x \in \Omega: \tau_{\lambda}(x)=n\right\} \in \mathcal{F}_{n}$, it follows that, for any $n \in \mathbb{Z}_{+}$,

$$
\begin{aligned}
\left\{x \in \Omega: \tau_{\lambda}(x)=n\right\} & =\left\{x \in \Omega: \tau_{\lambda}(x)=n\right\} \cap G_{n+1} \\
& =\left\{x \in \Omega: \frac{1}{w_{n}(x)} \mathbb{E}\left(\mathbf{1}_{\left\{x \in \Omega: \gamma_{n+1}(x)>\lambda\right\}} w \mid \mathcal{F}_{n}\right)(x) \mathbf{1}_{\left\{x \in \Omega: \tau_{\lambda}(x)=n\right\}}(x)>\frac{1}{R K}\right\} \\
& =\left\{x \in \Omega: \frac{1}{w_{n}(x)} \mathbb{E}\left(\mathbf{1}_{\left\{x \in \Omega: \gamma_{n+1}(x)>\lambda\right\} \cap\left\{x \in \Omega: \tau_{\lambda}(x)=n\right\}} w \mid \mathcal{F}_{n}\right)(x)>\frac{1}{R K}\right\},
\end{aligned}
$$

which, together with (4.8) and the fact that, for any $n, m \in \mathbb{Z}_{+}$and $n \neq m$,

$$
\left\{x \in \Omega: \tau_{\lambda}(x)=n\right\} \cap\left\{x \in \Omega: \tau_{\lambda}(x)=m\right\}=\varnothing,
$$

implies that

$$
w\left(\left\{x \in \Omega: \tau_{\lambda}(x)<\infty\right\}\right)=\sum_{n \in \mathbb{Z}_{+}} w\left(\left\{x \in \Omega: \tau_{\lambda}(x)=n\right\}\right)
$$




$$
\begin{aligned}
& \leq \sum_{n \in \mathbb{Z}_{+}} R K \int_{\Omega} \frac{1}{w_{n}(x)} \mathbb{E}\left(\mathbf{1}_{\left\{x \in \Omega: \gamma_{n+1}(x)>\lambda\right\} \cap\left\{x \in \Omega: \tau_{\lambda}(x)=n\right\}} w \mid \mathcal{F}_{n}\right)(x) w(x) d \mathbb{P}(x) \\
& =\sum_{n \in \mathbb{Z}_{+}} R K \int_{\Omega} \frac{1}{w_{n}(x)} \mathbb{E}\left(\mathbf{1}_{\left\{x \in \Omega: \gamma_{n+1}(x)>\lambda\right\} \cap\left\{x \in \Omega: \tau_{\lambda}(x)=n\right\}} w \mid \mathcal{F}_{n}\right)(x) w_{n}(x) d \mathbb{P}(x) \\
& =\sum_{n \in \mathbb{Z}_{+}} R K w\left(\left\{x \in \Omega: \gamma_{n+1}(x)>\lambda\right\} \cap\left\{x \in \Omega: \tau_{\lambda}(x)=n\right\}\right) \\
& \leq \sum_{n \in \mathbb{Z}_{+}} R K w\left(\{x \in \Omega: M(\gamma)(x)>\lambda\} \cap\left\{x \in \Omega: \tau_{\lambda}(x)=n\right\}\right) \\
& =R K w(\{x \in \Omega: M(\gamma)(x)>\lambda\}) .
\end{aligned}
$$

Thus, (4.10) holds true.

Finally, for any $\lambda_{1}, \lambda_{2} \in(0, \infty)$ with $\lambda_{1}<\lambda_{2}$, we know that, for any $n \in \mathbb{N}$,

$$
\mathbb{E}\left(\mathbf{1}_{\left\{x \in \Omega: \gamma_{n}(x)>\lambda_{1}\right\}} w \mid \mathcal{F}_{n-1}\right) \geq \mathbb{E}\left(\mathbf{1}_{\left\{x \in \Omega: \gamma_{n}(x)>\lambda_{2}\right\}} w \mid \mathcal{F}_{n-1}\right) .
$$

Combining this and the definitions of $G_{n}$ and $\tau_{\lambda}$, we obtain $\tau_{\lambda_{1}} \leq \tau_{\lambda_{2}}$. This finishes the proof of Lemma 4.7.

Now we are ready to prove Theorem 4.6.

Proof of Theorem 4.6. We prove this theorem only for $H_{\varphi}^{M}(\Omega)$ because the proof for $H_{\varphi}^{S}(\Omega)$ is similar. We do this by two steps.

Step 1) Prove $H_{\varphi}^{M}(\Omega) \subset H_{\text {at, } r}^{\varphi, \infty}(\Omega)$. To this end, let $f:=\left(f_{n}\right)_{n \in \mathbb{Z}_{+}} \in H_{\varphi}^{M}(\Omega)$. For any $k \in \mathbb{Z}$ and nonnegative adapted sequence $\left(\left|f_{n}\right|\right)_{n \in \mathbb{Z}_{+}}$, by Lemma 4.7, we know that there exists a stopping time $v^{k}$ such that

$$
\begin{gathered}
\left\{x \in \Omega: M(f)(x)>2^{k}\right\} \subset\left\{x \in \Omega: v^{k}(x)<\infty\right\}=: B_{v^{k}}, \\
M_{v^{k}}(f)(x) \leq 2^{k}, \quad \forall x \in \Omega
\end{gathered}
$$

and

$$
\varphi\left(\left\{x \in \Omega: v^{k}(x)<\infty\right\}, t\right) \leq R K \varphi\left(\left\{x \in \Omega: M(f)(x)>2^{k}\right\}, t\right), \quad \forall t \in(0, \infty) .
$$

Moreover, the sequence $\left\{v^{k}\right\}_{k \in \mathbb{Z}}$ of stopping times is non-decreasing and $v^{k} \rightarrow \infty$ as $k \rightarrow \infty$. Similarly to the proof of Theorem 4.1 , for any $k \in \mathbb{Z}$, let

$$
\mu^{k}:=3 \times 2^{k}\left\|\mathbf{1}_{B_{v^{k}}}\right\|_{L^{\varphi}(\Omega)} .
$$

Moreover, for any $k \in \mathbb{Z}$ and $n \in \mathbb{Z}_{+}$, if $\mu^{k} \neq 0$, let

$$
a_{n}^{k}:=\frac{f_{n}^{v^{k+1}}-f_{n}^{v^{k}}}{\mu^{k}} ;
$$




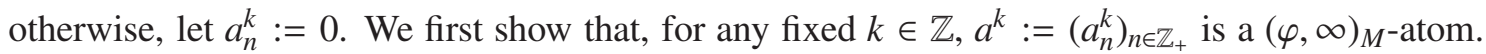
Clearly, $a^{k}$ is a martingale and

$$
\left\{x \in \Omega: M\left(a^{k}\right)(x) \neq 0\right\} \subset B_{v^{k}} .
$$

By (4.12), we have, for any $n \in \mathbb{Z}_{+}$,

$$
\left|a_{n}^{k}\right| \leq \frac{\left|f_{n}^{k^{k+1}}\right|+\left|f_{n}^{v^{k}}\right|}{\mu^{k}} \leq \frac{1}{\mu^{k}}\left[M_{\nu^{k+1}}(f)+M_{\nu^{k}}(f)\right] \leq\left\|\mathbf{1}_{B_{\nu^{k}}}\right\|_{L^{\varphi}(\Omega)}^{-1},
$$

which implies that

$$
\left\|M\left(a^{k}\right)\right\|_{L^{\infty}\left(B_{\gamma^{k}}\right)} \leq\left\|\mathbf{1}_{B_{v^{k}}}\right\|_{L^{\varphi}(\Omega)}^{-1} .
$$

Thus, for any $p \in(1, \infty),\left(a_{n}^{k}\right)_{n \in \mathbb{Z}_{+}}$is an $L^{p}$-bounded martingale and hence $\left(a_{n}^{k}\right)_{n \in \mathbb{Z}_{+}}$converges in $L^{p}(\Omega)$. Denoting this limit still by $a^{k}$, then $\mathbb{E}_{n}\left(a^{k}\right)=a_{n}^{k}$. For any $n \in \mathbb{Z}_{+}$, if $v^{k} \geq n$, by the definition of $f^{v^{k}}$, we have

$$
a_{n}^{k}=\frac{f_{n}^{\nu^{k+1}}-f_{n}^{v^{k}}}{\mu^{k}}=\frac{f_{n}-f_{n}}{\mu^{k}}=0 .
$$

Combining this and (4.15), we conclude that $a^{k}$ is a $(\varphi, \infty)_{M}$-atom.

Now we show that $\left\{\mu^{k}, a^{k}, v^{k}\right\}_{k \in \mathbb{Z}} \in \mathcal{A}_{M}(\varphi, \infty)$. To this end, for any $k \in \mathbb{Z}$, let $G_{k}:=B_{v^{k}} \backslash B_{v^{k+1}}$. Similarly to the proof of (4.3), we find that, for any given $r \in(0, \infty)$,

$$
\sum_{k \in \mathbb{Z}}\left[\frac{\mu^{k} \mathbf{1}_{B_{v^{k}}}}{\left\|\mathbf{1}_{B_{\nu^{k}}}\right\|_{L^{\varphi}(\Omega)}}\right]^{r}=\frac{6^{r}}{2^{r}-1} \sum_{k \in \mathbb{Z}}\left(2^{k} \mathbf{1}_{G_{k}}\right)^{r}
$$

Combining this, (4.13) and the fact that $\left\{G_{k}\right\}_{k \in \mathbb{Z}}$ are disjoint, we know that, for any given $r \in(0, \infty)$ and any $\lambda \in(0, \infty)$,

$$
\begin{aligned}
\int_{\Omega} \varphi\left(x, \frac{1}{\lambda}\left\{\sum_{k \in \mathbb{Z}}\left[\frac{\mu^{k} \mathbf{1}_{B_{\nu^{k}}}(x)}{\left\|\mathbf{1}_{B_{v^{k}}}\right\|_{L^{\varphi}}(\Omega)}\right]^{r}\right\}^{\frac{1}{r}}\right) d \mathbb{P}(x) & =\int_{\Omega} \varphi\left(x, \frac{1}{\lambda}\left(\frac{6^{r}}{2^{r}-1}\right)^{\frac{1}{r}} \sum_{k \in \mathbb{Z}} 2^{k} \mathbf{1}_{G_{k}}(x)\right) d \mathbb{P}(x) \\
& =\sum_{k \in \mathbb{Z}} \int_{G_{k}} \varphi\left(x,\left(\frac{6^{r}}{2^{r}-1}\right)^{\frac{1}{r}} \frac{2^{k}}{\lambda}\right) d \mathbb{P}(x) \\
& \leq \sum_{k \in \mathbb{Z}} \int_{B_{v^{k}}} \varphi\left(x,\left(\frac{6^{r}}{2^{r}-1}\right)^{\frac{1}{r}} \frac{2^{k}}{\lambda}\right) d \mathbb{P}(x) \\
& \leq R K \sum_{k \in \mathbb{Z}} \int_{\left\{x \in \Omega: M(f)(x)>2^{k}\right\}} \varphi\left(x,\left(\frac{6^{r}}{2^{r}-1}\right)^{\frac{1}{r}} \frac{2^{k}}{\lambda}\right) d \mathbb{P}(x)=: \text { I }
\end{aligned}
$$

Since $\varphi$ is of uniformly lower type $p \in(0, \infty)$, we know that, for any given $r \in(0, \infty)$ and any $\lambda \in(0, \infty)$,

$$
\mathrm{I} \lesssim \sum_{k \in \mathbb{Z}} \sum_{j=k}^{\infty} \int_{\left\{x \in \Omega: 2^{j}<M(f)(x) \leq 2^{j+1}\right\}} \varphi\left(x,\left(\frac{6^{r}}{2^{r}-1}\right)^{\frac{1}{r}} \frac{2^{k}}{\lambda}\right) d \mathbb{P}(x)
$$




$$
\begin{aligned}
& \lesssim \sum_{k \in \mathbb{Z}} \sum_{j=k}^{\infty} 2^{(k-j) p} \int_{\left\{x \in \Omega: 2^{j}<M(f)(x) \leq 2^{j+1}\right\}} \varphi\left(x,\left(\frac{6^{r}}{2^{r}-1}\right)^{\frac{1}{r}} \frac{2^{j}}{\lambda}\right) d \mathbb{P}(x) \\
& \lesssim \sum_{j \in \mathbb{Z}} \sum_{k \leq j} 2^{(k-j) p} \int_{\left\{x \in \Omega: 2^{j}<M(f)(x) \leq 2^{j+1}\right\}} \varphi\left(x,\left(\frac{6^{r}}{2^{r}-1}\right)^{\frac{1}{r}} \frac{M(f)(x)}{\lambda}\right) d \mathbb{P}(x) \\
& \lesssim \int_{\Omega} \varphi\left(x,\left(\frac{6^{r}}{2^{r}-1}\right)^{\frac{1}{r}} \frac{M(f)(x)}{\lambda}\right) d \mathbb{P}(x) .
\end{aligned}
$$

From this, we deduce that, for any $\lambda \in(0, \infty)$,

$$
\int_{\Omega} \varphi\left(x, \frac{1}{\lambda}\left\{\sum_{k \in \mathbb{Z}}\left[\frac{\mu^{k} \mathbf{1}_{B_{\gamma^{k}}}(x)}{\left\|\mathbf{1}_{B_{\gamma^{k}}}\right\|_{L^{\varphi}(\Omega)}}\right]^{r}\right\}^{\frac{1}{r}}\right) d \mathbb{P}(x) \lesssim \int_{\Omega} \varphi\left(x,\left(\frac{6^{r}}{2^{r}-1}\right)^{\frac{1}{r}} \frac{M(f)(x)}{\lambda}\right) d \mathbb{P}(x),
$$

which, together with the fact that $\varphi$ is of uniformly lower type $p$, implies that, for any given $r \in(0, \infty)$,

$$
\left\|\left\{\sum_{k \in \mathbb{Z}}\left[\frac{\mu^{k} \mathbf{1}_{B_{v^{k}}}(x)}{\left\|\mathbf{1}_{B_{v^{k}}}\right\|_{L^{\varphi}(\Omega)}}\right]^{r}\right\}^{\frac{1}{r}}\right\|_{L^{\varphi}(\Omega)} \lesssim\|f\|_{H_{\varphi}^{M}(\Omega)} .
$$

By this, we further know that, for any given $r \in(0, \infty), f \in H_{\mathrm{at}, r}^{\varphi, \infty, M}(\Omega)$ and $\|f\|_{H_{\mathrm{at}, r}^{\varphi, \infty, M}(\Omega)} \lesssim$ $\|f\|_{H_{\varphi}^{M}(\Omega)}$. The proof of Step 1) is now complete.

Step 2) Prove $H_{\mathrm{at}, r}^{\varphi, \infty}(\Omega) \subset H_{\varphi}^{M}(\Omega)$. To show this, for any given $r \in(0,1]$, Let $f \in H_{\mathrm{at}, r}^{\varphi, \infty}(\Omega)$. Then we can write $f=\sum_{k \in \mathbb{Z}} \mu^{k} a^{k}$ for some sequence of triples,

$$
\left\{\mu^{k}, a^{k}, v^{k}\right\}_{k \in \mathbb{Z}} \in \mathcal{A}_{M}(\varphi, \infty)
$$

By Definition 2.3(i), we know that, for any $k \in \mathbb{Z}$,

$$
\left\{x \in \Omega: M\left(a^{k}\right)(x) \neq 0\right\} \subset B_{v^{k}} .
$$

Thus, for almost every $x \in \Omega$, we have

$$
M\left(a^{k}\right)(x) \leq\left\|M\left(a^{k}\right)\right\|_{L^{\infty}(\Omega)} \mathbf{1}_{B_{v^{k}}}(x) \leq \mathbf{1}_{B_{v^{k}}}(x)\left\|\mathbf{1}_{B_{v^{k}}}\right\|_{L^{\varphi}(\Omega)}^{-1} .
$$

Combining this and the subadditivity of the operator $M$, we find that, for any given $r \in(0,1]$ and any $\lambda \in(0, \infty)$,

$$
\begin{aligned}
\int_{\Omega} \varphi\left(x, \frac{M(f)(x)}{\lambda}\right) d \mathbb{P}(x) & \leq \int_{\Omega} \varphi\left(x, \sum_{k \in \mathbb{Z}} \frac{\mu^{k} M\left(a^{k}\right)(x)}{\lambda}\right) d \mathbb{P}(x) \leq \int_{\Omega} \varphi\left(x, \sum_{k \in \mathbb{Z}} \frac{\mu^{k} \mathbf{1}_{B_{v^{k}}}(x)}{\lambda\left\|\mathbf{1}_{B_{v^{k}}}\right\|_{L^{\varphi}(\Omega)}}\right) d \mathbb{P}(x) \\
& \leq \int_{\Omega} \varphi\left(x, \frac{1}{\lambda}\left\{\sum_{k \in \mathbb{Z}}\left[\frac{\mu^{k} \mathbf{1}_{B_{v^{k}}}(x)}{\left\|\mathbf{1}_{B_{v^{k}}}\right\|_{L^{\varphi}}(\Omega)}\right]^{r}\right\}^{\frac{1}{r}}\right) d \mathbb{P}(x) .
\end{aligned}
$$

Therefore, we conclude that, for any given $r \in(0,1], f \in H_{\varphi}^{M}(\Omega)$ and $\|f\|_{H_{\varphi}^{M}(\Omega)} \leq\|f\|_{H_{\mathrm{at}, r}^{\varphi, \infty, M}(\Omega)}$, which completes the proof of Theorem 4.6. 
Remark 4.8. From Example 2.17, it follows that Theorems 4.1, 4.4 and 4.6 cover the atomic characterizations of the variable martingale Hardy spaces in [40, Theorem 3.11, Theorem 3.12 and Proposition 4.19]. Let $w$ be a special weight and $\Phi$ be an Orlicz function. For any $x \in \Omega$ and $t \in(0, \infty)$, if $\varphi(x, t):=w(x) \Phi(t)$, then Theorems 4.1, 4.4 and 4.6 give the atomic characterizations of the weighted martingale Orlicz Hardy spaces, which is also new.

\section{Martingale inequalities}

Let $X$ be a martingale space and $Y$ a measurable function space. An operator $T: X \rightarrow Y$ is said to be a $\sigma$-sublinear operator if, for any complex number $\alpha$,

$$
\left|T\left(\sum_{k=1}^{\infty} f_{k}\right)\right| \leq \sum_{k=1}^{\infty}\left|T\left(f_{k}\right)\right| \quad \text { and } \quad|T(\alpha f)|=|\alpha||T(f)| .
$$

Lemma 5.1. Let a be a measurable function. If there exists a stopping time $v$ such that, for any $n \in \mathbb{N}$ with $n \leq v, \mathbb{E}_{n}(a)=0$, then, for any $n \in \mathbb{N}$,

$$
\mathbb{E}_{n}\left(a \mathbf{1}_{A}\right)=\mathbb{E}_{n}(a) \mathbf{1}_{A}, \quad \forall A \in \mathcal{F}_{v} .
$$

Moreover, let $T$ be any one of operators $S$, $s$ and $M$. Then, for any $(\varphi, \infty)_{T}$-atom a,

$$
T\left(a \mathbf{1}_{A}\right)=T(a) \mathbf{1}_{A}, \quad \forall A \in \mathcal{F}_{\nu},
$$

where $v$ is the stopping time associated with a.

Proof. Since $A \in \mathcal{F}_{v}$, it follows that, for any $n \in \mathbb{N}, A \cap\{v<n\} \in \mathcal{F}_{n-1}$. By this, we find that, for any $n \in \mathbb{N}$,

$$
\mathbb{E}_{n}\left(a \mathbf{1}_{A}\right)=\mathbb{E}_{n}\left(a \mathbf{1}_{A \cap\{v<n\}}+a \mathbf{1}_{A \cap\{v \geq n\}}\right)=\mathbb{E}_{n}(a) \mathbf{1}_{A \cap\{v<n\}}+\mathbb{E}_{n}\left(a \mathbf{1}_{A \cap\{v \geq n\}}\right) .
$$

Now we claim that, for any $n \in \mathbb{N}, \mathbb{E}_{n}\left(a \mathbf{1}_{A \cap\{v \geq n\}}\right)=0$. Indeed, for any $m \in \mathbb{N}$ and $m \geq n$, we have $\mathbb{E}_{m}(a) \mathbf{1}_{\{v=m\}}=0$. Combining this and $A \cap\{v=m\} \in \mathcal{F}_{m}$, we know that, for any $B \in \mathcal{F}_{n}$,

$$
\int_{B} a \mathbf{1}_{A \cap\{v \geq n\}} d \mathbb{P}=\sum_{m=n}^{\infty} \int_{B \cap A \cap\{v=m\}} a d \mathbb{P}=\sum_{m=n}^{\infty} \int_{B \cap A \cap\{v=m\}} \mathbb{E}_{m} a d \mathbb{P}=0 .
$$

This proves the above claim. Notice that, for any $n \in \mathbb{N}, \mathbb{E}_{n}(a)=0$ on the set $\{x \in \Omega: v(x) \geq n\}$. Therefore, we conclude that, for any $n \in \mathbb{N}$,

$$
\mathbb{E}_{n}\left(a \mathbf{1}_{A}\right)=\mathbb{E}_{n}(a) \mathbf{1}_{A \cap\{v<n\}}=\mathbb{E}_{n}(a) \mathbf{1}_{A} .
$$

From this, it follows that, for any $(\varphi, \infty)_{T}$-atom $a$,

$$
T\left(a \mathbf{1}_{A}\right)=T(a) \mathbf{1}_{A}, \quad \forall A \in \mathcal{F}_{v},
$$

where $v$ is the stopping time associated with $a$ and $T$ any one of operators $S, s$ and $M$. This finishes the proof of Lemma 5.1. 
Let $w$ be a special weight and $p \in(0, \infty)$. For any $x \in \Omega$ and $t \in(0, \infty)$, let $\varphi(x, t):=w(x) t^{p}$. Let us denote the corresponding Musielak-Orlicz Hardy spaces by $H_{p}^{M}(\Omega, w d \mathbb{P}), H_{p}^{S}(\Omega, w d \mathbb{P})$, $H_{p}^{s}(\Omega, w d \mathbb{P}), Q_{p}(\Omega, w d \mathbb{P})$ and $P_{p}(\Omega, w d \mathbb{P})$, respectively.

Theorem 5.2. Let $w \in A_{\infty}(\Omega) \cap \mathbb{S}^{-}$be a special weight and $p \in(0, \infty)$. If, for any $q \in(2, \infty)$, $T: H_{q}^{S}(\Omega) \rightarrow L^{q}(\Omega)\left(\right.$ resp., $\left.T: H_{q}^{M}(\Omega) \rightarrow L^{q}(\Omega)\right)$ is a bounded $\sigma$-sublinear operator and, for any $(\varphi, \infty)_{S}$-atoms (resp., $(\varphi, \infty)_{M}$-atoms) a,

$$
T(a) \mathbf{1}_{A}=T\left(a \mathbf{1}_{A}\right), \quad \forall A \in \mathcal{F}_{v},
$$

where $v$ is the associated stopping time with a, then there exists a positive constant $C$ such that, for any $f \in Q_{p}(\Omega, w d \mathbb{P})\left(\right.$ resp., $\left.f \in P_{p}(\Omega, w d \mathbb{P})\right)$,

$$
\|T(f)\|_{L^{p}(\Omega, w d \mathbb{P})} \leq C\|f\|_{Q_{p}(\Omega, w d \mathbb{P})} \quad\left(\text { resp. },\|T(f)\|_{L^{p}(\Omega, w d \mathbb{P})} \leq C\|f\|_{P_{p}(\Omega, w d \mathbb{P})}\right) .
$$

Proof. For any $p \in(0, \infty), x \in \Omega$ and $t \in(0, \infty)$, let $\varphi(x, t):=w(x) t^{p}$. For any given $f \in Q_{\varphi}(\Omega)$, by Theorem 4.4 , we know that there exists a sequence of triples, $\left\{\mu^{k}, a^{k}, v^{k}\right\}_{k \in \mathbb{Z}} \in \mathcal{A}_{S}(\varphi, \infty)$, such that $f=\sum_{k \in \mathbb{Z}} \mu^{k} a^{k}$ and

$$
\left\|\left[\sum_{k \in \mathbb{Z}} 2^{(k+1) r} \mathbf{1}_{B_{v^{k}}}\right]^{\frac{1}{r}}\right\|_{L^{\varphi}(\Omega)}=\left\|\left\{\sum_{k \in \mathbb{Z}}\left[\frac{\mu^{k} \mathbf{1}_{B_{v^{k}}}}{\left\|\mathbf{1}_{B_{v^{k}}}\right\|_{L^{\varphi}(\Omega)}}\right]^{r}\right\}^{\frac{1}{r}}\right\|_{L^{\varphi}(\Omega)} \lesssim\|f\|_{Q_{\varphi}(\Omega)},
$$

where $r \in(0,1], a^{k}$ is a $(\varphi, \infty)_{S}$-atom with the associated stopping time $v^{k}$ and $\mu^{k}=2^{k+1}\left\|\mathbf{1}_{B_{\gamma^{k}}}\right\|_{L^{\varphi}(\Omega)}$ for any $k \in \mathbb{Z}$. By the $\sigma$-sublinearity of the operator $T$, we have

$$
|T(f)| \leq \sum_{k \in \mathbb{Z}} \mu^{k}\left|T\left(a^{k}\right)\right| .
$$

From this and Lemma 2.12, it follows that, for any $r \in(0,1]$,

$$
\|T(f)\|_{L^{\varphi}(\Omega)} \leq\left\|\left\{\sum_{k \in \mathbb{Z}}\left[\mu^{k}\left|T\left(a^{k}\right)\right|\right]^{r}\right\}^{\frac{1}{r}}\right\|_{L^{\varphi}(\Omega)}=\left\|\sum_{k \in \mathbb{Z}}\left[\mu^{k}\left|T\left(a^{k}\right)\right|\right]^{r}\right\|_{L^{\varphi_{1 / r}(\Omega)}}^{\frac{1}{r}},
$$

where $\varphi_{1 / r}$ is as in Definition 2.11. By this, Lemma 2.18, (5.1) and the fact that $a^{k}=a^{k} \mathbf{1}_{B_{v^{k}}}$ for any $k \in \mathbb{Z}$, we may choose a function $g \in L^{\varphi_{1 / r}^{*}}(\Omega)$ with norm less than or equal to 1 such that, for any $r \in(0, \min \{1, p\}]$,

$$
\begin{aligned}
\|T(f)\|_{L^{\varphi}(\Omega)}^{r} & \lesssim \int_{\Omega} \sum_{k \in \mathbb{Z}}\left[\mu^{k}\left|T\left(a^{k}\right)\right|\right]^{r} g d \mathbb{P} \\
& \lesssim \sum_{k \in \mathbb{Z}} \int_{\Omega} 2^{(k+1) r}\left\|\mathbf{1}_{B_{v^{k}}}\right\|_{L^{\varphi}(\Omega)}^{r} \mathbf{1}_{B_{v^{k}}} \mathbb{E}_{v^{k}}\left(\left|T\left(a^{k}\right)\right|^{r}|g|\right) d \mathbb{P} .
\end{aligned}
$$

From the Hölder inequality, we deduce that, for any $r \in(0, \min \{1, p\}]$ and $q \in(\max \{2, p\}, \infty)$,

$$
\|T(f)\|_{L^{\varphi}(\Omega)}^{r} \lesssim \sum_{k \in \mathbb{Z}} \int_{\Omega} 2^{(k+1) r} \mathbf{1}_{B_{v^{k}}}\left\|\mathbf{1}_{B_{v^{k}}}\right\|_{L^{\varphi}(\Omega)}^{r}\left[\mathbb{E}_{v^{k}}\left(\left|T\left(a^{k}\right)\right|^{q}\right)\right]^{r / q}\left[\mathbb{E}_{v^{k}}\left(|g|^{(q / r)^{\prime}}\right)\right]^{1 /(q / r)^{\prime}} d \mathbb{P}
$$


For any $k \in \mathbb{Z}$ and $A \in \mathcal{F}_{v^{k}}$, by (5.1), Lemma 5.1 and the boundedness of $T$ from $H_{q}^{S}(\Omega)$ to $L^{q}(\Omega)$, we find that

$$
\int_{A}\left|T\left(a^{k}\right)\right|^{q} d \mathbb{P}=\int_{\Omega}\left|T\left(a^{k} \mathbf{1}_{A}\right)\right|^{q} d \mathbb{P} \lesssim \int_{\Omega}\left[S\left(a^{k} \mathbf{1}_{A}\right)\right]^{q} d \mathbb{P} \lesssim \int_{A}\left[S\left(a^{k}\right)\right]^{q} d \mathbb{P} .
$$

By this and the fact that $a^{k}$ is a $(\varphi, \infty)_{S}$-atom, we know that, for any $A \in \mathcal{F}_{v^{k}}$,

$$
\int_{A}\left|T\left(a^{k}\right)\right|^{q} d \mathbb{P} \lesssim\left\|\mathbf{1}_{B_{\nu^{k}}}\right\|_{L^{\varphi}(\Omega)}^{-q} \mathbb{P}(A),
$$

which implies that, for almost every $x \in \Omega$,

$$
\mathbb{E}_{v^{k}}\left(\left|T\left(a^{k}\right)\right|^{q}\right)(x) \lesssim\left\|\mathbf{1}_{B_{v^{k}}}\right\|_{L^{\varphi}(\Omega)}^{-q} .
$$

From this, (5.3) and Lemma 2.18, it follows that, for any $r \in(0, \min \{1, p\}]$ and $q \in(\max \{2, p\}, \infty)$,

$$
\begin{aligned}
\|T(f)\|_{L^{\varphi}(\Omega)}^{r} & \lesssim \sum_{k \in \mathbb{Z}} \int_{\Omega} 2^{(k+1) r} \mathbf{1}_{B_{v^{k}}}\left[\mathbb{E}_{v^{k}}\left(|g|^{(q / r)^{\prime}}\right)\right]^{1 /(q / r)^{\prime}} d \mathbb{P} \\
& \lesssim \int_{\Omega} \sum_{k \in \mathbb{Z}} 2^{(k+1) r} \mathbf{1}_{B_{\nu^{k}}}\left[M\left(|g|^{(q / r)^{\prime}}\right)\right]^{1 /(q / r)^{\prime}} d \mathbb{P} \\
& \lesssim\left\|\sum_{k \in \mathbb{Z}} 2^{(k+1) r} \mathbf{1}_{B_{v^{k}}}\right\|_{L_{\varphi_{1 / r}}(\Omega)}\left\|\left[M\left(|g|^{(q / r)^{\prime}}\right)\right]^{1 /(q / r)^{\prime}}\right\|_{L_{\varphi_{1 / r}^{*}}(\Omega)} .
\end{aligned}
$$

If $p / r>1$, Example 2.16(i) tells us that $\varphi_{1 / r}^{*}(x, t)=w(x)^{1-(p / r)^{\prime}} t^{(p / r)^{\prime}} p^{-\frac{1}{p-1}}$ for any $x \in \Omega$ and $t \in(0, \infty)$, and hence we can write the second norm in the last expression of (5.4) as

$$
\left\|\left[M\left(|g|^{(q / r)^{\prime}}\right)\right]^{1 /(q / r)^{\prime}}\right\|_{L_{\varphi_{1 / r}^{*}}(\Omega)}^{(p / r)^{\prime}}=p^{-\frac{1}{p-1}} \int_{\Omega}\left[M\left(|g|^{(q / r)^{\prime}}\right)\right]^{(p / r)^{\prime} /(q / r)^{\prime}} w^{1-(p / r)^{\prime}} d \mathbb{P} .
$$

Since $w \in A_{\infty}(\Omega)$, it follows that there exists $r \in(0, \min \{1, p\}]$ and $r \neq p$ such that $w \in A_{p / r}(\Omega)$. From this, it follows that $w^{1-(p / r)^{\prime}} \in A_{(p / r)^{\prime}}(\Omega)$. By Lemma 2.7 and $w \in \mathbb{S}^{-}$, we know that there exists $\varepsilon \in(0, \infty)$ such that $w^{1-(p / r)^{\prime}} \in A_{(p / r)^{\prime}-\varepsilon}(\Omega)$. We can choose $q$ large enough such that

$$
(p / r)^{\prime} /(q / r)^{\prime}>(p / r)^{\prime}-\varepsilon
$$

By this, (5.5) and the Doob maximal inequality (Theorem 3.2), we have

$$
\left\|\left[M\left(|g|^{(q / r)^{\prime}}\right)\right]^{1 /(q / r)^{\prime}}\right\|_{L_{\varphi_{1 / r}^{*}(\Omega)}^{(p / r)^{\prime}}}^{\left(\int_{\Omega}\right.}|g|^{(p / r)^{\prime}} w^{1-(p / r)^{\prime}} d \mathbb{P} \lesssim 1,
$$

because $\|g\|_{L^{\varphi_{1 / r}^{*}(\Omega)}} \leq 1$. From this, (5.2) and (5.4), we deduce that

$$
\|T(f)\|_{L^{\varphi}(\Omega)} \lesssim\left\|\sum_{k \in \mathbb{Z}} 2^{(k+1) r} \mathbf{1}_{B_{\nu^{k}}}\right\|_{L_{\varphi_{1 / r}}(\Omega)}^{1 / r} \lesssim\left\|\left[\sum_{k \in \mathbb{Z}} 2^{(k+1) r} \mathbf{1}_{B_{v^{k}}}\right]^{\frac{1}{r}}\right\|_{L^{\varphi}(\Omega)} \lesssim\|f\|_{Q_{\varphi}(\Omega)},
$$

which completes the proof for $Q_{\varphi}(\Omega)$. The proof for $P_{\varphi}(\Omega)$ is similar. This finishes the proof of Theorem 5.2. 
Similarly to the proof of Theorem 5.2, we can prove the following theorem.

Theorem 5.3. Let $w \in A_{\infty}(\Omega) \cap \mathbb{S}^{-}$be a special weight and $p \in(0,2)$. If $T: H_{2}^{s}(\Omega) \rightarrow L^{2}(\Omega)$ is a bounded $\sigma$-sublinear operator and, for any $(\varphi, \infty)_{s}$-atom $a$,

$$
T(a) \mathbf{1}_{A}=T\left(a \mathbf{1}_{A}\right), \quad \forall A \in \mathcal{F}_{v},
$$

where $v$ is the associated stopping time with a, then there exists a positive constant $C$ such that, for any $f \in H_{p}^{s}(\Omega, w d \mathbb{P})$,

$$
\|T(f)\|_{L^{p}(\Omega, w d \mathbb{P})} \leq C\|f\|_{H_{p}^{s}(\Omega, w d \mathbb{P})} .
$$

Proof. For any $\varepsilon \in(0,1)$, we choose $r$ small enough such that $(p / r)^{\prime} /(2 / r)^{\prime}>(p / r)^{\prime}-\varepsilon$, this is due to

$$
\lim _{r \rightarrow 0} \frac{(p / r)^{\prime}}{(p / r)^{\prime}-\varepsilon}=\frac{1}{1-\varepsilon} \quad \text { and } \quad \lim _{r \rightarrow 0}(2 / r)^{\prime}=1 .
$$

Combing this and the proof of Theorem 5.2 with $q$ replaced by 2 , we obtain the desired conclusion. This finishes the proof of Theorem 5.3.

Corollary 5.4. Let $w \in A_{\infty}(\Omega) \cap \mathbb{S}^{-}$be a special weight.

(i) If $p \in(0, \infty)$, then there exists a positive constant $C$ such that, for any $f \in \mathcal{M}$,

$$
\begin{aligned}
\|f\|_{H_{p}^{M}(\Omega, w d \mathbb{P})} \leq C\|f\|_{P_{p}(\Omega, w d \mathbb{P}),} & \|f\|_{H_{p}^{S}(\Omega, w d \mathbb{P})} \leq C\|f\|_{Q_{p}(\Omega, w d \mathbb{P}),} \\
\|f\|_{H_{p}^{S}(\Omega, w d \mathbb{P})} \leq C\|f\|_{P_{p}(\Omega, w d \mathbb{P}),} & \|f\|_{H_{p}^{M}(\Omega, w d \mathbb{P})} \leq C\|f\|_{Q_{p}(\Omega, w d \mathbb{P})}
\end{aligned}
$$

and

$$
\|f\|_{H_{p}^{s}(\Omega, w d \mathbb{P})} \leq C\|f\|_{P_{p}(\Omega, w d \mathbb{P})}, \quad\|f\|_{H_{p}^{s}(\Omega, w d \mathbb{P})} \leq C\|f\|_{Q_{p}(\Omega, w d \mathbb{P})} .
$$

(ii) If $p \in(0,2)$, then there exists a positive constant $C$ such that, for any $f \in \mathcal{M}$,

$$
\|f\|_{H_{p}^{M}(\Omega, w d \mathbb{P})} \leq C\|f\|_{H_{p}^{s}(\Omega, w d \mathbb{P})}, \quad\|f\|_{H_{p}^{S}(\Omega, w d \mathbb{P})} \leq C\|f\|_{H_{p}^{s}(\Omega, w d \mathbb{P})} .
$$

Proof. The two inequalities in (5.7) follow easily from Definition 2.1. For the two inequalities in (5.8), consider the operator $T=M$ or $S$ in Theorem 5.2. Then the both inequalities in (5.8) follow from the Burkholder-Gundy inequality

$$
\|S(f)\|_{L^{q}(\Omega)} \sim\|M(f)\|_{L^{q}(\Omega)}, \quad \forall q \in(1, \infty)
$$

(see, for example, [77, Theorem 2.12]), Lemma 5.1 and Theorem 5.2.

The both inequalities in (5.9) can be deduced from choosing $T=s$ and applying the inequality

$$
\|s(f)\|_{L^{q}(\Omega)} \lesssim\|M(f)\|_{L^{q}(\Omega)} \sim\|S(f)\|_{L^{q}(\Omega)}, \quad \forall q \in(2, \infty)
$$

(see, for example, [77, Theorem 2.11(ii)]), Lemma 5.1 and Theorem 5.2.

In order to prove the both inequalities in (5.10), consider the operator $T=M$ or $S$ in Theorem 5.3. Then the desired inequalities in (5.10) follow immediately from

$$
\|S(f)\|_{L^{2}(\Omega)} \lesssim\|s(f)\|_{L^{2}(\Omega)} \quad \text { and } \quad\|M(f)\|_{L^{2}(\Omega)} \lesssim\|s(f)\|_{L^{2}(\Omega)}
$$

(see [77, Theorem 2.11(i)] or [11, Theorem 5.3(ii)]), Lemma 5.1 and Theorem 5.3. This finishes the proof of Corollary 5.4. 
Remark 5.5. Inequalities (5.10) with a special class of weights were first studied by Kazamaki [42, Theorem 1]. Since the weight in (5.10) is of wide generality, (5.10) generalizes [42, Theorem $1]$ in the case when $p \in(0,2)$.

Theorem 5.6. Let $\varphi \in A_{\infty}(\Omega) \cap \mathbb{S}^{-}$be a Musielak-Orlicz function with uniformly lower type $p_{\varphi}^{-}$ and uniformly upper type $p_{\varphi}^{+}$satisfying $0<p_{\varphi}^{-} \leq p_{\varphi}^{+}<\infty$. Suppose that the $\sigma$-sublinear operator $T$ satisfies (5.1) and, for some $q \in\left(\max \left\{1, p_{\varphi}^{+}\right\}, \infty\right)$ and any $t \in(0, \infty)$,

$$
\|T(f)\|_{L^{q}(\Omega, \varphi(\cdot, t) d \mathbb{P})} \leq C\|f\|_{H_{q}^{s}(\Omega, \varphi(\cdot, t) d \mathbb{P})}, \quad \forall f \in H_{q}^{s}(\Omega, \varphi(\cdot, t) d \mathbb{P}),
$$

where $C$ is a positive constant independent of $f$ and $t \in(0, \infty)$. Then there exists a positive constant $C$ such that, for any $f \in H_{\varphi}^{s}(\Omega)$,

$$
\|T(f)\|_{L^{\varphi}(\Omega)} \leq C\|f\|_{H_{\varphi}^{s}(\Omega)} .
$$

The same holds true if one replaces the spaces $H_{q}^{s}(\Omega, \varphi(\cdot, t) d \mathbb{P})$ and $H_{\varphi}^{s}(\Omega)$ by $Q_{q}(\Omega, \varphi(\cdot, t) d \mathbb{P})$ and $Q_{\varphi}(\Omega)$ or by $P_{q}(\Omega, \varphi(\cdot, t) d \mathbb{P})$ and $P_{\varphi}(\Omega)$, respectively.

Proof. Let $f \in H_{\varphi}^{s}(\Omega)$. Then, by Theorem 4.1, we know that there exists a sequence of triples, $\left\{\mu^{k}, a^{k}, v^{k}\right\}_{k \in \mathbb{Z}} \in \mathcal{A}_{s}(\varphi, \infty)$, such that $f=\sum_{k \in \mathbb{Z}} \mu^{k} a^{k}$ and, for any $\lambda \in(0, \infty)$,

$$
\int_{\Omega} \varphi\left(x, \frac{2}{\lambda} \sum_{k \in \mathbb{Z}} 2^{k+1} \mathbf{1}_{G_{k}}(x)\right) d \mathbb{P}(x) \leq \int_{\Omega} \varphi\left(x, 4 \frac{s(f)(x)}{\lambda}\right) d \mathbb{P}(x),
$$

where $v^{k}:=\inf \left\{n \in \mathbb{N}: s_{n+1}(f)>2^{k}\right\}, \mu^{k}:=2^{k+1}\left\|\mathbf{1}_{B_{\gamma^{k}}}\right\|_{L^{\varphi}(\Omega)}$ and $G_{k}:=B_{\gamma^{k}} \backslash B_{\gamma^{k+1}}$ for any $k \in \mathbb{Z}$ (see (4.4)). Thus, for any $k \in \mathbb{Z}$, we have $G_{k}=\left\{x \in \Omega: 2^{k}<s(f)(x) \leq 2^{k+1}\right\}$. Since $\left\{G_{k}\right\}_{k \in \mathbb{Z}}$ are disjoint, we obtain, for any $k \in \mathbb{Z}, \mathbf{1}_{B_{v^{k}}}=\sum_{j=k}^{\infty} \mathbf{1}_{G_{j}}$. From this, it follows that, for any $\lambda \in(0, \infty)$,

$$
\begin{aligned}
\int_{\Omega} \varphi\left(x, \frac{|T(f)(x)|}{\lambda}\right) d \mathbb{P}(x) & \leq \int_{\Omega} \varphi\left(x, \frac{1}{\lambda} \sum_{k \in \mathbb{Z}} \mu^{k}\left|T\left(a^{k}\right)(x)\right| \mathbf{1}_{B_{v^{k}}}(x)\right) d \mathbb{P}(x) \\
& =\int_{\Omega} \varphi\left(x, \frac{1}{\lambda} \sum_{k \in \mathbb{Z}} \sum_{j=k}^{\infty} \mu^{k}\left|T\left(a^{k}\right)(x)\right| \mathbf{1}_{G_{j}}(x)\right) d \mathbb{P}(x) \\
& =\sum_{j \in \mathbb{Z}} \int_{G_{j}} \varphi\left(x, \frac{1}{\lambda} \sum_{k=-\infty}^{j} 2^{k+1}\left\|\mathbf{1}_{B_{v^{k}}}\right\|_{L^{\varphi}(\Omega)}\left|T\left(a^{k}\right)(x)\right|\right) d \mathbb{P}(x) \\
& =: \text { I. }
\end{aligned}
$$

Let $q \in\left(\max \left\{p_{\varphi}^{+}, 1\right\}, \infty\right)$ and $\ell \in(0,1)$ be such that $q(1-\ell)=p_{\varphi}^{+}$. Since $\varphi$ is of uniformly upper type $p_{\varphi}^{+}$, we know that $\varphi$ is of uniformly upper type $q$. From this, the Hölder inequality and the fact that $\left\{G_{k}\right\}_{k \in \mathbb{Z}}$ are disjoint, we deduce that, for any $\lambda \in(0, \infty)$,

$$
\mathrm{I} \lesssim \sum_{j \in \mathbb{Z}} \int_{G_{j}} \frac{1}{2^{(j+1) q}}\left[\sum_{k=-\infty}^{j} 2^{k+1}\left\|\mathbf{1}_{B_{v^{k}}}\right\|_{L^{\varphi}(\Omega)} \mid T\left(a^{k}\right)(x)\right]^{q} \varphi\left(x, \frac{2^{j+1}}{\lambda}\right) d \mathbb{P}(x)
$$




$$
\begin{aligned}
& +\sum_{j \in \mathbb{Z}} \int_{G_{j}} \varphi\left(x, \frac{2^{j+1}}{\lambda}\right) d \mathbb{P}(x) \\
& \lesssim \sum_{j \in \mathbb{Z}} \frac{1}{2^{(j+1) q}} \int_{G_{j}}\left(\sum_{k=-\infty}^{j} 2^{k \ell q^{\prime}}\right)^{\frac{q}{q}} \sum_{k=-\infty}^{j} 2^{-k \ell q} 2^{(k+1) q}\left\|\mathbf{1}_{B_{v^{k}}}\right\|_{L^{\varphi}(\Omega)}^{q}\left|T\left(a^{k}\right)(x)\right|^{q} \varphi\left(x, \frac{2^{j+1}}{\lambda}\right) d \mathbb{P}(x) \\
& +\int_{\Omega} \varphi\left(x, \frac{1}{\lambda} \sum_{j \in \mathbb{Z}} 2^{j+1} \mathbf{1}_{G_{j}}(x)\right) d \mathbb{P}(x) \\
& \lesssim \sum_{j \in \mathbb{Z}} \frac{1}{2^{j p_{\varphi}^{+}}} \sum_{k=-\infty}^{j} 2^{k p_{\varphi}^{+}}\left\|\mathbf{1}_{B_{v^{k}}}\right\|_{L^{\varphi}(\Omega)}^{q} \int_{G_{j}}\left|T\left(a^{k}\right)(x)\right|^{q} \varphi\left(x, \frac{2^{j+1}}{\lambda}\right) d \mathbb{P}(x) \\
& +\int_{\Omega} \varphi\left(x, \frac{1}{\lambda} \sum_{j \in \mathbb{Z}} 2^{j+1} \mathbf{1}_{G_{j}}(x)\right) d \mathbb{P}(x)=: \mathrm{I}_{1}+\mathrm{I}_{2} .
\end{aligned}
$$

Since $\varphi$ is of uniformly upper type $p_{\varphi}^{+}$, from (5.11), the fact that $\left\{G_{j}\right\}_{j \in \mathbb{Z}}$ are disjoint, $a^{k}$ is a $(\varphi, \infty)_{s}$-atom and $\bigcup_{j=k}^{\infty} G_{j}=B_{v^{k}}$ for any $k \in \mathbb{Z}$, we deduce that, for any $\lambda \in(0, \infty)$,

$$
\begin{aligned}
& \mathrm{I}_{1}=\sum_{k \in \mathbb{Z}} 2^{k p_{\varphi}^{+}}\left\|\mathbf{1}_{B_{v^{k}}}\right\|_{L^{\varphi}(\Omega)}^{q} \sum_{j=k}^{\infty} \frac{1}{2^{j p_{\varphi}^{+}}} \int_{G_{j}}\left|T\left(a^{k}\right)(x)\right|^{q} \varphi\left(x, \frac{2^{j+1}}{\lambda}\right) d \mathbb{P}(x) \\
& \lesssim \sum_{k \in \mathbb{Z}} 2^{k p_{\varphi}^{+}}\left\|\mathbf{1}_{B_{v^{k}}}\right\|_{L^{\varphi}(\Omega)}^{q} \sum_{j=k}^{\infty} \frac{1}{2^{j p_{\varphi}^{+}}} \int_{G_{j}}\left|T\left(a^{k}\right)(x)\right|^{q} 2^{(j+1-k) p_{\varphi}^{+}} \varphi\left(x, \frac{2^{k}}{\lambda}\right) d \mathbb{P}(x) \\
& \sim \sum_{k \in \mathbb{Z}}\left\|\mathbf{1}_{B_{v^{k}}}\right\|_{L^{\varphi}(\Omega)}^{q} \int_{B_{v^{k}}}\left|T\left(a^{k}\right)(x)\right|^{q} \varphi\left(x, \frac{2^{k}}{\lambda}\right) d \mathbb{P}(x) \\
& \lesssim \sum_{k \in \mathbb{Z}}\left\|\mathbf{1}_{B_{v^{k}}}\right\|_{L^{\varphi}(\Omega)}^{q} \int_{B_{\nu^{k}}}\left[s\left(a^{k}\right)(x)\right]^{q} \varphi\left(x, \frac{2^{k}}{\lambda}\right) d \mathbb{P}(x) \\
& \lesssim \sum_{k \in \mathbb{Z}}\left\|\mathbf{1}_{B_{\nu^{k}}}\right\|_{L^{\varphi}(\Omega)}^{q}\left\|s\left(a^{k}\right)\right\|_{L^{\infty}(\Omega)}^{q} \int_{B_{\nu^{k}}} \varphi\left(x, \frac{2^{k}}{\lambda}\right) d \mathbb{P}(x) \lesssim \sum_{k \in \mathbb{Z}} \int_{B_{\gamma^{k}}} \varphi\left(x, \frac{2^{k}}{\lambda}\right) d \mathbb{P}(x) .
\end{aligned}
$$

Combining this and the fact that $\varphi$ is of uniformly lower type $p_{\varphi}^{-}$and, for any $j \in \mathbb{Z}, s(f)>2^{j}$ on $G_{j}$, we know that, for any $\lambda \in(0, \infty)$,

$$
\begin{aligned}
\mathrm{I}_{1} & \lesssim \sum_{k \in \mathbb{Z}} \sum_{j=k}^{\infty} \int_{G_{j}} \varphi\left(x, \frac{2^{k}}{\lambda}\right) d \mathbb{P}(x) \lesssim \sum_{k \in \mathbb{Z}} \sum_{j=k}^{\infty} 2^{(k-j) p_{\varphi}^{-}} \int_{G_{j}} \varphi\left(x, \frac{2^{j}}{\lambda}\right) d \mathbb{P}(x) \\
& \lesssim \sum_{j \in \mathbb{Z}} \sum_{k=-\infty}^{j} 2^{(k-j) p_{\varphi}^{-}} \int_{G_{j}} \varphi\left(x, \frac{s(f)(x)}{\lambda}\right) d \mathbb{P}(x) \lesssim \int_{\Omega} \varphi\left(x, \frac{s(f)(x)}{\lambda}\right) d \mathbb{P}(x) .
\end{aligned}
$$

From this, it follows that, for any $\lambda \in(0, \infty)$,

$$
\mathrm{I} \lesssim \int_{\Omega} \varphi\left(x, \frac{s(f)(x)}{\lambda}\right) d \mathbb{P}(x)+\mathrm{I}_{2}
$$


Combining this and (5.14), we deduce that, for any $\lambda \in(0, \infty)$,

$$
\int_{\Omega} \varphi\left(x, \frac{|T(f)(x)|}{\lambda}\right) d \mathbb{P}(x) \lesssim \int_{\Omega} \varphi\left(x, \frac{s(f)(x)}{\lambda}\right) d \mathbb{P}(x)+\mathrm{I}_{2},
$$

which, together with (5.13), implies that, for any $\lambda \in(0, \infty)$,

$$
\int_{\Omega} \varphi\left(x, \frac{|T(f)(x)|}{\lambda}\right) d \mathbb{P}(x) \lesssim \int_{\Omega} \varphi\left(x, \frac{s(f)(x)}{\lambda}\right) d \mathbb{P}(x) .
$$

Thus, we complete the proof of (5.12).

Assume now that $f \in Q_{\varphi}(\Omega)$. Then there exists an optimal control sequence $\left(\lambda_{n}^{(1)}(f)\right)_{n \in \mathbb{Z}_{+}}$such that $S_{n}(f) \leq \lambda_{n-1}^{(1)}(f)$ with $\lambda_{\infty}^{(1)}(f) \in L^{\varphi}(\Omega)$. If $a$ is a $(\varphi, \infty)_{S}$-atom, then $\lambda_{\infty}^{(1)}(a) \leq\|S(a)\|_{L^{\infty}(\Omega)}$. In the proof of (5.12), instead of (5.15), using (5.11), we conclude that, for any $\lambda \in(0, \infty)$,

$$
\begin{aligned}
\mathrm{I}_{1} & \lesssim \sum_{k \in \mathbb{Z}}\left\|\mathbf{1}_{B_{\gamma^{k}}}\right\|_{L^{\varphi}(\Omega)}^{q} \int_{B_{\gamma^{k}}}\left|T\left(a^{k}\right)(x)\right|^{q} \varphi\left(x, \frac{2^{k}}{\lambda}\right) d \mathbb{P}(x) \\
& \lesssim \sum_{k \in \mathbb{Z}}\left\|\mathbf{1}_{B_{\nu^{k}}}\right\|_{L^{\varphi}(\Omega)}^{q} \int_{B_{\gamma^{k}}}\left[\lambda_{\infty}^{(1)}\left(a^{k}\right)(x)\right]^{q} \varphi\left(x, \frac{2^{k}}{\lambda}\right) d \mathbb{P}(x) \\
& \lesssim \sum_{k \in \mathbb{Z}}\left\|\mathbf{1}_{B_{\nu^{k}}}\right\|_{L^{\varphi}(\Omega)}^{q}\left\|S\left(a^{k}\right)\right\|_{L^{\infty}(\Omega)}^{q} \int_{B_{\gamma^{k}}} \varphi\left(x, \frac{2^{k}}{\lambda}\right) d \mathbb{P}(x) \lesssim \sum_{k \in \mathbb{Z}} \int_{B_{\nu^{k}}} \varphi\left(x, \frac{2^{k}}{\lambda}\right) d \mathbb{P}(x) .
\end{aligned}
$$

Thus, the proof of (5.12) with $H_{\varphi}^{s}(\Omega)$ replaced by $Q_{\varphi}(\Omega)$ can be finished as above. The proof of (5.12) with $H_{\varphi}^{s}(\Omega)$ replaced by $P_{\varphi}(\Omega)$ is similar. This finishes the proof of Theorem 5.6.

Theorem 5.7. Let $\varphi \in A_{\infty}(\Omega)$ be a Musielak-Orlicz function with uniformly lower type $p_{\varphi}^{-}$and uniformly upper type $p_{\varphi}^{+}$satisfying $0<p_{\varphi}^{-} \leq p_{\varphi}^{+}<\infty$.

(i) If $\varphi \in \mathbb{S}^{-}$and $p_{\varphi}^{+} \in(0,2)$, then there exists a positive constant $C$ such that, for any $f \in$ $H_{\varphi}^{S}(\Omega)$

$$
\|f\|_{H_{\varphi}^{M}(\Omega)} \leq C\|f\|_{H_{\varphi}^{s}(\Omega)} .
$$

(ii) If $\varphi \in \mathbb{S}^{-}$and $p_{\varphi}^{+} \in(0,2)$, then there exists a positive constant $C$ such that, for any $f \in$ $H_{\varphi}^{s}(\Omega)$

$$
\|f\|_{H_{\varphi}^{S}(\Omega)} \leq C\|f\|_{H_{\varphi}^{s}(\Omega)} .
$$

(iii) If $\varphi \in \mathbb{S}^{-}$, then there exists a positive constant $C$ such that, for any $f \in Q_{\varphi}(\Omega)$,

$$
\begin{array}{cc}
\|f\|_{H_{\varphi}^{M}(\Omega)} \leq\|f\|_{P_{\varphi}(\Omega)}, & \|f\|_{H_{\varphi}^{S}(\Omega)} \leq\|f\|_{Q_{\varphi}(\Omega),}, \\
\|f\|_{H_{\varphi}^{S}(\Omega)} \leq C\|f\|_{P_{\varphi}(\Omega)}, & \|f\|_{H_{\varphi}^{M}(\Omega)} \leq C\|f\|_{Q_{\varphi}(\Omega),} \\
\|f\|_{H_{\varphi}^{s}(\Omega)} \leq C\|f\|_{P_{\varphi}(\Omega)}, & \|f\|_{H_{\varphi}^{s}(\Omega)} \leq C\|f\|_{Q_{\varphi}(\Omega)},
\end{array}
$$

and

$$
\frac{1}{C}\|f\|_{P_{\varphi}(\Omega)} \leq\|f\|_{Q_{\varphi}(\Omega)} \leq C\|f\|_{P_{\varphi}(\Omega)} .
$$


Moreover, if $\left\{\mathcal{F}_{n}\right\}_{n \in \mathbb{Z}_{+}}$is regular, then

$$
H_{\varphi}^{M}(\Omega)=P_{\varphi}(\Omega)=H_{\varphi}^{s}(\Omega)=H_{\varphi}^{S}(\Omega)=Q_{\varphi}(\Omega)
$$

with equivalent quasi-norms.

Proof. By Lemma 5.1, we know that the operators $M, S$ and $s$ are all satisfy (5.1). Then (5.16) and (5.17) follow from (5.10) and Theorem 5.6 with $T=M$ or $T=S$. Inequalities (5.18) come easily from the definition of $P_{\varphi}(\Omega)$ and $Q_{\varphi}(\Omega)$. Inequalities (5.19) and (5.20) follow from Corollary 5.4 and Theorem 5.6 by choosing $T=M, S$ or $s$.

To prove (5.21), we use (5.19). If $f=\left(f_{n}\right)_{n \in \mathbb{Z}_{+}} \in Q_{\varphi}(\Omega)$, then there exists an optimal control $\left(\lambda_{n}^{(1)}(f)\right)_{n \in \mathbb{Z}_{+}}$such that $S_{n}(f) \leq \lambda_{n-1}^{(1)}(f)$ with $\lambda_{\infty}^{(1)}(f) \in L^{\varphi}(\Omega)$. Since

$$
\left|f_{n}\right| \leq M_{n-1}(f)+\lambda_{n-1}^{(1)}(f),
$$

by the second inequality of (5.19), we have

$$
\|f\|_{P_{\varphi}(\Omega)} \lesssim\|f\|_{H_{\varphi}^{M}(\Omega)}+\left\|\lambda_{\infty}^{(1)}(f)\right\|_{L^{\varphi}(\Omega)} \lesssim\|f\|_{Q_{\varphi}(\Omega)} .
$$

Thus, we have $f=\left(f_{n}\right)_{n \in \mathbb{Z}_{+}} \in P_{\varphi}(\Omega)$. Then, by the definition of $P_{\varphi}(\Omega)$ (see Definition 2.1), we know that there exists an optimal control $\left(\lambda_{n}^{(2)}(f)\right)_{n \in \mathbb{Z}_{+}}$such that, for any $n \in \mathbb{N},\left|f_{n}\right| \leq \lambda_{n-1}^{(2)}(f)$ and $\lambda_{\infty}^{(2)}(f) \in L^{\varphi}(\Omega)$. Notice that, for any $n \in \mathbb{N}$,

$$
S_{n}(f) \leq S_{n-1}(f)+2 \lambda_{n-1}^{(2)}(f) .
$$

Using the first inequality of (5.19), we obtain the second inequality of (5.21).

Further, assume that $\left\{\mathcal{F}_{n}\right\}_{n \in \mathbb{Z}_{+}}$is regular. Since $\varphi \in A_{\infty}(\Omega)$, from Lemma 2.8, it follows that $\varphi \in \mathbb{S}$. By this and (5.21), we know that $P_{\varphi}(\Omega)=Q_{\varphi}(\Omega)$. Let $f \in P_{\varphi}(\Omega)$. From Theorems 4.4 and 4.6, it follows that, for any given $r \in(0,1]$,

$$
\|f\|_{P_{\varphi}(\Omega)} \sim\|f\|_{H_{\mathrm{at}, r}^{\varphi, \infty}(\Omega)} \sim\|f\|_{H_{\varphi}^{M}(\Omega)} .
$$

Similarly, for any $f \in Q_{\varphi}(\Omega)$, we know that, for any given $r \in(0,1]$,

$$
\|f\|_{Q_{\varphi}(\Omega)} \sim\|f\|_{H_{\mathrm{at}, r}^{\varphi, \infty, S}(\Omega)} \sim\|f\|_{H_{\varphi}^{S}(\Omega)} .
$$

By the regularity condition of $\left\{\mathcal{F}_{n}\right\}_{n \in \mathbb{Z}_{+}}$, we find that, for any $n \in \mathbb{N},\left|d_{n} f\right|^{2} \leq R \mathbb{E}_{n-1}\left(\left|d_{n} f\right|^{2}\right.$ ) (see, for example, [77, Proposition 2.18]) and hence

$$
S_{n}(f) \leq R^{\frac{1}{2}} S_{n}(f) .
$$

Since $s_{n}(f) \in \mathcal{F}_{n-1}$ for any $n \in \mathbb{N}$, from the definition of $Q_{\varphi}(\Omega)$, we deduce that

$$
\|f\|_{Q_{\varphi}(\Omega)} \lesssim\|s(f)\|_{L^{\varphi}(\Omega)} \sim\|f\|_{H_{\varphi}^{s}(\Omega)} .
$$

Now (5.20) yields that

$$
\|f\|_{Q_{\varphi}(\Omega)} \sim\|f\|_{H_{\varphi}^{s}(\Omega),}
$$

which completes the proof of Theorem 5.7. 
Remark 5.8. (i) Let $p \in(0, \infty)$. If $\varphi(x, t):=t^{p}$ for any $x \in \Omega$ and $t \in(0, \infty)$, then Theorem 5.7 in this case becomes [77, Theorem 2.22].

(ii) Let $\Phi$ be an Orlicz function. Theorem 5.7 when $\varphi(x, t):=\Phi(t)$ for any $x \in \Omega$ and $t \in(0, \infty)$ was proved by Miyamoto et al. [52, Theorem 2.5 and Corollary 2.6]. But, the assumptions of [77, Theorems 2.11 and 2.12] require that $\varphi$ is of lower type $p_{\varphi}^{-}$and of upper type $p_{\varphi}^{+}$ satisfying $0<p_{\varphi}^{-} \leq p_{\varphi}^{+} \leq 1$. However, Theorem 5.7 only needs $p_{\varphi}^{+} \in(0,2)$ in $(5.16)$ and (5.17). Therefore, in this sense, Theorem 5.7 generalizes and improves [52, Theorem 2.5 and Corollary 2.6].

(iii) Let $w \in A_{\infty}(\Omega)$ be a special weight and $\Phi$ an Orlicz function with lower type $p_{\varphi}^{-}$and upper type $p_{\varphi}^{+}$satisfying $0<p_{\varphi}^{-} \leq p_{\varphi}^{+}<\infty$. Letting $\varphi(x, t):=w(x) \Phi(t)$ for any $x \in \Omega$ and $t \in(0, \infty)$, then Theorem 5.7 with such a $\varphi$ is completely new.

Now we are ready to generalize the well-known Burkholder-Davis-Gundy inequality. To this end, we shall need the Davis decomposition of the martingales from $H_{\varphi}^{S}(\Omega)$ and $H_{\varphi}^{M}(\Omega)$ and some additional notions.

Definition 5.9. Let $\varphi$ be a Musielak-Orlicz function. The martingale Musielak-Orlicz Hardy space $G_{\varphi}(\Omega)$ is defined by setting

$$
G_{\varphi}(\Omega):=\left\{f \in \mathcal{M}:\|f\|_{G_{\varphi}(\Omega)}:=\left\|\sum_{n \in \mathbb{Z}_{+}}\left|d_{n} f\right|\right\|_{L^{\varphi}(\Omega)}<\infty\right\} .
$$

Lemma 5.10. Let $\varphi$ be a Musielak-Orlicz function with uniformly lower type $p_{\varphi}^{-}$for some $p_{\varphi}^{-} \in$ $[1, \infty)$. If the Doob maximal operator $M$ is bounded on $L^{\varphi^{*}}(\Omega)$ and $f \in H_{\varphi}^{S}(\Omega)$, then there exist $h \in G_{\varphi}(\Omega)$ and $g \in Q_{\varphi}(\Omega)$ such that $f_{n}=h_{n}+g_{n}$ for any $n \in \mathbb{Z}_{+}$, and there exists a positive constant $C$, independent of $f$, such that

$$
\|h\|_{G_{\varphi}(\Omega)} \leq C\|f\|_{H_{\varphi}^{S}(\Omega)} \quad \text { and } \quad\|g\|_{Q_{\varphi}(\Omega)} \leq C\|f\|_{H_{\varphi}^{S}(\Omega)} .
$$

Proof. Let $f \in H_{\varphi}^{S}(\Omega)$. Suppose that $0=\lambda_{0} \leq \lambda_{1} \leq \cdots$ is an adapted sequence of functions such that, for any $n \in \mathbb{Z}_{+}$,

$$
S_{n}(f) \leq \lambda_{n} \quad \text { and } \quad \lambda_{\infty}:=\sup _{n \in \mathbb{Z}_{+}} \lambda_{n} \in L^{\varphi}(\Omega) .
$$

Clearly, for any $n \in \mathbb{N}$, we have

$$
d_{n} f=d_{n} f \mathbf{1}_{\left\{x \in \Omega: \lambda_{n}(x)>2 \lambda_{n-1}(x)\right\}}+d_{n} f \mathbf{1}_{\left\{x \in \Omega: \lambda_{n}(x) \leq 2 \lambda_{n-1}(x)\right\}} .
$$

For any $n \in \mathbb{N}$, let

$$
h_{n}:=\sum_{k=1}^{n}\left[d_{k} f \mathbf{1}_{\left\{x \in \Omega: \lambda_{k}(x)>2 \lambda_{k-1}(x)\right\}}-\mathbb{E}_{k-1}\left(d_{k} f \mathbf{1}_{\left\{x \in \Omega: \lambda_{k}(x)>2 \lambda_{k-1}(x)\right\}}\right)\right]
$$

and

$$
g_{n}:=\sum_{k=1}^{n}\left[d_{k} f \mathbf{1}_{\left\{x \in \Omega: \lambda_{k}(x) \leq 2 \lambda_{k-1}(x)\right\}}-\mathbb{E}_{k-1}\left(d_{k} f \mathbf{1}_{\left\{x \in \Omega: \lambda_{k}(x) \leq 2 \lambda_{k-1}(x)\right\}}\right)\right] .
$$


Then, for any $n \in \mathbb{N}, f_{n}=h_{n}+g_{n}$. For any $k \in \mathbb{Z}$, on the set $\left\{x \in \Omega: \lambda_{k}(x)>2 \lambda_{k-1}(x)\right\}$, we have $\lambda_{k}<2\left(\lambda_{k}-\lambda_{k-1}\right)$, henceforth

$$
\left.\left|d_{k} f\right| \mathbf{1}_{\{x \in \Omega:} \lambda_{k}(x)>2 \lambda_{k-1}(x)\right\} \leq \lambda_{k} \mathbf{1}_{\left\{x \in \Omega: \lambda_{k}(x)>2 \lambda_{k-1}(x)\right\}} \leq 2\left(\lambda_{k}-\lambda_{k-1}\right) .
$$

Thus, we conclude that, for any $n \in \mathbb{N}$,

$$
\sum_{k=1}^{n}\left|d_{k} h\right| \leq 2 \lambda_{n}+2 \sum_{k=1}^{n} \mathbb{E}_{k-1}\left(\lambda_{k}-\lambda_{k-1}\right) .
$$

From this and Theorem 3.5, it follows that

$$
\|h\|_{G_{\varphi}(\Omega)} \lesssim\left\|\lambda_{\infty}\right\|_{L^{\varphi}(\Omega)} .
$$

On another hand, for any $k \in \mathbb{N}$, we have

$$
\left|d_{k} f\right| \mathbf{1}_{\left\{x \in \Omega: \lambda_{k}(x) \leq 2 \lambda_{k-1}(x)\right\}} \leq \lambda_{k} \mathbf{1}_{\left\{x \in \Omega: \lambda_{k}(x) \leq 2 \lambda_{k-1}(x)\right\}} \leq 2 \lambda_{k-1},
$$

which implies that

$$
\left|d_{k} g\right| \leq 4 \lambda_{k-1} .
$$

Combining this and (5.22), we conclude that, for any $n \in \mathbb{N}$,

$$
\begin{aligned}
S_{n}(g) & \leq S_{n-1}(g)+\left|d_{n} g\right| \leq S_{n-1}(f)+S_{n-1}(h)+4 \lambda_{n-1} \\
& \leq \lambda_{n-1}+2 \lambda_{n-1}+2 \sum_{k=1}^{n-1} \mathbb{E}_{k-1}\left(\lambda_{k}-\lambda_{k-1}\right)+4 \lambda_{n-1} .
\end{aligned}
$$

From this and Theorem 3.5, it follows that

$$
\|g\|_{Q_{\varphi}(\Omega)} \lesssim\left\|\lambda_{\infty}\right\|_{L^{\varphi}(\Omega)}
$$

For any $n \in \mathbb{Z}_{+}$, letting $\lambda_{n}:=S_{n}(f)$, we then obtain the desired conclusion. This finishes the proof of Lemma 5.10.

Using Theorem 3.5, we also obtain the Davis decomposition of $H_{\varphi}^{M}(\Omega)$, whose proof is similar to that of Lemma 5.10, the details being omitted.

Lemma 5.11. Let $\varphi$ be a Musielak-Orlicz function with uniformly lower type $p_{\varphi}^{-}$for some $p_{\varphi}^{-} \in$ $[1, \infty)$. If the Doob maximal operator $M$ is bounded on $L^{\varphi^{*}}(\Omega)$ and $f \in H_{\varphi}^{M}(\Omega)$, then there exist $h \in G_{\varphi}(\Omega)$ and $g \in P_{\varphi}(\Omega)$ such that $f_{n}=h_{n}+g_{n}$ for any $n \in \mathbb{Z}_{+}$, and there exists a positive constant $C$, independent of $f$, such that

$$
\|h\|_{G_{\varphi}(\Omega)} \leq C\|f\|_{H_{\varphi}^{M}(\Omega)} \quad \text { and } \quad\|g\|_{P_{\varphi}(\Omega)} \leq C\|f\|_{H_{\varphi}^{M}(\Omega)} .
$$

The generalization of Burkholder-Davis-Gundy inequalities reads as follows. 
Theorem 5.12. Let $\varphi \in A_{\infty}(\Omega) \cap \mathbb{S}^{-}$be a Musielak-Orlicz function with uniformly lower type $p_{\varphi}^{-}$ and uniformly upper type $p_{\varphi}^{+}$satisfying $1<p_{\varphi}^{-} \leq p_{\varphi}^{+}<\infty$. If the Doob maximal operator $M$ is bounded on $L^{\varphi^{*}}(\Omega)$, then there exists a positive constant $C$ such that, for any martingale $f \in \mathcal{M}$,

$$
\frac{1}{C}\|f\|_{H_{\varphi}^{S}(\Omega)} \leq\|f\|_{H_{\varphi}^{M}(\Omega)} \leq C\|f\|_{H_{\varphi}^{S}(\Omega)} .
$$

Proof. First we prove the second inequality of (5.23). Let $f \in H_{\varphi}^{S}(\Omega)$. By Lemma 5.10, we know that there exist $h \in G_{\varphi}(\Omega)$ and $g \in Q_{\varphi}(\Omega)$ such that, for any $n \in \mathbb{Z}_{+}, f_{n}=h_{n}+g_{n}$ and

$$
\|h\|_{G_{\varphi}(\Omega)} \lesssim\|f\|_{H_{\varphi}^{S}(\Omega)}, \quad\|g\|_{Q_{\varphi}(\Omega)} \lesssim\|f\|_{H_{\varphi}^{S}(\Omega)} .
$$

It is easy to show that $M(h) \leq \sum_{n \in \mathbb{Z}_{+}}\left|d_{n} h\right|$. From this, it follows that

$$
\|h\|_{H_{\varphi}^{M}(\Omega)} \leq\|h\|_{G_{\varphi}(\Omega)} .
$$

Using this, (5.19) and (5.24), we conclude that

$$
\|f\|_{H_{\varphi}^{M}(\Omega)} \leq\|h\|_{H_{\varphi}^{M}(\Omega)}+\|g\|_{H_{\varphi}^{M}(\Omega)} \lesssim\|h\|_{G_{\varphi}(\Omega)}+\|g\|_{Q_{\varphi}(\Omega)} \lesssim\|f\|_{H_{\varphi}^{S}(\Omega)} .
$$

The first inequality of (5.23) can be proved in the same way. This finishes the proof of Theorem 5.12 .

Remark 5.13. If, in Lemmas 5.10 and 5.11 and Theorem 5.12, the boundedness of the Doob maximal operator on $L^{\varphi^{*}}(\Omega)$ is replaced by the condition that $\varphi^{*} \in A_{\infty}(\Omega)$ satisfies (3.3), then, from Corollary 3.4, we deduce that Lemmas 5.10 and 5.11 and Theorem 5.12 still hold true.

Now, we turn to Burkholder-Davis-Gundy inequalities for the endpoint case: the uniformly lower type index $p_{\varphi}^{-}=1$. To this end, we first prove the following theorem, which is a generalization of [10, Theorem 3.2].

Theorem 5.14. Let $\Phi:[0, \infty) \rightarrow[0, \infty)$ be an Orlicz function and $w$ a weight. Let $\varphi(x, t)=$ $w(x) \Phi(t)$ for any $x \in \Omega$ and $t \in(0, \infty)$. If $w \in A_{1}(\Omega)$ and $\varphi$ is of uniformly lower type 1 and of uniformly upper type $p_{\varphi}^{+}$for some $p_{\varphi}^{+} \in[1, \infty)$, then there exists a positive constant $C$ such that, for any sequence $\left(g_{k}\right)_{k \in \mathbb{Z}_{+}}$of non-negative $\mathcal{F}$ measurable functions,

$$
\left\|\sum_{k \in \mathbb{Z}_{+}} \mathbb{E}_{k}\left(g_{k}\right)\right\|_{L^{\varphi}(\Omega)} \leq C\left\|\sum_{k \in \mathbb{Z}_{+}} g_{k}\right\|_{L^{\varphi}(\Omega)} .
$$

Proof. Let $\widehat{d \mathbb{P}}:=w d \mathbb{P}$. Denote the expectation and the conditional expectation operators related to $\widehat{\mathbb{P}}$, respectively, by $\widehat{\mathbb{E}}$ and $\widehat{\mathbb{E}}_{n}$. Since $w \in L^{1}(\Omega)$, we may assume that $\widehat{\mathbb{P}}$ is a probability measure. Then $\left(\Omega, \mathcal{F},\left\{\mathcal{F}_{n}\right\}_{n \in \mathbb{Z}_{+}}, \widehat{\mathbb{P}}\right)$ is a probability space. Combining this and [10, Theorem 3.2], we obtain

$$
\widehat{\mathbb{E}} \Phi\left(\sum_{n=1}^{\infty} \widehat{\mathbb{E}}_{n}\left(g_{n}\right)\right) \lesssim \widehat{\mathbb{E}} \Phi\left(\sum_{n=1}^{\infty} g_{n}\right) .
$$


For any $\mathcal{F}$ measurable function $f$, by [50, Proposition 6.1.7] (or [17, Section 4]), we know that, for any $n \in \mathbb{Z}_{+}$,

$$
\widehat{\mathbb{E}}_{n}(f)=\frac{1}{w_{n}} \mathbb{E}_{n}(f w) .
$$

By this and $w \in A_{1}(\Omega)$, we find that, for any $n \in \mathbb{Z}_{+}$,

$$
\widehat{\mathbb{E}}_{n}(f)=\mathbb{E}_{n}\left(\frac{1}{w_{n}} f w\right) \gtrsim \mathbb{E}_{n}(f) .
$$

From this and (5.25), it follows that

$$
\widehat{\mathbb{E}} \Phi\left(\sum_{n=1}^{\infty} \mathbb{E}_{n}\left(g_{n}\right)\right) \lesssim \widehat{\mathbb{E}} \Phi\left(\sum_{n=1}^{\infty} g_{n}\right),
$$

which implies that

$$
\int_{\Omega} \varphi\left(x, \sum_{n=1}^{\infty} \mathbb{E}_{n}\left(g_{n}\right)(x)\right) d \mathbb{P} \lesssim \int_{\Omega} \varphi\left(x, \sum_{n=1}^{\infty} g_{n}(x)\right) d \mathbb{P} .
$$

This finishes the proof of Theorem 5.14.

Remark 5.15. In Theorem 5.14, the uniformly lower type 1 and the uniformly upper type $p^{+} \in$ $[1, \infty)$ properties of $\varphi$ can be replaced by the condition that $\Phi$ is convex and $\Phi \in \Delta_{2}$ (that is, $\Phi(2 t) \leq K \Phi(t)$ for any $t \in(0, \infty))$. Indeed, if $\Phi$ is of lower type 1, then, by [28, Proposition 2.3] (see also [15, Lemma 2.2]), we know that $\Phi$ is equivalent to a convex function. On another hand, it is clear that $\Phi \in \Delta_{2}$ if and only if $\varphi$ is of uniformly upper type $p^{+} \in[1, \infty)$.

Using Theorem 5.14 and Remark 5.15, we can prove the following theorem in the way same as the proof of Theorem 5.12, the details being omitted.

Theorem 5.16. Let $\Phi:[0, \infty) \rightarrow[0, \infty)$ be an Orlicz function and $w$ a special weight. Let $\varphi(x, t):=w(x) \Phi(t)$ for any $x \in \Omega$ and $t \in(0, \infty)$. If $\varphi$ is of uniformly lower type 1 and of uniformly upper type $p_{\varphi}^{+}$for some $p_{\varphi}^{+} \in[1, \infty)$ and $w \in A_{1}(\Omega)$, then there exists a positive constant $C$ such that, for any martingale $f \in \mathcal{M}$,

$$
\frac{1}{C}\|f\|_{H_{\varphi}^{S}(\Omega)} \leq\|f\|_{H_{\varphi}^{M}(\Omega)} \leq C\|f\|_{H_{\varphi}^{S}(\Omega)} .
$$

Now, we compare Theorems 5.12 and 5.16 with Bonami and Lépingle [7, Theorem 1]. To this end, we need the following notion, which was first introduced by Doléans-Dade et al. [17].

Definition 5.17. Let $p \in(1, \infty)$ and $w$ be a special weight. Then $w$ is said to satisfy an $\widehat{A}_{p}(\Omega)$ condition, denoted by $w \in \widehat{A}_{p}(\Omega)$, if there exists a positive constant $C$ such that, for any $n \in \mathbb{Z}_{+}$,

$$
\frac{1}{w_{n}}\left[\widehat{\mathbb{E}}_{n}\left(w^{\frac{1}{p-1}}\right)\right]^{p-1} \leq C
$$

Then $w$ is said to satisfy $\widehat{A}_{\infty}(\Omega)$ if $w \in \widehat{A}_{p}(\Omega)$ for some $p \in(1, \infty)$. 
Remark 5.18. Recall that Bonami and Lépingle [7, Theorem 1] proved that, if $w \in \widehat{A_{\infty}}(\Omega) \cap \mathbb{S}^{-}$, then the Burkholder-Davis-Gundy inequality holds true in the weighted Orlicz case. Now we claim that $\widehat{A}_{\infty}(\Omega) \cap \mathbb{S}^{-}$is slightly stronger than the condition $A_{\infty}(\Omega) \cap \mathbb{S}^{-}$. Indeed, let $w \in \widehat{A}_{\infty}(\Omega) \cap$ $\mathbb{S}^{-}$, then there exists an index $p \in(1, \infty)$ such that $w \in \widehat{A_{p}}(\Omega)$. From [7, p. 298], we deduce that $w \in \widehat{A}_{p}(\Omega)$ is equivalent to

$$
\mathbb{E}_{n}\left(w^{\frac{p}{p-1}}\right) \leq C_{p} w_{n}^{\frac{p}{p-1}}, \quad \forall n \in \mathbb{Z}_{+}
$$

Combining this, $w \in \mathbb{S}^{-}$and [17, Proposition 5], we know that there exists an index $q \in(1, \infty)$ such that $w \in A_{q}(\Omega)$. From this, it follows that $w \in A_{\infty}(\Omega) \cap \mathbb{S}^{-}$. This proves the above claim (see also [50, Proposition 6.1.8 and Remark 6.6.9]).

Thus, when $\varphi(x, t):=w(x) \Phi(t)$ for any $x \in \Omega$ and $t \in(0, \infty)$, the assumption on the weight of Theorem 5.12 is slightly weaker than the assumption on the weight of [7, Theorem 1]. However, Theorems 5.12 and 5.16 can not cover [7, Theorem 1], because Theorem 5.12 needs that the Doob maximal operator $M$ is bounded on $L^{\varphi^{*}}(\Omega)$ and Theorem 5.16 needs $w \in A_{1}(\Omega)$. If $\varphi(x, t):=t^{p}$ for any $x \in \Omega$ and $t \in(0, \infty)$, with $p \in[1, \infty)$, then Theorems 5.12 and 5.16 become the classical Burkholder-Davis-Gundy inequality (see [10]).

The following corollary follows immediately from Lemma 2.8 and Theorems 4.1, 4.4, 4.6 and 5.7 .

Corollary 5.19. Let $\varphi$ be a Musielak-Orlicz function with uniformly lower type $p_{\varphi}^{-}$and uniformly upper type $p_{\varphi}^{+}$satisfying $0<p_{\varphi}^{-} \leq p_{\varphi}^{+}<\infty$. If $\varphi \in A_{\infty}(\Omega)$ and $\left\{\mathcal{F}_{n}\right\}_{n \in \mathbb{Z}_{+}}$is regular, then, for any given $r \in(0,1], H_{\varphi}(\Omega)=H_{\mathrm{at}, r}^{\varphi, \infty}(\Omega)$ with equivalent quasi-norms, here $T$ stands for any one of operators $M, s$ and $S$, and $H_{\varphi}(\Omega)$ denotes any one of five Musielak-Orlicz martingale Hardy spaces $H_{\varphi}^{M}(\Omega), P_{\varphi}(\Omega), H_{\varphi}^{s}(\Omega), H_{\varphi}^{S}(\Omega)$ and $Q_{\varphi}(\Omega)$.

Definition 5.20. For any $f:=\left(f_{n}\right)_{n \in \mathbb{Z}_{+}} \in \mathcal{M}$, the martingale transform $\mathcal{T}$ is defined by setting, for any $n \in \mathbb{N}$,

$$
(\mathcal{T} f)_{n}:=\sum_{k=1}^{n} v_{k-1} d_{k} f \text { and }(\mathcal{T} f)_{0}:=0,
$$

where, for any $k \in \mathbb{Z}_{+}, v_{k}$ is $\mathcal{F}_{k}$ measurable and $\left\|v_{k}\right\|_{L^{\infty}(\Omega)} \leq 1$.

Now we turn to the boundedness of the martingale transform on $L^{\varphi}(\Omega)$.

Theorem 5.21. Let $\varphi \in A_{\infty}(\Omega)$ be a Musielak-Orlicz function satisfying (3.2). If the stochastic basis $\left\{\mathcal{F}_{n}\right\}_{n \in \mathbb{Z}_{+}}$is regular, then the martingale transform $\mathcal{T}$ is bounded on $L^{\varphi}(\Omega)$.

Proof. Since $\left\|v_{k}\right\|_{L^{\infty}(\Omega)} \leq 1$, we have $S(\mathcal{T} f) \leq S(f) \mathbb{P}$-almost everywhere. From this, Theorems 5.7 and 3.2 , it follows that

$$
\|\mathcal{T} f\|_{L^{\varphi}(\Omega)} \leq\|M(\mathcal{T} f)\|_{L^{\varphi}(\Omega)} \lesssim\|S(f)\|_{L^{\varphi}(\Omega)} \lesssim\|M(f)\|_{L^{\varphi}(\Omega)} \lesssim\|f\|_{L^{\varphi}(\Omega)},
$$

which completes the proof of Theorem 5.21. 


\section{Walsh system and Fejér means}

Let us investigate the dyadic martingales. Namely, let $\Omega:=[0,1), \mathbb{P}$ be the Lebesgue measure and $\mathcal{F}$ the set of all Lebesgue measurable sets. By a dyadic interval, we mean one of the form $\left[k 2^{-n},(k+1) 2^{-n}\right)$ for some $k, n \in \mathbb{Z}_{+}$and $0 \leq k<2^{n}$. For any given $n \in \mathbb{Z}_{+}$and $x \in[0,1)$, denote by $I_{n}(x)$ the dyadic interval of length $2^{-n}$ which contains $x$. For any $n \in \mathbb{Z}_{+}$, the $\sigma$-algebras generated by the dyadic intervals $\left\{I_{n}(x): x \in[0,1)\right\}$ is denoted by $\mathcal{F}_{n}$. It is easy to see that $\left(\mathcal{F}_{n}\right)_{n \in \mathbb{Z}_{+}}$ is regular and increasing.

For any $n \in \mathbb{Z}_{+}$, the Rademacher function $r_{n}$ is defined by setting

$$
r(x):= \begin{cases}1 & \text { if } x \in\left[0, \frac{1}{2}\right) \\ -1 & \text { if } x \in\left[\frac{1}{2}, 1\right)\end{cases}
$$

and, for any $x \in[0,1)$,

$$
r_{n}(x):=r\left(2^{n} x\right)
$$

It is clear that, for any $n \in \mathbb{Z}_{+}, r_{n}$ is $\mathcal{F}_{n+1}$ measurable. The product system generated by the Rademacher functions is the Walsh system

$$
w_{n}:=\prod_{k \in \mathbb{Z}_{+}} r_{k}^{n_{k}}, \quad \forall n \in \mathbb{Z}_{+},
$$

where

$$
n=\sum_{k \in \mathbb{Z}_{+}} n_{k} 2^{k}, \quad n_{k} \in\{0,1\}
$$

Recall (see Fine [21]) that the Walsh-Dirichlet kernels, defined by setting, for any $n \in \mathbb{N}$,

$$
D_{n}:=\sum_{k=0}^{n-1} w_{k},
$$

satisfy

$$
D_{2^{k}}(x)=\left\{\begin{array}{ll}
2^{k} & \text { if } x \in\left[0,2^{-k}\right), \\
0 & \text { if } x \in\left[2^{-k}, 1\right),
\end{array} \quad \forall k \in \mathbb{Z}_{+} .\right.
$$

If $f \in L^{1}[0,1)$, for any $n \in \mathbb{Z}_{+}$, the number $\widehat{f}(n):=\mathbb{E}\left(f w_{n}\right)$ is called the $n$th Walsh-Fourier coefficient of $f$. We can extend this definition to martingales as follows. If $f:=\left(f_{k}\right)_{k \in \mathbb{Z}_{+}}$is a martingale, then, for any $n \in \mathbb{Z}_{+}$, let

$$
\widehat{f}(n):=\lim _{k \rightarrow \infty} \mathbb{E}\left(f_{k} w_{n}\right)
$$

Since, for any $k, n \in \mathbb{Z}_{+}$and $n<2^{k}, w_{n}$ is $\mathcal{F}_{k}$ measurable, it can immediately be seen that the above limit does exist. Recall that, if $f \in L^{1}[0,1)$, then $\mathbb{E}_{k} f \rightarrow f$ in the $L^{1}[0,1)$-norm, as $k \rightarrow \infty$, hence, for any $n \in \mathbb{Z}_{+}$,

$$
\widehat{f}(n)=\lim _{k \rightarrow \infty} \mathbb{E}\left(\left(\mathbb{E}_{k} f\right) w_{n}\right) .
$$


Thus, the Walsh-Fourier coefficients of $f \in L^{1}[0,1)$ are the same as the ones of the martingale $\left(\mathbb{E}_{k} f\right)_{k \in \mathbb{Z}_{+}}$obtained from $f$.

For any $n \in \mathbb{N}$, denote by $s_{n} f$ the $n$th partial sum of the Walsh-Fourier series of a martingale $f$, namely,

$$
s_{n} f:=\sum_{k=0}^{n-1} \widehat{f}(k) w_{k} .
$$

If $f \in L^{1}[0,1)$, then, for any $n \in \mathbb{N}$,

$$
s_{n} f(x)=\int_{0}^{1} f(t) D_{n}(x+t) d t,
$$

where $\dot{+}$ denotes the dyadic addition (see, for example, Schipp et al. [68] or Golubov et al. [25]). It is easy to see that, for any $n \in \mathbb{Z}_{+}$,

$$
s_{2^{n}} f=f_{n}
$$

and hence, by the martingale convergence theorem [64, Theorem 1.34], we know that, for any $p \in[1, \infty)$ and $f \in L^{p}[0,1)$,

$$
\lim _{n \rightarrow \infty} s_{2^{n}} f=f \quad \text { in the } L^{p}[0,1) \text {-norm. }
$$

This result was generalized by Schipp et al. [68, Theorem 4.1]. More precisely, they proved that, for any $p \in(1, \infty)$ and $f \in L^{p}[0,1)$,

$$
\lim _{n \rightarrow \infty} s_{n} f=f \quad \text { in the } L^{p}[0,1) \text {-norm. }
$$

Using the method of martingale transforms, we generalize [68, Theorem 4.1] to $L^{\varphi}[0,1)$.

Theorem 6.1. Let $\varphi \in A_{\infty}[0,1)$ be a Musielak-Orlicz function satisfying (3.2). Then there exists a positive constant $C$ such that, for any $f \in L^{\varphi}[0,1)$,

$$
\sup _{n \in \mathbb{N}}\left\|s_{n} f\right\|_{L^{\varphi}[0,1)} \leq C\|f\|_{L^{\varphi}[0,1)} .
$$

Proof. It was proved by Schipp et al. [68, p. 95] that, for any $n \in \mathbb{N}$,

$$
s_{n} f=w_{n} \mathcal{T}_{0}\left(f w_{n}\right),
$$

where

$$
\mathcal{T}_{0} f:=\sum_{k=1}^{\infty} n_{k-1} d_{k} f
$$

and the binary coefficients $n_{k}$ are defined as in (6.1). Combining this, Theorem 5.21 and $\left|w_{n}\right|=1$ for any $n \in \mathbb{N}$, we know that, for any $n \in \mathbb{N}$,

$$
\left\|s_{n} f\right\|_{L^{\varphi}[0,1)}=\left\|\mathcal{T}_{0}\left(f w_{n}\right)\right\|_{L^{\varphi}[0,1)} \lesssim\left\|f w_{n}\right\|_{L^{\varphi}[0,1)} \lesssim\|f\|_{L^{\varphi}[0,1)},
$$

which completes the proof of Theorem 6.1. 
Corollary 6.2. Let $\varphi \in A_{\infty}[0,1)$ be a Musielak-Orlicz function satisfying (3.2). Then, for any $f \in L^{\varphi}[0,1)$,

$$
\lim _{n \rightarrow \infty} s_{n} f=f \quad \text { in the } L^{\varphi}[0,1) \text {-norm. }
$$

Proof. It is enough to show that the Walsh polynomials are dense in $L^{\varphi}[0,1)$. Indeed, by $(6.2), f_{n}$ is a Walsh polynomial. By Theorem 5.7, we know that, for any $n \in \mathbb{Z}_{+}$,

$$
\int_{0}^{1} \varphi\left(x,\left|f(x)-f_{n}(x)\right|\right) d x \leq \int_{0}^{1} \varphi\left(x, M\left(f-f_{n}\right)(x)\right) d x \sim \int_{0}^{1} \varphi\left(x, S\left(f-f_{n}\right)(x)\right) d x .
$$

For almost every $x \in[0,1)$, we have

$$
\lim _{n \rightarrow \infty} S\left(f-f_{n}\right)(x)=\lim _{n \rightarrow \infty}\left(\sum_{k=n+1}^{\infty}\left|d_{k+1} f\right|^{2}\right)^{1 / 2}=0 .
$$

By this and the facts that $S\left(f-f_{n}\right)(x) \leq S(f)(x)$ and $\varphi$ is of uniformly lower type $p_{\varphi}^{-}$, we know that, for almost every $x \in[0,1)$,

$$
\lim _{n \rightarrow \infty} \varphi\left(x, S\left(f-f_{n}\right)(x)\right) \lesssim \lim _{n \rightarrow \infty}\left[\frac{S\left(f-f_{n}\right)(x)}{S(f)(x)}\right]^{p_{\varphi}^{-}} \varphi(x, S(f)(x))=0 .
$$

From this and the Lebesgue dominated convergence theorem, it follows that

$$
\lim _{n \rightarrow \infty} \int_{0}^{1} \varphi\left(x, S\left(f-f_{n}\right)(x)\right) d x=0 .
$$

Combining this and (6.4), we have

$$
\lim _{n \rightarrow \infty}\left\|f-f_{n}\right\|_{L^{\varphi}[0,1)}=0 .
$$

This proves that the Walsh polynomials are dense in $L^{\varphi}[0,1)$. The corollary follows from Theorem 6.1 with the usual density argument. This finishes the proof of Corollary 6.2.

If we do not suppose that $\varphi \in A_{\infty}[0,1)$ satisfies (3.2), then (6.3) is not true. However, to generalize the convergence result, in this case, we can consider a summability method. Recall that, for any $n \in \mathbb{N}$, the Fejér means $\sigma_{n} f$ of the Walsh-Fourier series of a martingale $f$ is defined in (1.3). Of course, $\left\{\sigma_{n} f\right\}_{n \in \mathbb{N}}$ has better convergence properties than $\left\{s_{n} f\right\}_{n \in \mathbb{N}}$. It is easy to show that, for any $f \in L^{1}[0,1)$ and $n \in \mathbb{N}$,

$$
\sigma_{n} f(x)=\int_{0}^{1} f(t) K_{n}(x \dot{+} t) d t,
$$

where, for any $n \in \mathbb{N}$, the Walsh-Fejér kernel $K_{n}$ is defined by setting

$$
K_{n}:=\frac{1}{n} \sum_{k=1}^{n} D_{k}
$$


It is known (see Fine [21] or Schipp et al. [68, Theorem 1.16]) that, for any $n \in \mathbb{N}$ and $x \in[0,1$ ),

$$
\left|K_{n}(x)\right| \leq \sum_{j=0}^{N-1} 2^{j-N} \sum_{i=j}^{N-1}\left[D_{2^{i}}(x)+D_{2^{i}}\left(x \dot{+} 2^{-j-1}\right)\right]
$$

and

$$
K_{2^{n}}(x)=\frac{1}{2}\left[2^{-n} D_{2^{n}}(x)+\sum_{j=0}^{n} 2^{j-n} D_{2^{n}}\left(x \dot{+} 2^{-j-1}\right)\right],
$$

where $N$ is a positive integer such that $2^{N-1} \leq n<2^{N}$.

\section{The maximal Fejér operator on $H_{\varphi}[0,1)$}

We have proved in Theorem 5.7 that, if $\varphi \in A_{\infty}(\Omega)$ is a Musielak-Orlicz function with uniformly lower type $p_{\varphi}^{-}$and uniformly upper type $p_{\varphi}^{+}$and $\left\{\mathcal{F}_{n}\right\}_{n \in \mathbb{Z}_{+}}$regular, then all the five martingale Musielak-Orlicz Hardy spaces are equivalent. In this section, we consider the dyadic $\sigma$-algebras, so $\left\{\mathcal{F}_{n}\right\}_{n \in \mathbb{Z}_{+}}$is regular. If we deal with Musielak-Orlicz Hardy spaces, we always suppose the other conditions, namely, $\varphi \in A_{\infty}[0,1)$ is a Musielak-Orlicz function with uniformly lower type $p_{\varphi}^{-}$and uniformly upper type $p_{\varphi}^{+}$. Denote by $H_{\varphi}[0,1)$ one of the five martingale Musielak-Orlicz Hardy spaces.

In this section, we prove the boundedness of $\sigma_{*}$ from $H_{\varphi}[0,1)$ to $L^{\varphi}[0,1)$. It is known that, for any $f \in L^{\varphi}[0,1)$, the Doob maximal operator $M(f)$ can be written as

$$
M(f)(x)=\sup _{x \in I} \frac{1}{\mathbb{P}(I)}\left|\int_{I} f d \mathbb{P}\right|
$$

where $I$ is a dyadic interval and $\mathbb{P}$ a Lebesgue measure. Motivating by this and the kernel functions (6.5) and (6.6), we give two other dyadic maximal functions, which are originally introduced by Jiao et al. [40]. Let $f \in L^{\varphi}[0,1), r \in(0, \infty)$ and $v$ be a bounded measure. For any $n \in \mathbb{N}$ and $x \in[0,1)$, let

$$
U_{v, r, n}(f)(x):=\sup _{I \ni x} \sum_{j=0}^{n-1} 2^{(j-n) r} \frac{1}{v\left(I+2^{-j-1}\right)}\left|\int_{I+2^{-j-1}} f d v\right|
$$

and

$$
V_{v, r, n}(f)(x):=\sup _{I \ni x} \sum_{j=0}^{n-1} \sum_{i=j}^{n-1} 2^{(j-n) r} 2^{(i-n) r} 2^{n-i} \frac{1}{v\left(I+\left[2^{-j-1}, 2^{-j-1}+2^{-i}\right)\right)}\left|\int_{I+\left[2^{-j-1}, 2^{-j-1}+2^{-i}\right)} f d v\right|,
$$

where the suprema are taken over all dyadic intervals $I$ with length $2^{-n}$, which contain $x$. For $v=\mathbb{P}$, we write simply $U_{r, n}$ and $V_{r, n}$. The proof of the following theorem is similar to that of [40, Theorems 7.7 and 7.10] and the details are omitted.

Theorem 7.1. Let $p \in(1, \infty]$ and $v$ be a bounded measure. If $r \in(0, \infty)$, then there exists a positive constant $C$ such that, for any $n \in \mathbb{N}$ and $f \in L^{p}([0,1), d v)$,

$$
\left\|U_{v, r, n}(f)\right\|_{L^{p}([0,1), d v)} \leq C\|f\|_{L^{p}([0,1), d v)} .
$$


If $r \in\left(\frac{1}{2}, 1\right]$, then there exists a positive constant $C$ such that, for any $n \in \mathbb{N}$ and $f \in L^{p}([0,1), d v)$,

$$
\left\|V_{v, r, n}(f)\right\|_{L^{p}([0,1), d v)} \leq C\|f\|_{L^{p}([0,1), d v)} .
$$

Let $p \in(1, \infty)$. Suppose that $w \in A_{p}[0,1)$ and $v$ is the measure generated by $w$, namely, $d v=w d \mathbb{P}$. For any dyadic interval $I$, by $w \in A_{p}[0,1)$, we have

$$
\begin{aligned}
\frac{1}{\mathbb{P}(I)} \int_{I}|f| d \mathbb{P} & \leq \frac{1}{\mathbb{P}(I)}\left(\int_{I}|f|^{p} w d \mathbb{P}\right)^{1 / p}\left[\int_{I} w^{-1 /(p-1)} d \mathbb{P}\right]^{(p-1) / p} \\
& \leq\left[\frac{1}{v(I)} \int_{I}|f|^{p} d v\right]^{1 / p}\left\{\frac{1}{\mathbb{P}(I)} \int_{I} w d \mathbb{P}\left[\frac{1}{\mathbb{P}(I)} \int_{I} w^{-1 /(p-1)} d \mathbb{P}\right]^{p-1}\right\}^{1 / p} \\
& \lesssim\left[\frac{1}{v(I)} \int_{I}|f|^{p} d v\right]^{1 / p} .
\end{aligned}
$$

Using this, we can prove the next theorem in the usual way (see, for example, Strömberg and Torchinsky [72]), the proof being omitted.

Theorem 7.2. Let $p \in(1, \infty)$ and $w \in A_{p}[0,1)$. If $r \in(0, \infty)$, then there exists a positive constant $C$ such that, for any $n \in \mathbb{N}$ and $f \in L^{p}([0,1), w d \mathbb{P})$,

$$
\left\|U_{r, n}(f)\right\|_{L^{p}([0,1), w d \lambda)} \leq C\|f\|_{L^{p}([0,1), w d \mathbb{P})} .
$$

If $r \in\left(\frac{1}{2}, 1\right]$, then there exists a positive constant $C$ such that, for any $n \in \mathbb{N}$ and $f \in L^{p}([0,1), w d \mathbb{P})$,

$$
\left\|V_{r, n}(f)\right\|_{L^{p}([0,1), w d \lambda)} \leq C\|f\|_{L^{p}([0,1), w d \mathbb{P})} .
$$

Theorem 7.3. Let $\varphi \in A_{\infty}[0,1)$ be a Musielak-Orlicz function with uniformly lower type $p_{\varphi}^{-}$and uniformly upper type $p_{\varphi}^{+}$satisfying (3.2). If $r \in(0, \infty)$, then there exists a positive constant $C$ such that, for any $n \in \mathbb{N}$ and $f \in L^{\varphi}[0,1)$,

$$
\left\|U_{r, n}(f)\right\|_{L^{\varphi}[0,1)} \leq C\|f\|_{L^{\varphi}[0,1)} .
$$

Moreover, if $r \in\left(\frac{1}{2}, 1\right]$, then there exists a positive constant $C$ such that, for any $n \in \mathbb{N}$ and $f \in L^{\varphi}[0,1)$,

$$
\left\|V_{r, n}(f)\right\|_{L^{\varphi}[0,1)} \leq C\|f\|_{L^{\varphi}[0,1)} .
$$

Proof. Using Theorems 3.1 and 7.2, we can show Theorem 7.3 by the way same as the proof of Theorem 3.2, the details being omitted. This finishes the proof of Theorem 7.3.

Next we give a sufficient condition for a $\sigma$-sublinear operator to be bounded from $H_{\varphi}[0,1)$ to $L^{\varphi}[0,1)$.

Theorem 7.4. Let $\varphi \in A_{\infty}[0,1)$ be a Musielak-Orlicz function with uniformly lower type $p_{\varphi}^{-}$and uniformly upper type $p_{\varphi}^{+}$. Suppose that the Doob maximal operator $M$ is bounded on $L_{1 / r}^{\varphi_{1}^{*}}[0,1)$ for 
some $r \in\left(0, \min \left\{1, \wedge p_{\varphi}^{-}\right\}\right]$. Suppose further that the $\sigma$-sublinear operator $T: L^{\infty}[0,1) \rightarrow L^{\infty}[0,1)$ is bounded and

$$
\left\|\sum_{k \in \mathbb{Z}}\left(\mu^{k}\right)^{r}\left|T\left(a^{k}\right)\right|^{r} \mathbf{1}_{\left\{\tau_{k}=\infty\right\}}\right\|_{L^{\varphi_{1 / r}[0,1)}} \leq C\left\|\sum_{k \in \mathbb{Z}} 2^{k r} \mathbf{1}_{\left\{\tau_{k}<\infty\right\}}\right\|_{L^{\varphi_{1 / r}[0,1)}},
$$

where $C$ is a positive constant and, for any $k \in \mathbb{Z}, \tau_{k}$ is the stopping time associated with the

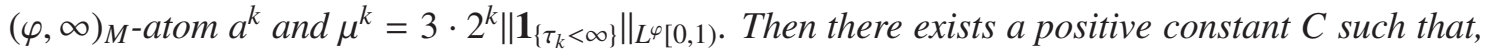
for any $f \in H_{\varphi}[0,1)$,

$$
\|T(f)\|_{L^{\varphi}[0,1)} \leq C\|f\|_{H_{\varphi}[0,1)} .
$$

Proof. Let $f \in H_{\varphi}[0,1)$. By Corollary 5.19 and Theorem 4.6, we know that there exist a sequence $\left(a^{k}\right)_{k \in \mathbb{Z}}$ of $(\varphi, \infty)_{M}$-atoms such that

$$
f=\sum_{k \in \mathbb{Z}} \mu^{k} a^{k} \quad \text { and } \quad\left\|\left[\sum_{k \in \mathbb{Z}}\left(3 \cdot 2^{k}\right)^{r} \mathbf{1}_{\left\{\tau_{k}<\infty\right\}}\right]^{1 / r}\right\|_{L^{\varphi}[0,1)} \lesssim\|f\|_{H_{\varphi}[0,1)},
$$

where the sequence $\left(\tau_{k}\right)_{k \in \mathbb{Z}}$ are stopping times, respectively, associated with $\left(a^{k}\right)_{k \in \mathbb{Z}}$ and, for any $k \in \mathbb{Z}, \mu^{k}:=3 \cdot 2^{k}\left\|\mathbf{1}_{\left\{\tau_{k}<\infty\right\}}\right\|_{L^{\varphi}[0,1)}$. Then we have

$$
\|T(f)\|_{L^{\varphi}[0,1)} \leq\left\|\sum_{k \in \mathbb{Z}} \mu^{k} T\left(a^{k}\right) \mathbf{1}_{\left\{\tau_{k}<\infty\right\}}\right\|_{L^{\varphi}[0,1)}+\left\|\sum_{k \in \mathbb{Z}} \mu^{k} T\left(a^{k}\right) \mathbf{1}_{\left\{\tau_{k}=\infty\right\}}\right\|_{L^{\varphi}[0,1)}=: \mathrm{B}_{1}+\mathrm{B}_{2} .
$$

We first estimate $\mathrm{B}_{1}$. Notice that, for any $k \in \mathbb{Z}$, the sets $\left\{\tau_{k}=\ell\right\}_{\ell \in \mathbb{Z}_{+}}$are disjoint and, for any $\ell \in \mathbb{Z}_{+}$, there exist a finite set $\Lambda_{k, \ell}$ and disjoint atoms $\left(I_{k, \ell, m}\right)_{m \in \Lambda_{k, \ell}} \subset \mathcal{F}_{\ell}$ such that $\left\{\tau_{k}=\ell\right\}=$ $\bigcup_{m \in \Lambda_{k, \ell}} I_{k, \ell, m}$. Thus, for any $k \in \mathbb{Z}$, we have

$$
\left\{\tau_{k}<\infty\right\}=\bigcup_{\ell \in \mathbb{Z}_{+}} \bigcup_{m \in \Lambda_{k, \ell}} I_{k, \ell, m}
$$

where $\left\{I_{k, \ell, m}\right\}_{\ell \in \mathbb{Z}_{+}, m \in \Lambda_{k, \ell}}$ are disjoint for fixed $k$. From this and $r \in(0,1]$, we deduce that

$$
\mathrm{B}_{1} \leq\left\|\sum_{k \in \mathbb{Z}}\left(\mu^{k}\right)^{r} \sum_{\ell \in \mathbb{Z}_{+}} \sum_{m \in \Lambda_{k, \ell}}\left|T\left(a^{k}\right)\right|^{r} \mathbf{1}_{I_{k, \ell, m}}\right\|_{L^{\varphi_{1 / r}[0,1)}}^{1 / r} .
$$

Since $r \leq p_{\varphi}^{-}$, by Lemma 2.13 and Remark 2.10, we know that $\varphi_{1 / r}$ is of uniformly lower type 1 . By this and Lemma 2.18, we can choose a function $g \in L^{\varphi_{1 / r}^{*}}[0,1)$ with norm less than or equal to 1 such that

$$
\left\|\sum_{k \in \mathbb{Z}}\left(\mu^{k}\right)^{r} \sum_{\ell \in \mathbb{Z}_{+}} \sum_{m \in \Lambda_{k, \ell}}\left|T\left(a^{k}\right)\right|^{r} \mathbf{1}_{I_{k, \ell, m}}\right\|_{L^{\varphi} / r[0,1)} \lesssim \int_{0}^{1} \sum_{k \in \mathbb{Z}}\left(\mu^{k}\right)^{r} \sum_{\ell \in \mathbb{Z}_{+}} \sum_{m \in \Lambda_{k, \ell}}\left|T\left(a^{k}\right)\right|^{r} \mathbf{1}_{I_{k, \ell, m}} g d \mathbb{P} .
$$

Combining this, the boundedness of $T$ on $L^{\infty}[0,1)$ and (7.6), we obtain

$$
\left(\mathrm{B}_{1}\right)^{r} \leq \int_{0}^{1} \sum_{k \in \mathbb{Z}}\left(\mu^{k}\right)^{r} \sum_{\ell \in \mathbb{Z}_{+}} \sum_{m \in \Lambda_{k, \ell}}\left|T\left(a^{k}\right)\right|^{r} \mathbf{1}_{I_{k, \ell}, m} g d \mathbb{P}
$$




$$
\begin{aligned}
& \lesssim \sum_{k \in \mathbb{Z}}\left(\mu^{k}\right)^{r} \sum_{\ell \in \mathbb{Z}_{+}} \sum_{m \in \Lambda_{k, \ell}}\left\|T\left(a^{k}\right)\right\|_{L^{\infty}[0,1)}^{r}\left\|\mathbf{1}_{I_{k, \ell, m}} g\right\|_{L^{1}(\Omega)} \\
& \lesssim \sum_{k \in \mathbb{Z}} \sum_{\ell \in \mathbb{Z}_{+}} \sum_{m \in \Lambda_{k, \ell}}\left(3 \cdot 2^{k}\right)^{r}\left\|\mathbf{1}_{\left\{\tau_{k}<\infty\right\}}\right\|_{L^{\varphi}[0,1)}^{r}\left\|a^{k}\right\|_{L^{\infty}[0,1)}^{r}\left\|\mathbf{1}_{I_{k, \ell, m}} g\right\|_{L^{1}(\Omega)} \\
& \lesssim \sum_{k \in \mathbb{Z}} \sum_{\ell \in \mathbb{Z}_{+}} \sum_{m \in \Lambda_{k, \ell}}\left(3 \cdot 2^{k}\right)^{r} \mathbb{P}\left(I_{k, \ell, m}\right)\left[\frac{1}{\mathbb{P}\left(I_{k, \ell, m}\right)} \int_{I_{k, \ell, m}}|g| d \mathbb{P}\right]
\end{aligned}
$$

which, together with the definition of the maximal operator and Lemma 2.18, further implies that

$$
\begin{aligned}
\left(\mathrm{B}_{1}\right)^{r} & \lesssim \sum_{k \in \mathbb{Z}} \sum_{\ell \in \mathbb{Z}_{+}} \sum_{m \in \Lambda_{k, \ell}}\left(3 \cdot 2^{k}\right)^{r} \int_{0}^{1} \mathbf{1}_{I_{k, \ell, m}} M(g) d \mathbb{P} \\
& \lesssim\left\|\sum_{k \in \mathbb{Z}} \sum_{\ell \in \mathbb{Z}_{+}} \sum_{m \in \Lambda_{k, \ell}}\left(3 \cdot 2^{k}\right)^{r} \mathbf{1}_{I_{k, \ell, m}}\right\|_{L^{\varphi / r}[0,1)}\|M(g)\|_{L^{\varphi_{1 / r}^{*}[0,1)}} .
\end{aligned}
$$

From this, the boundedness of $M$ on $L^{\varphi_{1 / r}^{*}}[0,1)$ and (7.4), it follows that

$$
\begin{aligned}
\mathrm{B}_{1} & \lesssim\left\|\sum_{k \in \mathbb{Z}} \sum_{\ell \in \mathbb{Z}_{+}} \sum_{m \in \Lambda_{k, \ell}}\left(3 \cdot 2^{k}\right)^{r} \mathbf{1}_{I_{k, \ell, m}}\right\|_{L^{\varphi_{1 / r}[0,1)}}^{1 / r}\|g\|_{L^{\varphi_{1 / r}^{*}[0,1)}}^{1 / r} \\
& \lesssim\left\|\sum_{k \in \mathbb{Z}}\left(3 \cdot 2^{k}\right)^{r} \mathbf{1}_{\left\{\tau_{k}<\infty\right\}}\right\|_{L^{\varphi_{1 / r}[0,1)}}^{1 / r} \sim\left\|\left[\sum_{k \in \mathbb{Z}}\left(3 \cdot 2^{k}\right)^{r} \mathbf{1}_{\left\{\tau_{k}<\infty\right\}}\right]^{1 / r}\right\|_{L^{\varphi}[0,1)} \lesssim\|f\|_{H_{\varphi}[0,1)} .
\end{aligned}
$$

On another hand, by (7.3) and (7.4), we have

$$
\mathrm{B}_{2} \leq\left\|\sum_{k \in \mathbb{Z}}\left(\mu^{k}\right)^{r}\left|T\left(a^{k}\right)\right|^{r} \mathbf{1}_{\left\{\tau_{k}=\infty\right\}}\right\|_{L^{\varphi_{1 / r}[0,1)}}^{1 / r} \lesssim\left\|\sum_{k \in \mathbb{Z}} 2^{k r} \mathbf{1}_{\left\{\tau_{k}<\infty\right\}}\right\|_{L^{\varphi_{1 / r}[0,1)}}^{1 / r} \lesssim\|f\|_{H_{\varphi}[0,1)},
$$

which completes the proof Theorem 7.4.

Theorem 7.5. Let $\varphi \in A_{\infty}[0,1)$ be a Musielak-Orlicz function with uniformly lower type $p_{\varphi}^{-}$and uniformly upper type $p_{\varphi}^{+}$. Suppose that the Doob maximal operator $M$ is bounded on $L^{\varphi_{1 / r}^{*}}[0,1)$ for some $r \in\left(\frac{1}{2}, 1 \wedge p_{\varphi}^{-}\right]$. Then there exists a positive constant $C$ such that

$$
\left\|\sum_{k \in \mathbb{Z}}\left(\mu^{k}\right)^{r}\left[\sigma_{*}\left(a^{k}\right)\right]^{r} \mathbf{1}_{\left\{\tau_{k}=\infty\right\}}\right\|_{L^{\varphi_{1 / r}[0,1)}} \leq C\left\|\sum_{k \in \mathbb{Z}} 2^{k r} \mathbf{1}_{\left\{\tau_{k}<\infty\right\}}\right\|_{L^{\varphi_{1 / r}[0,1)}},
$$

where, for any $k \in \mathbb{Z}, \tau_{k}$ is the stopping time associated with the $(\varphi, \infty)_{M}$-atom $a^{k}$ and $\mu^{k}:=$ $3 \cdot 2^{k}\left\|\mathbf{1}_{\left\{\tau_{k}<\infty\right\}}\right\|_{L^{\varphi}[0,1)}$.

Proof. For any $k \in \mathbb{Z}$ and $\ell \in \mathbb{Z}_{+}$, let $\Lambda_{k, \ell}$ be as in (7.5). Moreover, for any $m \in \Lambda_{k, \ell}$, let $I_{k, \ell, m}$ be also as in (7.5) and $K_{k, \ell, m} \in \mathbb{N}$ satisfying $\mathbb{P}\left(I_{k, \ell, m}\right)=2^{-K_{k, \ell, m}}$. It was proved in [40, Theorem 7.14] that, for any $k \in \mathbb{Z}$ and $x \in\left\{\tau_{k}=\infty\right\}$,

$$
\sigma_{*}\left(a^{k}\right)(x) \lesssim\left\|\mathbf{1}_{\left\{\tau_{k}<\infty\right\}}\right\|_{L^{\varphi}[0,1)}^{-1}\left[\sum_{\ell \in \mathbb{Z}_{+}} \sum_{m \in \Lambda_{k, \ell}} \sum_{j=0}^{K_{k, \ell, m}-1} 2^{j-K_{k, \ell, m}} \mathbf{1}_{I_{k, \ell, m}+2^{-j-1}}(x)\right.
$$




$$
\begin{aligned}
& \left.+\sum_{\ell \in \mathbb{Z}_{+}} \sum_{m \in \Lambda_{k, \ell}} \sum_{j=0}^{K_{k, \ell, m}-1} 2^{j-K_{k, \ell, m}} \sum_{i=j}^{K_{k, \ell, m}-1} 2^{i-K_{k, \ell, m}} \mathbf{1}_{I_{k, \ell, m}+\left[2^{-j-1}, 2^{-j-1}+2^{-i}\right)}(x)\right] \\
=: & \left\|\mathbf{1}_{\left\{\tau_{k}<\infty\right\}}\right\|_{L^{\varphi}[0,1)}^{-1}\left[\mathrm{~A}_{k}(x)+\mathrm{B}_{k}(x)\right],
\end{aligned}
$$

which implies that

$$
\begin{gathered}
\left\|\sum_{k \in \mathbb{Z}}\left(\mu^{k}\right)^{r}\left[\sigma_{*}\left(a^{k}\right)\right]^{r} \mathbf{1}_{\left\{\tau_{k}=\infty\right\}}\right\|_{L^{\varphi_{1 / r}[0,1)}} \\
=: \sum_{k \in \mathbb{Z}} 2^{k r}\left(\mathrm{~A}_{k}\right)^{r}\left\|_{L^{\varphi_{1 / r}[0,1)}}+\right\| \sum_{k \in \mathbb{Z}} 2^{k r}\left(\mathrm{~B}_{k}\right)^{r} \|_{L^{\varphi_{1 / r}[0,1)}}
\end{gathered}
$$

By Lemma 2.18, there exists a function $g \in L^{\varphi_{1 / r}^{*}}[0,1)$ with norm less than or equal to 1 such that

$$
\begin{aligned}
Z_{1} & \lesssim \int_{0}^{1} \sum_{k \in \mathbb{Z}} 2^{k r}\left(A_{k}\right)^{r} g d \mathbb{P} \sim \int_{0}^{1} \sum_{k \in \mathbb{Z}} 2^{k r} \sum_{\ell \in \mathbb{Z}_{+}} \sum_{m \in \Lambda_{k, \ell}} \sum_{j=0}^{K_{k, \ell, m}-1} 2^{\left(j-K_{k, \ell, m}\right) r} \mathbf{1}_{I_{k, \ell, m}+2^{-j-1}} g d \mathbb{P} \\
& \lesssim \sum_{k \in \mathbb{Z}} 2^{k r} \sum_{\ell \in \mathbb{Z}_{+}} \sum_{m \in \Lambda_{k, \ell}} \sum_{j=0}^{K_{k, l, m}-1} 2^{\left(j-K_{k, l, m}\right) r}\left|\int_{I_{k, \ell, m}+2^{-j-1}} g d \mathbb{P}\right| \\
& \lesssim \sum_{k \in \mathbb{Z}} 2^{k r} \sum_{\ell \in \mathbb{Z}_{+}} \sum_{m \in \Lambda_{k, \ell}} \sum_{j=0}^{K_{k, \ell, m}-1} 2^{\left(j-K_{k, \ell, m}\right) r} \int_{0}^{1} \mathbf{1}_{I_{k, \ell, m}} \frac{1}{\mathbb{P}\left(I_{k, \ell, m} \dot{+} 2^{-j-1}\right)}\left|\int_{I_{k, \ell, m}+2^{-j-1}} g d \mathbb{P}\right| d \mathbb{P},
\end{aligned}
$$

where the last inequality follows from $\mathbb{P}\left(I_{k, \ell, m}\right)=\mathbb{P}\left(I_{k, \ell, m} \dot{+} 2^{-j-1}\right)=2^{-K_{k, \ell, m}}$. From this and Lemma 2.18 , we deduce that

$$
\begin{aligned}
\mathrm{Z}_{1} & \lesssim \int_{0}^{1} \sum_{k \in \mathbb{Z}} 2^{k r} \sum_{\ell \in \mathbb{Z}_{+}} \sum_{m \in \Lambda_{k, \ell}} \mathbf{1}_{I_{k, \ell, m}} \sum_{j=0}^{K_{k, \ell, m}-1} 2^{\left(j-K_{k, \ell, m}\right) r} \frac{1}{\mathbb{P}\left(I_{k, \ell, m} \dot{+} 2^{-j-1}\right)}\left|\int_{I_{k, \ell, m}+2^{-j-1}} g d \mathbb{P}\right| d \mathbb{P} \\
& \lesssim \int_{0}^{1} \sum_{k \in \mathbb{Z}} 2^{k r} \sum_{\ell \in \mathbb{Z}_{+}} \sum_{m \in \Lambda_{k, \ell}} \mathbf{1}_{I_{k, \ell, m}} U_{r, K_{k, \ell, m}}(g) d \mathbb{P} \\
& \lesssim\left\|\sum_{k \in \mathbb{Z}} 2^{k r} \sum_{\ell \in \mathbb{Z}_{+}} \sum_{m \in \Lambda_{k, \ell}} \mathbf{1}_{I_{k, \ell, m}}\right\|_{L^{\varphi_{1 / r}[0,1)}}\left\|U_{r, K_{k, \ell, m}}(g)\right\|_{L^{\varphi_{1 / r}^{*}[0,1)}} .
\end{aligned}
$$

By this, $\|g\|_{L^{\varphi_{1 / r}^{*}}[0,1)} \leq 1$ and Theorem 7.3, we conclude that

$$
\mathrm{Z}_{1} \lesssim\left\|\sum_{k \in \mathbb{Z}} \sum_{\ell \in \mathbb{Z}_{+}} \sum_{m \in \Lambda_{k, \ell}} 2^{k r} \mathbf{1}_{I_{k, \ell, m}}\right\|_{L^{\varphi_{1 / r}[0,1)}}\|g\|_{L^{\varphi_{1 / r}^{*}[0,1)}} \lesssim\left\|\sum_{k \in \mathbb{Z}} 2^{k r} \mathbf{1}_{\left\{\tau_{k}<\infty\right\}}\right\|_{L^{\varphi_{1 / r}[0,1)}} .
$$

For $\mathrm{Z}_{2}$, we choose again a function $g \in L^{\varphi_{1 / r}^{*}}[0,1)$ with $\|g\|_{L^{\varphi_{1 / r}^{*}[0,1)}} \leq 1$ such that

$$
\mathrm{Z}_{2} \lesssim \int_{0}^{1} \sum_{k \in \mathbb{Z}} 2^{k r}\left(B_{k}\right)^{r} g d \mathbb{P} \text {. }
$$


It is clear that, for any $k \in \mathbb{Z}, i, j, \ell \in \mathbb{Z}_{+}, m \in \Lambda_{k, \ell}$ and $j \leq i \leq K_{k, \ell, m}-1$,

$$
\mathbb{P}\left(I_{k, \ell, m} \dot{+}\left[2^{-j-1}, 2^{-j-1} \dot{+} 2^{-i}\right)\right)=2^{-i} .
$$

From this and (7.11), it follows that

$$
\begin{aligned}
\mathrm{Z}_{2} & \lesssim \int_{0}^{1} \sum_{k \in \mathbb{Z}} 2^{k r} \sum_{\ell \in \mathbb{Z}_{+}} \sum_{m \in \Lambda_{k, \ell}} \sum_{j=0}^{K_{k, \ell, m}-1} \sum_{i=j}^{K_{k, \ell, m}-1} 2^{\left(j-K_{k, \ell, m}\right) r} 2^{\left(i-K_{k, \ell, m}\right) r} \mathbf{1}_{I_{k, \ell, m}+\left[2^{-j-1}, 2^{-j-1}+2^{-i}\right)} g d \mathbb{P} \\
\lesssim & \sum_{k \in \mathbb{Z}} 2^{k r} \sum_{\ell \in \mathbb{Z}_{+}} \sum_{m \in \Lambda_{k, \ell}} \sum_{j=0}^{K_{k, \ell, m}-1} \sum_{i=j}^{K_{k, \ell, m}-1} 2^{\left(j-K_{k, \ell, m}\right) r} 2^{\left(i-K_{k, \ell, m}\right) r}\left|\int_{I_{k, \ell, m}+\left[2^{-j-1}, 2^{-j-1}+2^{-i}\right)} g d \mathbb{P}\right| \\
\lesssim & \int_{0}^{1} \sum_{k \in \mathbb{Z}} 2^{k r} \sum_{\ell \in \mathbb{Z}_{+}} \sum_{m \in \Lambda_{k, \ell}} \sum_{j=0}^{K_{k, \ell, m}-1} \sum_{i=j}^{K_{k, \ell, m}-1} 2^{\left(j-K_{k, \ell, m}\right) r} 2^{\left(i-K_{k, \ell, m}\right) r} 2^{K_{k, \ell, m}-i} \mathbf{1}_{I_{k, \ell, m}} \\
& \times \frac{1}{\mathbb{P}\left(I_{k, \ell, m} \dot{+}\left[2^{-j-1}, 2^{-j-1} \dot{+} 2^{-i}\right)\right)}\left|\int_{I_{k, \ell, m} \dot{+}\left[2^{-j-1}, 2^{-j-1}+2^{-i}\right)} g d \mathbb{P}\right| d \mathbb{P},
\end{aligned}
$$

which implies that

$$
\mathrm{Z}_{2} \lesssim \int_{0}^{1} \sum_{k \in \mathbb{Z}} 2^{k r} \sum_{\ell \in \mathbb{Z}_{+}} \sum_{m \in \Lambda_{k, \ell}} \mathbf{1}_{I_{k, \ell, m}} V_{r, K_{k, \ell, m}}(g) d \mathbb{P} .
$$

Combining this, Lemma 2.18 and Theorem 7.3, we conclude that

$$
\begin{aligned}
\mathrm{Z}_{2} & \lesssim\left\|\sum_{k \in \mathbb{Z}} 2^{k r} \sum_{\ell \in \mathbb{Z}_{+}} \sum_{m \in \Lambda_{k, \ell}} \mathbf{1}_{I_{k, \ell, m}}\right\|_{L^{\varphi_{1 / r}[0,1)}}\left\|V_{r, K_{k, \ell, m}}(g)\right\|_{L^{\varphi_{1 / r}^{*}[0,1)}} \\
& \lesssim\left\|\sum_{k \in \mathbb{Z}} \sum_{\ell \in \mathbb{Z}_{+}} \sum_{m \in \Lambda_{k, \ell}} 2^{k r} \mathbf{1}_{I_{k, \ell, m}}\right\|_{L^{\varphi_{1 / r}[0,1)}}\|g\|_{L^{\varphi_{1 / r}^{*}[0,1)}} \lesssim\left\|\sum_{k \in \mathbb{Z}} 2^{k r} \mathbf{1}_{\left\{\tau_{k}<\infty\right\}}\right\|_{L^{\varphi_{1 / r}[0,1)}} .
\end{aligned}
$$

From this, (7.9) and (7.10), we deduce the desired conclusion. This finishes the proof of Theorem 7.5 .

Remark 7.6. Notice that, under the assumptions of Theorem 7.5, we have $p_{\varphi}^{-} \in\left(\frac{1}{2}, \infty\right)$.

Theorem 7.7. Let $\varphi \in A_{\infty}[0,1)$ be a Musielak-Orlicz function with uniformly lower type $p_{\varphi}^{-}$and uniformly upper type $p_{\varphi}^{+}$. Suppose that the Doob maximal operator $M$ is bounded on $L^{\varphi_{1 / r}^{*}}[0,1)$ for some $r \in\left(\frac{1}{2}, \min \left\{1, p_{\varphi}^{-}\right\}\right]$. Then there exists a positive constant $C$ such that, for any $f \in H_{\varphi}[0,1)$,

$$
\left\|\sigma_{*} f\right\|_{L^{\varphi}[0,1)} \leq C\|f\|_{H_{\varphi}[0,1)}
$$

Proof. The conclusions of this theorem follows from Theorems 7.4 and 7.5 immediately. 
Remark 7.8. If the condition, that the Doob maximal operator is bounded on $L^{\varphi_{1 / r}^{*}}[0,1)$ for some $r \in\left(\frac{1}{2}, \min \left\{1, p_{\varphi}^{-}\right\}\right]$in Theorem 7.7, is replaced by the condition that $\varphi_{1 / r}^{*} \in A_{\infty}[0,1)$ and

$$
q\left(\varphi_{1 / r}^{*}\right)<\left(p_{\varphi}^{+} / r\right)^{\prime} \leq\left(p_{\varphi}^{-} / r\right)^{\prime}<\infty
$$

for some $r \in\left(\frac{1}{2}, \min \left\{1, p_{\varphi}^{-}\right\}\right]$, then, from Corollary 3.4, we deduce that the conclusion of Theorem 7.7 still holds true.

Notice that Theorem 7.7 is new even for the weighted Hardy spaces [namely, for any given $p \in\left(\frac{1}{2}, \infty\right), \varphi(x, t):=w(x) t^{p}$ for any $x \in[0,1)$ and $\left.t \in(0, \infty)\right]$ and for Orlicz Hardy spaces [namely, $\varphi(x, t)=\Phi(t)$ for any $x \in[0,1)$ and $t \in(0, \infty)$, here $\Phi$ is an Orlicz function].

Theorem 7.9. Let $p \in\left(\frac{1}{2}, \infty\right)$ and $w \in A_{2 p}[0,1)$ be a special weight. Let $\varphi(x, t):=w(x) t^{p}$ for any $x \in[0,1)$ and $t \in(0, \infty)$. Then there exists a positive constant $C$ such that, for any $f \in H_{\varphi}[0,1)$,

$$
\left\|\sigma_{*} f\right\|_{L^{\varphi}[0,1)} \leq C\|f\|_{H_{\varphi}[0,1)} .
$$

Proof. Clearly, $\varphi$ is of uniformly lower type $p$ and of uniformly upper type $p$. It is well known that $w \in A_{2 p}[0,1)$ implies that there exists $r \in\left(\frac{1}{2}, \min \{1, p\}\right)$ such that $w \in A_{p / r}[0,1)$. By Example 2.16(i), we know that, for any $x \in[0,1)$ and $t \in(0, \infty)$,

$$
\varphi_{1 / r}^{*}(x, t)=[w(x)]^{\frac{-1}{p / r-1}} t^{(p / r)^{\prime}}(p / r)^{\frac{-1}{p / r-1}} \frac{1}{(p / r)^{\prime}} .
$$

Observe that $\varphi_{1 / r}^{*}$ is of uniformly lower type $(p / r)^{\prime}$ and of uniformly upper type $(p / r)^{\prime}$. We can easily check that $w \in A_{p / r}[0,1)$ implies $w^{\frac{-1}{p / r-1}} \in A_{(p / r)^{\prime}}[0,1)$. From this, it follows that $\varphi_{1 / r}^{*} \in$ $A_{(p / r)^{\prime}}[0,1)$. Then there exists $\varepsilon \in(0, \infty)$ such that $\varphi_{1 / r}^{*} \in A_{(p / r)^{\prime}-\varepsilon}[0,1)$. Thus, we have $q\left(\varphi_{1 / r}^{*}\right)<$ $(p / r)^{\prime}$ and hence $\varphi_{1 / r}^{*}$ satisfies (7.12). The desired conclusion of the theorem follows from Remark 7.8. This finishes the proof of Theorem 7.9.

Theorem 7.7 gives back the well known result of Weisz ([74, 78]) mentioned above when $\varphi(x, t):=t^{p}$ for any $x \in[0,1)$ and $t \in(0, \infty)$, with $p \in\left(\frac{1}{2}, \infty\right)$. For $p=1$, it is due to Fujii [22] (see also Schipp et al. [67]). If $\varphi(x, t):=t^{p}$ for any $x \in[0,1)$ and $t \in(0, \infty)$, with $p \in\left(0, \frac{1}{2}\right]$, then Theorem 7.7 is not true anymore (see Simon et al. [70], Simon [69] and Gát et al. [24]).

Theorem 7.10. Let $\Phi$ be an Orlicz function with lower type $p_{\Phi}^{-}$and upper type $p_{\Phi}^{+}$and $\varphi(x, t):=$ $\Phi(t)$ for any $x \in[0,1)$ and $t \in(0, \infty)$. If $p_{\Phi}^{-} \in(1 / 2, \infty)$, then there exists a positive constant $C$ such that, for any $f \in H_{\varphi}[0,1)$,

$$
\left\|\sigma_{*} f\right\|_{L^{\varphi}[0,1)} \leq C\|f\|_{H_{\varphi}[0,1)} .
$$

Proof. Choosing $r \in\left(\frac{1}{2}, \min \left\{1, p_{\Phi}^{-}\right\}\right)$, we can apply Remark 7.8. Then the desired conclusion of this theorem follows from Lemmas 2.13 and 2.15 and the fact that $q\left(\varphi_{1 / r}^{*}\right)=1$. This finishes the proof of Theorem 7.10.

By standard arguments (see, for example, Jiao et al. [40]), Theorem 7.7 implies the next convergence results of $\left(\sigma_{n} f\right)_{n \in \mathbb{N}}$. We state these convergence results only in the general case, more exactly, under the assumptions of Theorem 7.7. Let $p \in\left(\frac{1}{2}, \infty\right), w \in A_{2 p}[0,1)$ be a special weight and $\Phi$ an Orlicz function. Obviously, we could formulate the convergence results if $\varphi(x, t):=w(x) t^{p}$ or if $\varphi(x, t):=\Phi(t)$ for any $x \in[0,1)$ and $t \in(0, \infty)$, in other words, under the assumptions of Theorems 7.9 or 7.10 , which are new results as well. 
Corollary 7.11. Let $\varphi \in A_{\infty}[0,1)$ be a Musielak-Orlicz function with uniformly lower type $p_{\varphi}^{-}$and uniformly upper type $p_{\varphi}^{+}$. Suppose that the Doob maximal operator $M$ is bounded on $L^{\varphi_{1 / r}^{*}}[0,1)$ for some $r \in\left(\frac{1}{2}, \min \left\{1, p_{\varphi}^{-}\right\}\right]$. If $f \in H_{\varphi}[0,1)$, then $\sigma_{n} f$ converges almost everywhere on $[0,1)$ as well as in the $L^{\varphi}[0,1)$-norm as $n \rightarrow \infty$.

For any integrable function $f$, the limit of $\left(\sigma_{n} f\right)_{n \in \mathbb{N}}$ is exactly the function. For any $k \in \mathbb{Z}_{+}$, let $I \in \mathbb{F}_{k}$ be an atom of $\mathbb{F}_{k}$. The restriction of a martingale $f$ to the atom $I$ is defined by setting, for any $n \in \mathbb{Z}_{+}$,

$$
f \mathbf{1}_{I}:=\left(\mathbb{E}_{n} f \mathbf{1}_{I}, n \geq k\right) .
$$

Corollary 7.12. Let $\varphi \in A_{\infty}[0,1)$ be a Musielak-Orlicz function with uniformly lower type $p_{\varphi}^{-}$and uniformly upper type $p_{\varphi}^{+}$. Suppose that the Doob maximal operator $M$ is bounded on $L^{\varphi_{1 / r}^{*}}[0,1)$ for some $r \in\left(\frac{1}{2}, \min \left\{1, p_{\varphi}^{-}\right\}\right]$. If $f \in H_{\varphi}[0,1)$ and there exists a dyadic interval $I$ such that the restriction $f \mathbf{1}_{I} \in L^{1}(I)$, then

$$
\lim _{n \rightarrow \infty} \sigma_{n} f(x)=f(x) \quad \text { for almost every } x \in I \text { as well as in the } L^{\varphi}(I) \text {-norm. }
$$

Corollary 7.13. Let $\varphi \in A_{\infty}[0,1)$ be a Musielak-Orlicz function with uniformly lower type $p_{\varphi}^{-}$and uniformly upper type $p_{\varphi}^{+}$satisfying (3.2). Suppose that the Doob maximal operator $M$ is bounded on $L^{\varphi_{1 / r}^{*}}[0,1)$ for some $r \in\left(\frac{1}{2}, \min \left\{1, p_{\varphi}^{-}\right\}\right]$. If $f \in L^{\varphi}[0,1)$, then

$$
\lim _{n \rightarrow \infty} \sigma_{n} f(x)=f(x) \quad \text { for almost every } x \in[0,1) \text { as well as in the } L^{\varphi}[0,1) \text {-norm. }
$$

Proof. By Theorem 3.2 and $f \in L^{\varphi}[0,1)$, we know that $f \in H_{\varphi}[0,1)$ and hence $f$ is integrable. The desired conclusion follows from Corollary 7.12. This finishes the proof of Corollary 7.13.

Considering only $\left(\sigma_{2^{n}}\right)_{n \in \mathbb{N}}$, we do not need the restriction $r \in\left(\frac{1}{2}, 1\right]$.

Theorem 7.14. Let $\varphi \in A_{\infty}[0,1)$ be a Musielak-Orlicz function with uniformly lower type $p_{\varphi}^{-}$and uniformly upper type $p_{\varphi}^{+}$. Suppose that the Doob maximal operator $M$ is bounded on $L^{\varphi_{1 / r}^{*}}[0,1)$ for some $r \in\left(0, \min \left\{1, p_{\varphi}^{-}\right\}\right]$. Then there exists a positive constant $C$ such that

$$
\left\|\sum_{k \in \mathbb{Z}}\left(\mu^{k}\right)^{r} \sup _{n \in \mathbb{N}}\left|\sigma_{2^{n}}\left(a^{k}\right)\right|^{r} \mathbf{1}_{\left\{\tau_{k}=\infty\right\}}\right\|_{L^{\varphi_{1 / r}[0,1)}} \leq C\left\|\sum_{k \in \mathbb{Z}} 2^{k r} \mathbf{1}_{\left\{\tau_{k}<\infty\right\}}\right\|_{L^{\varphi_{1 / r}[0,1)}},
$$

where, for any $k \in \mathbb{Z}, \tau_{k}$ is the stopping time associated with the $(\varphi, \infty)_{M}$-atom $a^{k}$ and $\mu^{k}:=$ $3 \cdot 2^{k}\left\|\mathbf{1}_{\left\{\tau_{k}<\infty\right\}}\right\|_{L^{\varphi}[0,1)}$.

Proof. Similarly to (7.8), for any $k \in \mathbb{Z}$ and $\ell \in \mathbb{Z}_{+}$, let $\Lambda_{k, \ell}$ be defined as in (7.5). Moreover, for any $m \in \Lambda_{k, \ell}$, let $I_{k, \ell, m}$ be defined also as in (7.5) and $K_{k, \ell, m} \in \mathbb{N}$ satisfying $\mathbb{P}\left(I_{k, \ell, m}\right)=2^{-K_{k, \ell, m}}$. It was proved in [40, Theorem 7.14] that, for any $k \in \mathbb{Z}$ and $x \in\left\{\tau_{k}=\infty\right\}$,

$$
\sup _{n \in \mathbb{N}}\left|\sigma_{2^{n}}\left(a^{k}\right)(x)\right| \lesssim\left\|\mathbf{1}_{\{\tau<\infty\}}\right\|_{L^{\varphi}[0,1)}^{-1} \sum_{\ell \in \mathbb{Z}_{+}} \sum_{m \in \Lambda_{k, \ell}} \sum_{j=0}^{K_{k, \ell, m}-1} 2^{j-K_{k, \ell, m}} \mathbf{1}_{I_{k, \ell, m}+2^{-j-1}}(x) .
$$

Then the proof of this theorem can be finished as in Theorem 7.5. 
We deduce the next result from this and Theorem 7.4.

Theorem 7.15. Let $\varphi \in A_{\infty}[0,1)$ be a Musielak-Orlicz function with uniformly lower type $p_{\varphi}^{-}$and uniformly upper type $p_{\varphi}^{+}$. Suppose that the Doob maximal operator $M$ is bounded on $L^{\varphi_{1 / r}^{*}}[0,1)$ for some $r \in\left(0, \min \left\{1, p_{\varphi}^{-}\right\}\right]$. Then there exists a positive constant $C$ such that, for any $f \in H_{\varphi}[0,1)$,

$$
\left\|\sup _{n \in \mathbb{N}}\left|\sigma_{2^{n}} f\right|\right\|_{L^{\varphi}[0,1)} \leq C\|f\|_{H_{\varphi}[0,1)} .
$$

The following corollaries can be proved as Corollaries 7.11, 7.12 and 7.13.

Corollary 7.16. Let $\varphi \in A_{\infty}[0,1)$ be a Musielak-Orlicz function with uniformly lower type $p_{\varphi}^{-}$and uniformly upper type $p_{\varphi}^{+}$. Suppose that the Doob maximal operator $M$ is bounded on $L^{\varphi_{1 / r}^{*}}[0,1)$ for some $r \in\left(0, \min \left\{1, p_{\varphi}^{-}\right\}\right]$. If $f \in H_{\varphi}[0,1)$, then $\sigma_{2^{n}} f$ converges almost everywhere on $[0,1)$ as well as in the $L^{\varphi}[0,1)$-norm as $n \rightarrow \infty$.

Corollary 7.17. Let $\varphi \in A_{\infty}[0,1)$ be a Musielak-Orlicz function with uniformly lower type $p_{\varphi}^{-}$and uniformly upper type $p_{\varphi}^{+}$. Suppose that the Doob maximal operator $M$ is bounded on $L^{\varphi_{1 / r}^{*}}[0,1)$ for some $r \in\left(0, \min \left\{1, p_{\varphi}^{-}\right\}\right]$. If $f \in H_{\varphi}[0,1)$ and there exists a dyadic interval I such that the restriction $f \mathbf{1}_{I} \in L^{1}(I)$, then

$$
\lim _{n \rightarrow \infty} \sigma_{2^{n}} f(x)=f(x) \quad \text { for almost every } x \in I \text { as well as in the } L^{\varphi}(I) \text {-norm. }
$$

Corollary 7.18. Let $\varphi \in A_{\infty}[0,1)$ be a Musielak-Orlicz function with uniformly lower type $p_{\varphi}^{-}$and uniformly upper type $p_{\varphi}^{+}$satisfying (3.2). Suppose that the Doob maximal operator $M$ is bounded on $L^{\varphi_{1 / r}^{*}}[0,1)$ for some $r \in\left(0, \min \left\{1, p_{\varphi}^{-}\right\}\right]$. If $f \in L^{\varphi}[0,1)$, then

$$
\lim _{n \rightarrow \infty} \sigma_{2^{n}} f(x)=f(x) \quad \text { for almost every } x \in[0,1) \text { as well as in the } L^{\varphi}[0,1) \text {-norm. }
$$

\section{References}

[1] K. F. Andersen and R. T. John, Weighted inequalities for vector-valued maximal functions and singular integrals, Studia Math. 69 (1980/81), 19-31.

[2] M. Avci and A. Pankov, Multivalued elliptic operators with nonstandard growth, Adv. Nonlinear Anal. 7 (2018), 35-48.

[3] R. Bañuelos and A. Osȩkowski, A weighted maximal inequality for differentially subordinate martingales, Proc. Amer. Math. Soc. 146 (2018), 2263-2275.

[4] R. Bañuelos and A. Osȩkowski, Weighted $L^{2}$ inequalities for square functions, Trans. Amer. Math. Soc. 370 (2018), 2391-2422.

[5] P. Baroni, M. Colombo and G. Mingione, Regularity for general functionals with double phase, Calc. Var. Partial Differential Equations 57 (2018), no. 2, Art. 62, 48 pp.

[6] A. Bonami, S. Grellier and L. D. Ky, Paraproducts and products of functions in $B M O\left(\mathbb{R}^{n}\right)$ and $\mathcal{H}^{1}\left(\mathbb{R}^{n}\right)$ through wavelets, J. Math. Pures Appl. (9) 97 (2012), 230-241.

[7] A. Bonami and D. Lépingle, Fonction maximale et variation quadratique des martingales en présence d'un poids, in: (French) Séminaire de Probabilités, XIII (Univ. Strasbourg, Strasbourg, 1977/78), pp. 294-306, Lecture Notes in Math. 721, Springer, Berlin, 1979. 
[8] A. Bonami, T. Iwaniec, P. Jones and M. Zinsmeister, On the product of functions in BMO and $H^{1}$, Ann. Inst. Fourier (Grenoble) 57 (2007), 1405-1439.

[9] D. L. Burkholder, Distribution function inequalities for martingales, Ann. Probability 1 (1973), 19-42.

[10] D. L. Burkholder, B. J. Davis and R. F. Gundy, Integral inequalities for convex functions of operators on martingales, in: Proceedings of the Sixth Berkeley Symposium on Mathematical Statistics and Probability (Univ. California, Berkeley, Calif., 1970/1971), Vol. II: Probability theory, pp. 223-240, Univ. California Press, Berkeley, Calif., 1972.

[11] D. L. Burkholder and R. F. Gundy, Extrapolation and interpolation of quasi-linear operators on martingales, Acta Math. 124 (1970), 249-304.

[12] J. Cao, D.-C. Chang, D. Yang and S. Yang, Riesz transform characterizations of MusielakOrlicz-Hardy spaces, Trans. Amer. Math. Soc. 368 (2016), 6979-7018.

[13] M. Colombo and G. Mingione, Bounded minimisers of double phase variational integrals, Arch. Ration. Mech. Anal. 218 (2015), 219-273.

[14] D. Cruz-Uribe and A. Fiorenza, Variable Lebesgue Spaces, Foundations and Harmonic analysis, Applied and Numerical Harmonic Analysis, Springer, Heidelberg, 2013.

[15] D. Cruz-Uribe and P. Hästö, Extrapolation and interpolation in genaralized Orlicz spaces, Trans. Amer. Math. Soc. 370 (2018), 4323-4349.

[16] D. Cruz-Uribe, J. M. Martell and C. Pérez, Weights, Extrapolation and the Theory of Rubio de Francia, Operator Theory: Advances and Applications, 215, Birkhäuser/Springer Basel AG, Basel, 2011.

[17] C. Doléans-Dade and P.-A. Meyer, Inégalités de normes avec poids, in: (French) Séminaire de Probabilités, XIII, (Univ. Strasbourg, Strasbourg, 1977/78), pp. 313-331, Lecture Notes in Math. 721, Springer, Berlin, 1979.

[18] S. J. Dilworth, Some probabilistic inequalities with applications to functional analysis, in: Banach spaces (Mérida, 1992), 53-67, Contemp. Math. 144, Amer. Math. Soc., Providence, RI, 1993.

[19] J. L. Doob, Stochastic Processes, John Wiley \& Sons, Inc., New York; Chapman \& Hall, Limited, London, 1953.

[20] C. Fefferman and E. M. Stein, Some maximal inequalities, Amer. J. Math. 93 (1971), 107115.

[21] N. J. Fine, On the Walsh functions, Trans. Amer. Math. Soc. 65 (1949), 372-414.

[22] N. Fujii, A maximal inequality for $H^{1}$-functions on a generalized Walsh-Paley group, Proc. Amer. Math. Soc. 77 (1979), 111-116.

[23] A. M. Garsia, Martingale Inequalities: Seminar Notes on Recent Progress, Mathematics Lecture Notes Series, W. A. Benjamin, Inc, Reading, Mass.-London-Amsterdam, 1973.

[24] G. Gát and U. Goginava, The weak type inequality for the maximal operator of the $(C, \alpha)$ means of the Fourier series with respect to the Walsh-Kaczmarz system, Acta Math. Hungar. 125 (2009), 65-83.

[25] B. Golubov, A. Efimov and V. Skvortsov, Walsh Series and Transforms, Kluwer Academic Publishers, Dordrecht, 1991.

[26] P. Gwiazda, I. Skrzypczak and A. Zatorska-Goldstein, Existence of renormalized solutions to elliptic equation in Musielak-Orlicz space, J. Differential Equations 264 (2018), 341-377. 
[27] P. Harjulehto, P. Hästö, V. Latvala and O. Toivanen, Critical variable exponent functionals in image restoration, Appl. Math. Lett. 26 (2013), 56-60.

[28] P. Harjulehto, P. Hästö and R. Klèn, Generalized Orlicz spaces and related PDE, Nonlinear Anal. 143 (2016), 155-173.

[29] K.-P. Ho, Atomic decomposition, dual spaces and interpolations of martingale LorentzKaramata spaces, Q. J. Math. 65 (2014), 985-1009.

[30] K.-P. Ho, Martingale inequalities on rearrangement-invariant quasi-Banach function spaces, Acta Sci. Math. (Szeged) 83 (2017), 619-627.

[31] K.-P. Ho, Doob's inequality, Burkholder-Gundy inequality and martingale transforms on martingale Morrey spaces, Acta Math. Sci. Ser. B (Engl. Ed.) 38 (2018), 93-109.

[32] T. Hytönen, J. van Neerven, M. Veraar and L. Weis, Analysis in Banach Spaces. Vol. I. Martingales and Littlewood-Paley Theory, Results in Mathematics and Related Areas, 3rd Series, A Series of Modern Surveys in Mathematics 63, Springer, Cham, 2016.

[33] M. Izumisawa and N. Kazamaki, Weighted norm inequalities for martingales, Tôhoku Math. J. (2) 29 (1977), 115-124.

[34] R. Jiang and D. Yang, New Orlicz-Hardy spaces associated with divergence form elliptic operators, J. Funct. Anal. 258 (2010), 1167-1224.

[35] Y. Jiao, L. Wu, A. Yang and R. Yi, The predual and John-Nirenberg inequalities on generalized BMO martingale spaces, Trans. Amer. Math. Soc. 369 (2017), 537-553.

[36] Y. Jiao, G. Xie and D. Zhou, Dual spaces and John-Nirenberg inequalities of martingale Hardy-Lorentz-Karamata spaces, Q. J. Math. 66 (2015), 605-623.

[37] Y. Jiao, L. Wu and M. Popa, Operator-valued martingale transforms in rearrangement invariant spaces and applications, Sci. China Math. 56 (2013), 831-844.

[38] Y. Jiao, D. Zhou, Z. Hao and W. Chen, Martingale Hardy spaces with variable exponents, Banach J. Math. Anal. 10 (2016), 750-770.

[39] Y. Jiao, D. Zhou, F. Weisz and Z. Hao, Corrigendum: Fractional integral on martingale Hardy spaces with variable exponents, Fract. Calc. Appl. Anal. 20 (2017), 1051-1052.

[40] Y. Jiao, D. Zhou, F. Weisz and L. Wu, Variable martingale Hardy spaces and their applications in Fourier analysis, arXiv: 1809.07520.

[41] W. B. Johnson and G. Schechtman, Martingale inequalities in rearrangement invariant function spaces, Israel J. Math. 64 (1988), 267-275.

[42] N. Kazamaki, Changes of law, martingales and the conditioned square function, Tôhoku Math. J. (2) 31 (1979), 549-552.

[43] M. Kbiri Alaoui, T. Nabil and M. Altanji, On some new non-linear diffusion models for the image filtering, Appl. Anal. 93 (2014), 269-280.

[44] M. Kikuchi, On Doob's inequality and Burkholder's inequality in weak spaces, Collect. Math. 67 (2016), 461-483.

[45] M. Kikuchi, A relation between two kinds of norms for martingales, Math. Slovaca 65 (2015), 1165-1180.

[46] M. Kikuchi, On some martingale inequalities for mean oscillations in weak spaces, Ric. Mat. 64 (2015), 137-165.

[47] Y. Liang, J. Huang and D. Yang, New real-variable characterizations of Musielak-Orlicz Hardy spaces, J. Math. Anal. Appl. 395 (2012), 413-428. 
[48] Y. Liang and D. Yang, Intrinsic square function characterizations of Musielak-Orlicz Hardy spaces, Trans. Amer. Math. Soc. 367 (2015), 3225-3256.

[49] K. Liu, D. Zhou and Y. Jiao, Hardy-Lorentz spaces for B-valued martingales, J. Math. Anal. Appl. 450 (2017), 1401-1420.

[50] R. L. Long, Martingale Spaces and Inequalities, Peking University Press, Beijing, Braunschweig, 1993.

[51] B. Matejczyk and A. Wróblewska-Kamińska, Unsteady flows of heat-conducting nonNewtonian fluids in Musielak-Orlicz spaces, Nonlinearity 31 (2018), 701-727.

[52] T. Miyamoto, E. Nakai and G. Sadasue, Martingale Orlicz-Hardy spaces, Math. Nachr. 285 (2012), 670-686.

[53] J. Musielak, Orlicz Spaces and Modular Spaces, Lecture Notes in Mathematics 1034, Springer-Verlag, Berlin, 1983.

[54] J. Musielak and W. Orlicz, On modular spaces, Studia Math. 18 (1959), 49-65.

[55] E. Nakai, G. Sadasue and Y. Sawano, Martingale Morrey-Hardy and Campanato-Hardy spaces, J. Funct. Spaces Appl. 2013, Art. ID 690258, 14 pp.

[56] E. Nakai and G. Sadasue, Maximal function on generalized martingale Lebesgue spaces with variable exponent, Statist. Probab. Lett. 83 (2013), 2168-2171.

[57] E. Nakai and G. Sadasue, Some new properties concerning BLO martingales, Tohoku Math. J. (2) 69 (2017), 183-194.

[58] H. Nakano, Modulared Semi-Ordered Linear Spaces, Maruzen Co., Ltd., Tokyo, 1950.

[59] A. Osȩkowski, Sharp $L^{p}$-bounds for the martingale maximal function, Tohoku Math. J. (2) 70 (2018), 121-138.

[60] A. Osȩkowski, Weighted maximal inequalities for the Haar system, Monatsh. Math. 186 (2018), 321-336.

[61] A. Osȩkowski, Inequalities for Hilbert operator and its extensions: the probabilistic approach, Ann. Probab. 45 (2017), 535-563.

[62] A. Osȩkowski, Weighted inequalities for martingale transforms and stochastic integrals, Mathematika 63 (2017), 433-450.

[63] A. Osȩkowski, Sharp Martingale and Semimartingale Inequalities, Mathematical Monographs (New Series) 72, Birkhäuser/Springer Basel AG, Basel, 2012.

[64] G. Pisier, Martingales in Banach Spaces, Cambridge Studies in Advanced Mathematics 155, Cambridge University Press, Cambridge, 2016.

[65] M. M. Rao and Z. D. Ren, Theory of Orlicz Spaces, Monographs and Textbooks in Pure and Applied Mathematics 146, Marcel Dekker, Inc., New York, 1991.

[66] G. Sadasue, Martingale Besov spaces and martingale Triebel-Lizorkin spaces, Sci. Math. Jpn. 2018 (to appear).

[67] F. Schipp and P. Simon, On some $\left(L^{1}, H\right)$-type maximal inequalities with respect to the Walsh-Paley system, in: Functions, Series, Operators, Vol. I, II (Budapest, 1980), 10391045, Colloq. Math. Soc. János Bolyai 35, North-Holland, Amsterdam, 1983.

[68] F. Schipp, W. R. Wade, P. Simon, and J. Pál, Walsh Series. An Introduction to Dyadic Harmonic Analysis, Adam Hilger, Bristol, New York, 1990.

[69] P. Simon, Cesàro summability with respect to two-parameter Walsh systems, Monatsh. Math. 131 (2000), 321-334. 
[70] P. Simon and F. Weisz, Weak inequalities for Cesàro and Riesz summability of WalshFourier series, J. Approx. Theory 151 (2008), 1-19.

[71] E. M. Stein, Topics in Harmonic Analysis Related to the Littlewood-Paley Theory, Annals of Mathematics Studies 63, Princeton University Press, Princeton, N.J.; University of Tokyo Press, Tokyo, 1970.

[72] J.-O. Strömberg and A. Torchinsky, Weighted Hardy Spaces, Lecture Notes in Mathematics 1381, Springer-Verlag, Berlin, 1989.

[73] A. S̀wierczewska-Gwiazda, Nonlinear parabolic problems in Musielak-Orlicz spaces, Nonlinear Anal. 98 (2014), 48-65.

[74] F. Weisz, Cesàro summability of one- and two-dimensional Walsh-Fourier series, Anal. Math. 22 (1996), 229-242.

[75] F. Weisz, Dual spaces of multi-parameter martingale Hardy spaces, Q. J. Math. 67 (2016), 137-145.

[76] F. Weisz, Martingale Hardy spaces for $0<p \leq 1$, Probab. Theory Related Fields 84 (1990), 361-376.

[77] F. Weisz, Martingale Hardy Spaces and Their Applications in Fourier Analysis, Lecture Notes in Mathematics 1568, Springer-Verlag, Berlin, 1994.

[78] F. Weisz, Summability of Multi-dimensional Fourier Series and Hardy Spaces, Mathematics and its Applications 541, Kluwer Academic Publishers, Dordrecht, 2002.

[79] G. Xie, Y. Jiao and D. Yang, Martingale Musielak-Orlicz Hardy spaces, Sci. China Math. (2018), DOI: 10.1007/s11425-000-0000-0.

[80] D. Yang, Y. Liang and L. D. Ky, Real-variable Theory of Musielak-Orlicz Hardy Spaces, Lecture Notes in Mathematics 2182, Springer, Cham, 2017.

[81] D. Yang, W. Yuan and C. Zhuo, Musielak-Orlicz Besov-type and Triebel-Lizorkin-type spaces, Rev. Mat. Complut. 27 (2014), 93-157.

Guangheng Xie and Dachun Yang (Corresponding author)

Laboratory of Mathematics and Complex Systems (Ministry of Education of China), School of Mathematical Sciences, Beijing Normal University, Beijing 100875, People's Republic of China

E-mails: guanghengxie@mail . bnu.edu.cn (G. Xie)

dcyang@bnu.edu.cn (D. Yang)

Ferenc Weisz

Department of Numerical Analysis, Eötvös L. University, H-1117 Budapest, Pázmány P. sétány 1/C., Hungary

E-mail: weisz@inf.elte.hu

Yong Jiao

School of Mathematics and Statistics, Central South University, Changsha 410075, People's Republic of China

E-mail: jiaoyong@csu.edu.cn 